\title{
Classification of the Acanthocephala
}

\author{
Omar M. Amin
}

Institute of Parasitic Diseases, Scottsdale, Arizona, USA

\begin{abstract}
In 1985, Amin presented a new system for the classification of the Acanthocephala in Crompton and Nickol's (1985) book 'Biology of the Acanthocephala' and recognized the concepts of Meyer (1931, 1932, 1933) and Van Cleave (1936, 1941, $1947,1948,1949,1951,1952)$. This system became the standard for the taxonomy of this group and remains so to date. Many changes have taken place and many new genera and species, as well as higher taxa, have been described since. An updated version of the 1985 scheme incorporating new concepts in molecular taxonomy, gene sequencing and phylogenetic studies is presented. The hierarchy has undergone a total face lift with Amin's (1987) addition of a new class, Polyacanthocephala (and a new order and family) to remove inconsistencies in the class Palaeacanthocephala. Amin and Ha (2008) added a third order (and a new family) to the Palaeacanthocephala, Heteramorphida, which combines features from the palaeacanthocephalan families Polymorphidae and Heteracanthocephalidae. Other families and subfamilies have been added but some have been eliminated, e.g. the three subfamilies of Arythmacanthidae: Arhythmacanthinae Yamaguti, 1935; Neoacanthocephaloidinae Golvan, 1960; and Paracanthocephaloidinae Golvan, 1969. Amin (1985) listed 22 families, 122 genera and 903 species (4, 4 and 14 families; 13, 28 and 81 genera; 167, 167 and 569 species in Archiacanthocephala, Eoacanthocephala and Palaeacanthocephala, respectively). The number of taxa listed in the present treatment is 26 families (18\% increase), 157 genera (29\%), and 1298 species (44\%) (4, 4 and 16; 18, 29 and 106; 189,255 and 845 , in the same order), which also includes 1 family, 1 genus and 4 species in the class Polyacanthocephala Amin, 1987, and 3 genera and 5 species in the fossil family Zhijinitidae.
\end{abstract}

Keywords: spiny-headed worms, species list, taxonomic system, Archiacanthocephala, Eoacanthocephala, Palaeacanthocephala, Polyacanthocephala

The present treatment is an updated version of the Amin (1985) system for the classification of the Acanthocephala, which has become the standard for the taxonomy of this group since its original publication in 1985 . That work was preceded by a synopsis and a classification scheme of the phylum (Amin 1982). This updated treatment includes hierarchal changes, a considerable number of new taxa, continues to retain its affiliation with the systems of Meyer $(1931,1932,1933)$ and Van Cleave (1936, 1941, 1947, 1948, 1949, 1951, 1952), and incorporates new contributions of molecular taxonomy and phylogenetic studies to the taxonomic system.

Amin (1985) included a detailed historical account of the Acanthocephala since the first recognizable reference to worms having proboscides was made by Redi (1684). Rudolphi (1802) was the first to name these worms Acanthocephala and gave them an ordinal rank with one genus, Echinorhynchus. Most early taxonomic descriptions lacked detailed morphological information until Lühe's (1904, 1905) critical review of the early descriptions.

Hamann (1892) recognized the diversity of this group of worms and fragmented the old genus Echinorhynchus into three families (Echinorhynchidae, Gigantorhynchidae, Neorhynchidae), which formed the basis of the more recent classification of the Acanthocephala. These conceptual divisions were elevated to the ordinal rank by Meyer (1931) and Van Cleave (1936), but to the subordinal rank by Southwell and MacFie (1925), among other variations by other observers including Travassos (1926), Thapar (1927), Witenberg (1932a,b), and Meyer (1931, 1932, 1933), among others.

The uncertainty about the position of the Acanthocephala among other animal groups was marked by Leuckart's (1848) speculation of lines of decent between the cestodes and the Acanthocephala, which he placed as two orders in his class Anenteraeti, and by Meyer (1932, 1933), who regarded the Acanthocephala as a class of the Aschelminthes including two orders, Palaeacanthocephala and Archiacanthocephala, on the basis of morphology and ontogeny.

Van Cleave (1936) removed the inconsistencies of Meyer's system by establishing a third order: Eoacanthocephala. He (Van Cleave 1941, 1948) recognized the Acanthocephala as a phylum closely associated with the Cestoda. More recently, Petrochenko (1956) devised a system based heavily on acanthor spination and Golvan (1959, 1960, 1961, 1969) considered the Eoacanthocephala (by now regarded as the most ancient group), Palaea-

Address for correspondence: O.M. Amin, Institute of Parasitic Diseases 11445 E. Via Linda, 2-419 Scottsdale, Arizona 85259, USA.

Phone: +480 767 2522; Fax:+480 767 5855; E-mail: omaramin@aol.com 
canthocephala and Archiacanthocephala to be classes, but relied heavily on the number of cement glands and trunk spination. Yamaguti (1963) recognized the three orders of Neoechinorhynchidea, Echinorhynchidea and Gigantorhynchidea corresponding to the Meyer-Van Cleave classes as well as a new fourth order: the Apororhynchidea. Golvan's (1994) nomenclature of the Acanthocephala culminated his life-long contributions to the systematics of the phylum.

Some of the more recent regional contributions to acanthocephalan taxonomy include those by Amin (2000), Salgado-Maldonado (2006), Bhattacharya (2007), and Salgado-Maldonado and Amin (2009). Amin (2000) listed and annotated the Acanthocephala in the Neotropical region. In an internal obscure publication, Bhattacharya (2007) listed 251 acanthocephalan species from India and described a few species and genera, but did not recognize order Neoechinorhynchida and included its families under order Gyracanthocephala. Salgado-Maldonado (2006) discussed and listed all helminth parasites of freshwater fishes in Mexico. Salgado-Maldonado and Amin (2009) discussed and listed the acanthocephalan species of the Gulf of Mexico.

Khatoon and Bilqees (1991) reviewed the classification of the Acanthocephala and included their version of Amin's (1985) historical introduction, a conventional taxonomy section of the higher taxa, and diagrammatic drawings of un-named representatives of discussed families. The taxonomic section included a number of misplacements of taxa without any justification. For example, among the higher taxa, they assigned order Polymorphida to class Polyacanthocephala and placed the families Pomphorhynchidae and Rhadinorhynchidae in order Polyacanthorhynchida. Later on, Khatoon and Bilqees (2011) published an expanded, well referenced version of their 1991 work updated through 1998 that included a list of species. The earlier (1991) assignments were not corrected and the species listing is outdated, incomplete and inaccurate in places that are beyond review at this time.

Amin (1982, 1985) recognized three classes: the Archiacanthocephala with four orders (Apororhynchida, Gigantorhynchida, Oligacanthorhynchida and Moniliformida), each with a single family, the Eoacanthocephala with two orders (Gyracanthocephala with one family and Neoechinorhynchida with three families), and the Palaeacanthocephala with two orders (Echinorhynchida with 11 families and Polymorphida with three families). This remained the accepted classification scheme until Amin (1987) added a fourth class to the phylum: Polyacanthocephala (and a new order and family) to remove inconsistencies in the class Palaeacanthocephala, and Amin and $\mathrm{Ha}$ (2008) added a third order (and a new family) to the Palaeacanthocephala: Heteramorphida, which combines features from the palaeacanthocephalan families Polymorphidae and Heteracanthocephalidae.
The number of lower taxa and families has also undergone marked increase since 1985, but some higher taxa have been eliminated, e.g. the three subfamilies of Arhythmacanthidae: Arhythmacanthinae Yamaguti, 1935, Neoacanthocephaloidinae Golvan, 1960, and Paracanthocephaloidinae Golvan, 1969 - see Amin et al. (2011a). Amin (1985) listed 22 families, 122 genera and 903 species (4, 4 and 14 families; 13, 28 and 81 genera; 167, 167 and 569 species in Archiacanthocephala, Eoacanthocephala, and Palaeacanthocephala, respectively). Monks and Richardson (2011) counted 4 classes, 10 orders, 22 families, 147 genera and 1194 species in the Acanthocephala as of December, 2011 and indicated that the number of species quoted is vastly underestimated.

The number of taxa listed in the present treatment is 26 families (18\% increase), 157 genera $(29 \%)$ and 1298 species $(44 \%)(4,4,16,1,1 ; 18,29,106,1,3 ; 189,255,845$, 4, 5 in the Archiacanthocephala, Eoacanthocephala, Palaeacanthocephala, Polyacanthocephala Amin, 1987, and the fossil family Zhijinitidae, respectively). These numbers do not include species listed in Appendix II (see p. 298-299).

Meyer $(1932,1933)$ grouped the Acanthocephala with the Rotifera, Gastrotricha, Kinorhyncha, Priapuloidea, Nematomorpha and Nematoda under the Aschelminthes. Recent molecular studies by Garey et al. (1996), GarcíaVarela et al. (2000), Welch (2000) and Near (2002), among others even suggest that Rotifera and Acanthocephala are phylogenetically related sister groups. Garey et al. (1996) and others, suggested that the Acanthocephala represent a taxon within phylum Rotifera. Several workers have since demonstrated the sister group relationship of Acanthocephala with Rotifera forming the phylum Syndermata Ahlrichs, 1997, e.g. Garey et al. (1998), Zrzavý (2001), García-Varela and Nadler (2006), Witek et al. (2008), Fontaneto and Jondelius (2011). Kristensen (2002) associated the Gnathostomulida with Micrognathozoa and the Syndermata into a larger clade called Gnathifera. The Gnathifera was first proposed by Rieger and Tyler (1995) and shown to be a monophyletic clade (Syndermata + Gnathostomulida) by Witek et al. (2009).

The phylogeny within the Syndermata subtaxon Acanthocephala was studied by sequencing the mitochondrial genomes of representatives of Palaeacanthocephala, Eoacanthocephala, Archiacanthocephala and Bdelloidea as well as of other syndermatans and 18 lophotrochozoan (spiralian) taxa, and one outgroup representative (Weber et al. 2013).

Phylogenetic analyses have shown that the monophyletic Archiacanthocephala represented the sister taxon of a clade comprising Eoacanthocephala and the monophyletic Palaeacanthocephala. This topology suggests the secondary loss of lateral sensory organs (sensory pores) in Palaeacanthocephala and is further in agreement with the emergence of apical sensory organs in the stem lineage of Archiacanthocephala (Weber et al. 2013). 
Because of the absence of gut in acanthocephalans and tapeworms, both groups have also been considered related. Cholodkovsky (1897) was the first to propose such a relationship since Leuckart's (1848) early accounts; this view was supported by Skrjabin and Shults (1931), Petrochenko (1952), Van Cleave (1941) and Amin et al. (2009). Structures interpreted as microtriches on the trunk epidermis of Rhadinorhynchus ornatus Van Cleave, 1918 (Rhadinorhynchidae) from skipjack tuna, Katsuwonus pelamis (Linnaeus), in the Pacific Ocean off South America were reported by Amin et al. (2009). Another marine rhadinorhynchid acanthocephalan, Leptorhynchoides polycristatus Amin, Heckmann, Halajian et El-Naggar, 2013, from sturgeons in the Caspian Sea appears to have similar structures. However, these structures do not appear to be homologous with microtriches of cestodes (see Chervy 2009 for details on microtriches in cestodes).

According to Garey et al. (1998), combining molecular and morphological analyses of Bilateria leads to a tree with Platyhelminthes, Rotifera, Acanthocephala and Gnathostomulida (and probably Gastrotricha) as a sister group to the annelid-mollusk lineage of the Spiralia (Lophotrochozoa). Steinauer et al. (2005), using mitochondrial (mt) genome sequences, suggested that Acanthocephala, as inferred from the $\mathrm{mt}$ genome of Leptorhynchoides the- catus (Linton, 1891), are closer to Platyhelminthes than was previously supposed. Their data are consistent with the data contained in numerous related studies based on RNA analysis. For instance, Min and Park (2009) linked the Syndermata with the Platyhelminthes as the Platyzoa.

Under all proposals, the monophyly of the major taxonomic groups of the Acanthocephala has been established (Near et al. 1998, Monks 2001, Near 2002) suggesting that the present classification of higher taxa is natural.

The following classification incorporates developments in phylogenetic and molecular taxonomy. Many taxonomic decisions based on molecular and gene sequence studies are made and incorporated in the text where they apply. Only valid generic and specific names are in bold, whereas invalid species are not listed. Species that have been relegated to other genera are listed under the recipient genera as synonyms. References to authors of scientific names and synonymies are not included. Fossil acanthocephalan taxa are listed in Appendix I. Genera incertae sedis assigned to Echinorhynchus sensu lato are listed in Appendix II. The indices of families and genera are listed after References, at the end of the article, to facilitate searching for individual taxa. Type species are listed within the alphabetical listing of the species and not separately at the beginning.

\section{CLASSIFICATION}

CLASS ARCHIACANTHOCEPHALA Meyer, 1931

(Monks 2001 did not support the Archiacanthocephala as a monophyletic group but García-Varela et al. 2000 authenticated the monophyly of the class as a sister group to the clade but García-Varela et al. 2000 authenticated the monophyly of the class as the basal class of the phylum and the sister group of a clade including Eoacanthocephala and Palaeacanthocephala.)

\section{ORDER APORORHYNCHIDA Thapar, 1927}

[syns. Apororhynchata Yokogawa et Morisita, 1933; Spheracanthocephala Byrd et Denton, 1949]

FAMILY Apororhynchidae Shipley, 1899

[syns. Arhynchidae Shipley, 1896; Arynchidae Monticelli, 1905]

GENUS Apororhynchus Shipley, 1899

[syns. Arhynchus Shipley, 1896; Neorhynchus de Marval, 1905]

\section{SPECIES}

A. aculeatus Meyer, 1931 (nec aculeatum)

A. amphistomi Byrd et Denton, 1949

A. bivolucrus Das, 1952 (nec bivoluerus)

(a strigeid trematode fide Yamaguti 1963)

A. chauhani Sen, 1975

A. hemignathi (Shipley, 1896) Shipley, 1899 (type species) (nec 1897)

[syns. Arhynchus hemignathi Shipley, 1896; Neorhynchus hemignathi (Shipley, 1896) de Marval, 1905]

A. paulonucleatus Khokhlova et Tsimbaluk, 1966 (nec 1971)

A. silesiacus Okulewicz et Maruszewski, 1980

ORDER GIGANTORHYNCHIDA Southwell et Macfie, 1925
FAMILY Gigantorhynchidae Hamann, 1892

[syn. Leiperacanthidae Bhalerao, 1937]

GENUS Gigantorhynchus Hamann, 1892

\section{SPECIES}

G. echinodiscus (Diesing, 1851) Hamann, 1892 (type species)

[syn. Echinorhynchus echinodiscus Diesing, 1851]

G. lopezneyrai Diaz-Ungria, 1958 (nec lopezneirae)

G. lutzi Machado Filho, 1941

G. ortizi Sarmiento, 1954

G. pesteri Tadros, 1966

G. ungriai Antonio, 1958 (nec ungariai)

GENUS Mediorhynchus Van Cleave, 1916

[syns. Disteganius Lehmann, 1953, nomen nudum; Empodisma Yamaguti, 1963; Empodius Travassos, 1916; Heteracanthorhynchus Lundström, 1942; Heteroplus Kostylew, 1914; Leiperacanthus Bhalerao, 1937; Micracanthorhynchus Travassos, 1917]

\section{SPECIES}

M. africanus Amin, Evans, Heckmann et El-Naggar, 2013

[syns. Empodius segmentatus (de Marval, 1902) Southwell et MacFie, 1925; Mediorhynchus selengensis, Harris, 1973; M. gallinarum (Bhalerao, 1937) Van Cleave, 1947 sensu Junker et Boomker, 2006] (Distinguishing $M$. africanus from the Asian M. gallinarum was based on morphological evidence, SEM and gene sequence analysis; Amin et al. 2013 used DNA sequence from one mitochondrial gene (cytochrome oxidase subunit 1) and one nuclear gene, 18S rRNA, to infer the phylogenetic relationships of $M$. africanus and M. gallinarum and selected Acanthocephala. Medioryhnchus was shown to be monophyletic and $M$. africanus and $M$. gallinarum to be allopatric sister species with $9.7 \%$ sequence divergence.)

M. alecturae (Johnston et Edmonds, 1947) Golvan, 1962 
M. cambellensis Soota, Srivastava et Glosh, 1969

M. centurorum Nickol, 1969

M. channapettae George et Nadakal, 1984

M. colluricinclae Smales, 2002

M. conirostris Ward, 1966

M. corcoracis Johnston et Edmonds, 1950

M. edmondsi Schmidt et Kuntz, 1977

M. emberizae (Rudolphi, 1819) Travassos, 1924

[syn. Echinorhynchus emberizae Rudolphi, 1819] (nec Van Cleave, 1916)

M. empodius (Skrjabin, 1913) Van Cleave, 1924 (nec Meyer, 1932) [syns. Gigantorhynchus empodius Skrjabin, 1913; Empodius empodius (Skrjabin, 1913) Travassos, 1916]

M. fatimaae Khan Bilqees et Muti-ur-Rahman, 2004

M. gallinarum (Bhalerao, 1937) Van Cleave, 1947

[syn. Leiperacanthus gallinarum Bhalerao, 1937]

(M. gallinarum is found only in Asia; 'Mediorhynchus gallinarum' in Africa is actually M. africanus Amin, Evans, Heckmann et ElNaggar, 2013)

M. gibsoni Bilqees, Khan, Khatoon et Khatoon, 2007

M. giganteus Meyer, 1931

[syns. Empodius giganteus (Meyer, 1931) Meyer, 1932; Empodisma giganteus (Meyer, 1931) Yamaguti, 1963] (nec giganteum)

M. grandis Van Cleave, 1916

[syn. Heteroplus grandis (Van Cleave, 1916) Van Cleave, 1918]

M. indicus George, Nadakal, Vijayakumaran et Rajendran, 1981

M. kuntzi Ward, 1960

M. lagodekhiensis Kuraschvili, 1955

M. lanius Amin, Ha et Heckmann, 2008

M. leptis Ward, 1966

M. lophurae Wang, 1966 (nec lophura)

M. mariae George et Nadakal, 1984

M. mattei Marchand et Vassiliades, 1982

M. meiringi Bisseru, 1960

M. micracanthus (Rudolphi, 1819) Meyer, 1932

[syns. Echinorhynchus alaudae Rudolphi, 1819; E. carrucioi Condorelli, 1897; E. micracanthus Rudolphi, 1819; Micracanthorhynchus micracanthus (Rudolphi, 1819) Travassos, 1917; Mediorhynchus armenicus Petrochenko, 1958]

M. mirabilis (de Marval, 1905) Travassos, 1924

[syn. Gigantorhynchus mirabilis de Marval, 1905]

M. muritensis Lundström, 1942

M. nickoli Khan, Bilqees et Muti-ur-Rahman, 2004

M. numidae (Baer, 1925) Meyer, 1932

[syns. Heteroplus numidae Baer, 1925; Empodisma numidae (Baer, 1925) Yamaguti, 1963]

M. orientalis Belopolskaya, 1953

[syn. Mediorhynchus bullocki Gupta et Jain, 1973]

M.oswaldocruzi Travassos, 1923

M. otidis (Miescher, 1841) Van Cleave, 1947

[syns. Echinorhynchus otidis Miescher, 1841; Heteroplus otidis (Miescher, 1841) Kostylew, 1914; Empodius otidis (Miescher, 1841) Travassos, 1917; Empodisma otidis (Miescher, 1841) Yamaguti, 1963]

M. pandei Bhattacharya, 2007

M. papillosus Van Cleave, 1916 (type species)

[syns. Empodius alecturae Johnston et Edmonds, 1947; Mediorhynchus bakeri Byrd et Kellogg, 1971; M. colini Webster, 1948]

M. passeris Das, 1951

M. pauciuncinatus Dollfus, 1959

M. peckeri Bhattacharya, 1999

M. peruensis Moya, Martinez et Tantalean, 2011

M. petrochenkoi Gvosdev et Soboleva, 1966

M. pintoi Travassos, 1923

M. quilonensis Bhattacharya, 2007

M. rajasthanensis Gupta, 1976

[M. najasthanensis Gupta, 1976, nomen nudum is a mis-spelling of M. rajasthanensis Gupta, 1976]

M. robustus Van Cleave, 1916

[syns. Mediorhynchus garruli Yamaguti, 1939 fide Schmidt and
Kuntz 1977 (synonymy termed 'questionable' by Golvan 1994); M. sipocotensis Tubangui, 1935]

M. rodensis Cosin, 1971

M. sipocotensis Tubangui, 1935 (nec sipposotensis, nec sipocotense)

M. spinaepaucites Smales, 2011

M. taeniatus (von Linstow, 1901) Dollfus, 1936

[syns. Echinorhynchus taeniatus von Linstow, 1901; E. segmentatus de Marval, 1902]

M. tenuis Meyer, 1931 (nec tenue)

M. textori Barus, Sixl et Majumdar, 1978

M. thrushi Bhattacharya, 2000

M. turdi Smales, 2011

M. turnixena (Tubangui, 1931) Webster 194

[syn. Empodius turnixena Tubangui, 1931]

M. vaginatus (Diesing, 1851) Meyer, 1932

[syn. Echinorhynchus vaginatus Diesing, 1851]

M. vancleavei (Lundström, 1942) Golvan, 1962

[syn. Heteracanthorhynchus vancleavei Lundström, 1942]

M. wardi Schmidt et Canaris, 1967

M. zosteropis (Porta, 1913) Meyer, 1932

[syn. Chentrorhynchus zosteropis Porta, 1913]

ORDER MONILIFORMIDA Schmidt, 1972

FAMILY Moniliformidae Van Cleave, 1924

GENUS Australiformis Schmidt et Edmonds, 1989

\section{SPECIES}

Australiformis semoni (von. Linstow, 1898) (type species)

[syns. Echinorhynchus semoni von Linstow, 1898; Moniliformis semoni (von Linstow, 1898) Johnston et Edmonds, 1952]

GENUS Moniliformis Travassos, 1915

[syns. Echinorhynchus Zoega in Müller, 1776, in part; Gigantorhynchus Hamann, 1892, in part; Hormorhynchus Ward, 1917]

\section{SPECIES}

M. acomysi Ward et Nelson, 1967

M. aegyptiacus Meyer, 1932

M. cestodiformis (von Linstow, 1904) Travassos 1917

[syns. Echinorhynchus cestodiformis von Linstow, 1904; Moniliformis erinacei Southwell et MacFie, 1925]

M. clarki (Ward, 1917) Chandler, 1921 (nec Van Cleave, 1924)

[syns. Hormorhynchus clarki Ward, 1917; Moniliformis spiradentatus MacLeod, 1933 (nec spiradentatis, nec spirodentatus) (fide Chandler 1941)]

M. convolutus Meyer, 1932

M. echinosorexi Deveaux, Schmidt et Krishnasamy, 1988

M. gracilis (Rudolphi, 1819) Meyer 1931

[syn. Echinorhynchus gracilis Rudolphi, 1819]

M. kalahariensis Meyer, 1931

M. merionis Golvan et Théodoridès, 1960

M. monechinus (von Linstow, 1902) Petrochenko, 1958

[syn. Echinorhynchus monechinus von Linstow, 1902]

M. moniliformis (Bremser, 1811) Travassos, 1915 (type species)

[syns. Echinorhynchus moniliformis Bremser, 1811; E. grassi Railliet, 1893; E. canis Porter, 1914; E. belgicus Railliet, 1919; Moniliformis moniliformis aegypticus Meyer in Petrochenko, 1958; M. dubius Meyer, 1932; M. travassosi Meyer, 1932 (fide Machado Filho 1946, Van Cleave 1952)]

M. monoechinus (von Linstow, 1902) Petrochenko, 1958 (nec monechinus)

M. myoxi (Galli-Valerio, 1929) (incertae sedis) [syn. Echinorhynchus myoxi Galli-Valerio, 1929]

M. siciliensis Meyer, 1932

[syns. M. m. siciliensis Meyer in Petrochenko, 1958; M. pseudosegmentatus (Knüppfer, 1888) Meyer, 1932: species inquirenda may be closely related if not identical to $M$. siciliensis, fide Golvan 1994)]

M. soricis (Rudolphi, 1819) (incertae sedis)

[syns. Echinorhynchus soricis Rudolphi, 1819; E. appendiculatus Westrumb, 1821] 
M. spiralis Subrahmanian, 1927

M. tarsii Deveaux, Schmidt et Krishnasamy, 1988

M. travassosi Meyer, 1932

GENUS Promoniliformis Dollfus et Golvan, 1963

SPECIES

Promoniliformis ovocristatus (von Linstow, 1897) Dollfus et

Golvan, 1963 (type species)

[syn. Echinorhynchus ovocristatus von Linstow, 1897]

ORDER OLIGACANTHORHYNCHIDA Petrochenko, 1956

FAMILY Oligacanthorhynchidae Southwell et Macfie, 1925

GENUS Cucullanorhynchus Amin, Ha et Heckmann, 2008

SPECIES

C. constrictruncatus Amin, Ha et Heckmann, 2008 (type species)

GENUS Heptamegacanthus Spencer-Jones, 1990

SPECIES

H. niekerki Spencer-Jones, 1990 (type species)

GENUS Macracanthorhynchus Travassos, 1917

[syns. Echinorhynchus Zoega in Müller, 1776, in part; Gigantorhynchus Hamann, 1892, in part]

SPECIES

M. catulinus Kostylew, 1927

M. erinacei Dollfus, 1953

M. hirudinaceus (Pallas, 1781) Travassos, 1917 (type species)

[syns. Taenia haeruca Pallas, 1776; T. hirudinacea Pallas, 1781; Echinorhynchus gigas (Bloch, 1782) Johnston, 1918; E. hirudinaceus (Pallas, 1781); Gigantorhynchus gigas Bloch, 1782; G. hirudinaceus (Pallas, 1781) Hamann, 1892; Hormorhynchus gigas (Bloch, 1782) Johnston, 1918; H. hirudinaceus Johnston, 1918]

M. ingens (von Linstow, 1879) Meyer, 1932

[syns. Echinorhynchus hirudinaceus ingens von Linstow, 1879; Prosthenorchis ingens (von Linstow, 1879) Travassos, 1917]

GENUS Multisentis Smales, 1997

SPECIES

M. myrmecobius Smales, 1997 (type species)

GENUS Neoncicola Schmidt, 1972

SPECIES

N. artibei Smales, 2007

N. avicola (Travassos, 1917) Schmidt, 1972

[syn. Prosthorhynchus avicola Travassos, 1917]

N. bursata (Meyer, 1931) (type species)

[syn. Oncicola bursata Meyer, 1931]

N. curvata (von Linstow, 1897) Schmidt, 1972

[syns. Echinorhynchus curvatus von Linstow, 1897; Prosthenorchis curvatus (von Linstow, 1897) Travassos, 1917]

N. novellae (Parona, 1890) Schmidt, 1972

[syns. Echinorhynchus novellae Parona, 1890; Prosthenorchis novella (Parona, 1890) Travassos, 1917]

N. pintoi (Machado Filho, 1950) Schmidt, 1972

[syn. Prosthenorchis pintoi Machado Filho, 1950]

N. potosi (Machado Filho, 1950) Schmidt, 1972

[syn. Prosthenorchis potosi Machado Filho, 1950]

N. sinensis Schmidt et Dunn, 1974

N. skrjabini (Morosow, 1951) Schmidt, 1972

[syn. Oncicola skrjabini Morosow, 1951]

GENUS Nephridiacanthus Meyer, 1931

[syn. Nephridiorhynchus Meyer, 1931]

SPECIES

N. gerberi Baer, 1959

[syn. Oligacanthorhynchus gerbera (Baer, 1959) Schmidt, 1972]

N. kamerunensis Meyer, 1931 (type species)

[syn. Oligacanthorhynchus kamerunensis (Meyer, 1931) Schmidt, 1972]
N. longissimus Golvan, 1962

syn. Oligacanthorhynchus longissimus (Golvan, 1962) Schmidt, 1972]

N. major (Bremser, 1811) Golvan, 1962

[syns. Echinorhynchus major Bremser, 1811; Gigantorhynchus major (Bremser, 1811) Porta, 1908]

N. manisensis Meyer, 1931

[syn. Oligacanthorhynchus manisensis (Meyer, 1931) Schmidt, 1972]

N. maroccanus Dollfus, 1951

N. palawanensis (Tubangui et Masilungan, 1938) Golvan, 1962 [syn. Nephridiorhynchus palawanensis Tubangui et Masilungan, 1938]

N. thapari (Sen et Chauhan, 1972) Golvan, 1994

[syn. Nephridiorhynchus thapari Sen et Chauhan, 1972]

GENUS Oligacanthorhynchus Travassos, 1915

[syns. Echinorhynchus Zoega in Müller, 1776, in part; Gigantorhynchus Hamann, 1892, in part; Echinopardalis Travassos, 1918; Hamanniella Travassos, 1915; Pardalis Travassos, 1917; Travassosia Meyer, 1931]

\section{SPECIES}

O. aenigma (Reichensperger, 1922) Meyer, 1932

[syn. Echinorhynchus aenigma Reichensperger, 1922]

O. atratus (Meyer, 1931) Schmidt, 1972

[syn. Echinopardalis atrata Meyer, 1931]

O. bangalorensis (Pujatti, 1951) Schmidt, 1972

[syn. Echinopardalis bangalorensis Pujatti, 1951]

O. carinii (Travassos, 1917) Schmidt, 1972

[syn. Hamanniella carinii Travassos, 1917; Travassosia carinii (Travassos, 1917) Meyer, 1932]

O. cati (Gupta et Lata, 1967) Schmidt, 1972

[syn. Hamanniella cati Gupta et Lata, 1967]

O. circumflexus (Molin, 1858) Meyer, 1932

[syn. Echinorhynchus circumflexus Molin, 1858]

O. citilli (Rudolphi, 1806) Kostylew et Zmeev, 1939

[syn. Echinorhynchus citilli Rudolphi, 1806]

O. compressus (Rudolphi, 1802) Meyer, 1932

[syns. Echinorhynchus compressus Rudolphi, 1802; E. cornicis (Rudolphi, 1819); E. macracanthus de Marval, 1902; Gigantorhynchus compressus (Rudolphi, 1802) de Marval, 1905]

O. decrescens (Meyer, 1931) Schmidt, 1972

[syn. Echinopardalis decrescens Meyer, 1931]

O. erinacei (Rudolphi, 1793) Meyer, 1932

[syns. Echinorhynchus erinacei Rudolphi, 1793; E. napaeformis Rudolphi, 1802; E. mustelae Rudolphi, 1819; E. kerkoides Westrumb, 1821; Prosthorhynchus erinacei (Rudolphi, 1802) Stiles et Stanley, 1932]

O. hamatus (von Linstow, 1897) Schmidt, 1972

[syns. Echinorhynchus hamatus von Linstow, 1897; Gigantorhynchus hamatus (von Linstow, 1897) Porta, 1908; Nephridiacanthus hamatus (von Linstow, 1897) Meyer, 1932]

o. iheringi Travassos, 1917

[syn. Echinorhynchus lagenaeformis Diesing, 1851, in part]

O. indicus Rengaraju et Das, 1981

O. kamtschaticus Khokhlova, 1966

O. lagenaeformis (Westrumb, 1821) Travassos, 1917

[syns. Echinorhynchus lagenaeformis Westrumb, 1821; E. falconis cyanei Rudolphi, 1819]

O. lamasi (Freitas et Costa, 1964) Amato, Nickol et Froés, 1979

[syn. Echinopardalis lamasi Freitas et Costa, 1964]

O. lerouxi Bisseru, 1956

[syn. Echinopardalis lerouxi Bisseru, 1956]

O. macrurae Meyer, 1931

[syn. Echinopardalis macrurae Meyer, 1931]

O. major (Machado Filho, 1963) Schmidt, 1972

[syn. Macracanthorhynchus major Machado Filho, 1963]

O. manifestus (Leidy, 1851) Van Cleave, 1924

[syn. Echinorhynchus manifestus Leidy, 1851]

O. mariemilyi (Tadros, 1969) Amin, 1985

[syn. Echinopardalis mariemilyi Tadros, 1969]

O. microcephala (Rudolphi, 1819) Schmidt, 1972 
[syns. Echinorhynchus microcephala Rudolphi, 1819; Hamanniella microcephala (Rudolphi, 1819) Travassos, 1915]

o. minor Machado Filho, 1964

O. nickoli Bolette, 2007

O. oligacanthus (Rudolphi, 1819) Meyer, 1932

[syn. Echinorhynchus oligacanthus Rudolphi, 1819]

O. oti Machado Filho, 1964

O. pardalis (Westrumb, 1821) Schmidt, 1972

[syns. Echinorhynchus pardalis Westrumb, 1821; Pardalis pardalis (Westrumb, 1821) Travassos, 1917; Echinopardalis pardalis (Westrumb, 1821) Travassos, 1918]

o. ricinoides (Rudolphi, 1808) Meyer, 1931

[syns. Echinorhynchus ricinoides Rudolphi, 1808; E. charadriiplurialis (Rudolphi, 1819); E. coraciae Rudolphi in Westrumb, 1821;

E. macracanthus Bremser in Westrumb, 1821]

O. shillongensis (Sen et Chauhan, 1972) Amin, 1985

[syn. Nephridiacanthus shillongensis Sen et Chauhan, 1972]

O. spira (Diesing, 1851) Travassos, 1915 (type species)

[syns. Echinorhynchus spira Diesing, 1851; E. uromasticus Fraipoint,

1882; Gigantorhynchus aurae Travassos, 1912]

o. taenioides (Diesing, 1851) Travassos 1915

[syns. Echinorhynchus oligacanthoides Rudolphi, 1819, in part;

E. taenioides Diesing, 1851]

o. thumbi Haffner, 1939

O. tortuosa (Leidy, 1850) Schmidt, 1972

[syns. Echinorhynchus tortuosa Leidy, 1850; Hamanniella tortuosa (Leidy, 1850) Van Cleave, 1924]

O. tumida (Van Cleave, 1947) Schmidt, 1972

[syns. Travassosia tumida Van Cleave, 1947; Hamanniella tumida (Van Cleave, 1947) Van Cleave, 1953]

GENUS Oncicola Travassos, 1916

\section{SPECIES}

O. campanulata (Diesing, 1851) Meyer, 1931

[syns. Echinorhynchus campanulata Diesing, 1851; Echinorhynchus ovatus Leidy, 1850]

O. canis (Kaupp, 1909) Hall et Wigdor, 1918

[syn. Echinorhynchus canis Kaupp, 1909]

O. chibigouzouensis Machado Filho, 1963

o. confusa (Machado Filho, 1950) Schmidt, 1972

[syn. Prosthenorchis confusus Machado Filho, 1950] (nec confusus)]

O. dimorpha Meyer, 1931

O. freitasi (Machado Filho, 1950) Schmidt, 1972

[syn. Prosthenorchis freitasi Machado Filho, 1950]

o. gigas Meyer, 1931

O. juxtatesticularis (Machado Filho, 1950) Schmidt, 1972

[syn. Prosthenorchis justatesticularis Machado Filho, 1950]

O. luehei (Travassos, 1917) Schmidt, 1972

[syn. Prosthenorchis luehei Travassos, 1917]

O. machadoi Schmidt, 1972

[syn. Prosthenorchis travassosi Machado Filho, 1950]

O. macrurae Meyer, 1931

[syn. Echinopardalis macrurae (Meyer, 1931) Witenberg, 1938]

O. magalhaesi Machado Filho, 1962

O. malayanus Toumanoff, 1947

O. martini Schmidt, 1977

o. michaelseni Meyer, 1932

O. micracantha Machado Filho, 1949

O. oncicola (Ihering, 1892) Travassos, 1916 (type species) [syn. Echinorhynchus oncicola Ihering, 1892]

O. paracampanulata Machado Filho, 1963

O. pomatostomi (Johnston et Cleland, 1912) Schmidt, 1983

[syns. Echinorhynchus pomatostomi Johnston et Cleland, 1912; Oligacanthorhynchus pomatostomi (Johnston et Cleland, 1912) Tubangui, 1933]

O. schacheri Schmidt, 1972

O. sigmoides (Meyer, 1932) Schmidt, 1972

[syn. Prosthenorchis sigmoides Meyer, 1932]

O. spirula (Olfers in Rudolphi, 1819) Schmidt, 1972

[syns Echinorhynchus spirula Olfers in Rudolphi, 1819; Prosthenor- chis spirula (Olfers in Rudolphi, 1819) Travassos, 1917; P. elegans Travassos, 1917]

O. travassosi Witenberg, 1938

O. venezuelensis Marteau, 1977

GENUS Pachysentis Meyer, 1931

SPECIES

P. angolensis (Golvan, 1957) Schmidt, 1972

[syn. Oncicola angolensis Golvan, 1957]

P. canicola Meyer, 1931 (type species) (fide Van Cleave 1953)

P. dollfusi (Machado Filho, 1950) Schmidt, 1972

[syn. Prosthenorchis dollfusi Machado Filho, 1950]

P. ehrenbergi Meyer, 1931

P. gethi (Machado Filho, 1950) Schmidt, 1972

[syn. Prosthenorchis gethi Machado Filho, 1950]

P. lenti (Machado Filho, 1950) Schmidt, 1972

[syn. Prosthenorchis lenti Machado Filho, 1950]

P. procumbens Meyer, 1931

P. procyonis (Machado Filho, 1950) Schmidt, 1972 [syn. Prosthenorchis procyonis Machado Filho, 1950]

P. rugosus (Machado Filho, 1950) Schmidt, 1972

[syn. Prosthenorchis rugosus Machado Filho, 1950]

P. septemserialis (Machado Filho, 1950) Schmidt, 1972

[syn. Prosthenorchis septemserialis Machado Filho, 1950]

GENUS Paraprosthenorchis Amin, Ha et Heckmann, 2008

SPECIES

P. ornatus Amin, Ha et Heckmann, 2008 (type species)

GENUS Prosthenorchis Travassos, 1915

SPECIES

P. elegans (Diesing, 1851) Travassos, 1915 (type species)

[syn. Echinorhynchus elegans Diesing, 1851]

P. fraterna (Baer, 1959) Schmidt, 1972

[syn. Oncicola fraterna Baer, 1959]

P. lemuri Machado Filho, 1950

[syn. P. elegans sensu Dollfus, 1938]

$\boldsymbol{P}$ pardalis Southwell et MacFie, 1925 (nomen nudum)

P. sinicus Hu-Jiand, 1990

GENUS Tchadorhynchus Troncy, 1970

SPECIES

T. quentini Troncy, 1970 (type species)

CLASS EOACANTHOCEPHALA Van Cleave, 1936

ORDER GYRACANTHOCEPHALA Van Cleave, 1936

FAMILY Quadrigyridae Van Cleave, 1920

[syns. Acanthogyridae Thapar, 1927; Pallisentidae Van Cleave, 1928]

SUBFAMILY Pallisentinae Van Cleave, 1928

GENUS Acanthogyrus Thapar, 1927

[syn. Hemigyrus Achmerov et Dombrovskaja, 1941]

SUBGENUS Acanthogyrus Thapar, 1927

SPECIES

A. (A.) acanthogyrus Thapar, 1927 (type species)

[syn. Acanthogyrus guptai Gupta et Verma, 1976] (fide Farooqi 1989)

A. (A.) tripathii Rai, 1967 (nec tripathi)

SUBGENUS Acanthosentis Verma et Datta, 1929

(Amin 2005 reviewed the subgenus and its zoogeography, identified problematic species, and provided a key to the valid species)

\section{SPECIES}

A. (A.) acanthuri (Cable et Quick, 1954) Amin, 1985

[Acanthosentis acanthuri Cable et Quick, 1954; A. acanthuri (Cable

et Quick, 1954) Golvan, 1959] 
A. (A.) adriaticus Amin, 2005

[syn. Acanthogyrus lizae Orecchia, Paggi et Radujkovic, 1988]

A. (A.) alternatspinus Amin, 2005

A. (A.) anguillae (Wang, 1981) Amin, 1985

[syn. Acanthosentis anguillae Wang, 1981]

A. (A.) antespinus (Verma et Datta, 1929) Amin, 1985 (type species)

(original spelling 'antspinus' was corrected by Meyer 1932; nec antespinis)

[syns. Acanthosentis antspinus Verma et Data, 1929; A. antspinus (Verma et Datta, 1929) Dollfus et Golvan, 1956; A. oligospinus Anantaraman, 1980]

A. (A.) arii (Bilqees, 1971) Amin, 1985

[syn. Acanthosentis arii Bilqees, 1971]

A (A.) bacailai (Verma, 1973) Amin, 1985

[syn. Acanthosentis bacailai Verma, 1973]

A. (A.) barmeshoori Amin, Gholami, Akhlaghi et Heckmann, 2013

A. (A.) betwai (Tripathi, 1959) (nec 1956) Amin, 2005

[syn. Acanthosentis betwai Tripathi, 1959]

A. (A.) bilaspurensis (Chowhan, Gupta et Khera, 1987) comb. n.

[syn. Acanthosentis bilaspurensis Chowhan, Gupta et Khera, 1987]

A. (A.) commersoni Gupta et Kajaji, 1969 (nec cammersoni)

[syn. Acanthosentis cameroni Amin, 1985]

A. (A.) cheni Amin, 2005

[syns. Acanthosentis coiliae (Yamaguti, 1939) sensu Chen, 1973;

Neoechinorhynchus coiliae Yamaguti, 1939].

A. (A.) dattai (Podder, 1938) (nec 1933) Amin, 1985

[syns. Acanthosentis dattai Podder, 1938; A. dattai (Podder, 1938)

Dollfus et Golvan, 1956]

A. (A.) giuris (Soota et Sen, 1956) Amin, 1985

[syn. Acanthosentis giuris Soota et Sen, 1956]

A. (A.) gobindi (Chowhan, Gupta et Khera, 1987) comb. n.

[syn. Acanthosentis gobindi Chowhan, Gupta et Khera, 1987]

A. (A.) golvani (Gupta et Jain, 1980) Amin, 1985

[syn. Acanthosentis golvani Gupta et Jain, 1980]

A. (A.) heterospinus (Khan et Bilqees, 1990) comb. n.

A. (A.) holospinus (Sen, 1937) Amin, 1985

[syn. Acanthosentis holospinus Sen, 1937; A. holospinus (Sen, 1937) Dollfus et Golvan, 1956]

A. (A.) indica (sic!) (Tripathi, 1959) Amin, 1985

[syns. Acanthosentis hilsai Pal, 1963; A. indicus Tripathi, 1959; A. indica (Tripathi, 1959) Chubb, 1982]

A. (A.) intermedius (Achmerov et Dombrowskaya-Achmerova, 1941) Amin, 1985

[syns. Hemigyrus intermedius Achmerov et Dombrowskaya-Achmerova, 1941; Acanthocephalorhynchoides intermedius (Achmerov et Dombrowskaya-Achmerova, 1941) Petrochenko, 1956]

A. (A.) lizae Wang, 1986

A. (A.) malawiensis Amin et Hendrix, 1999

A. (A.) maroccanus (Dollfus, 1951) Amin, 1985

[syns. Acanthosentis maroccanus Dollfus, 1951; A. maroccanus (Dollfus, 1951) Dollfus et Golvan 1956]

A. (A.) multispinus (Wang, 1966) Amin, 1985

[syn. Acanthosentis multispinus Wang, 1966]

A. (A.) nigeriensis (Dollfus et Golvan, 1956) Amin, 1985

[syn. Acanthosentis nigeriensis Dollfus et Golvan, 1956]

A. (A.) papilo (Troncy et Vassiliades, 1974) Amin, 1985

[syn. Acanthosentis papilo Troncy et Vassiliades, 1974]

A. (A.) parareceptaclis Amin, 2005

A. (A.) partispinus (Furtado, 1963) Amin, 1985 [syn. Acanthosentis partispinus Furtado, 1963]

A. (A.) paucispinus Wang, 1966

A. (A.) periophthalmi (Wang, 1980) Amin, 1985

[syn. Acanthosentis periophthalmi Wang, 1980]

A. (A.) phillipi (Mashego, 1988) Amin, 2005

[syn. Acanthosentis phillipi Mashego, 1988]

A. (A.) putitorae (Chowhan, Gupta et Khera, 1988) Amin, 2005

[syn. Acanthosentis putitorae Chowhan, Gupta et Khera, 1988]

A. (A.) scomberomori (Wang, 1980) Amin, 1985

[syn. Acanthosentis scomberomori Wang, 1980]

A. (A.) seenghalae (Chowhan, Gupta et Khera, 1988) Amin, 2005

[syn. Acanthosentis seenghalae Chowhan, Gupta et Khera, 1988]
A. (A.) shashiensis (Tso, Chen et Chien, 1974) Amin, 1985 [syn. Acanthosentis shashiensis Tso, Chen et Chien, 1974]

A. (A.) shuklai (Agrawal et Singh, 1982) Amin, 2005 [syn. Acanthosentis shuklai Agrawal et Singh, 1982]

A. (A.) siamensis (Farooqi et Sirikanchana, 1987) Amin, 2005 [syn. Acanthosentis siamensis Farooqi et Sirikanchana, 1987]

A. (A.) similis (Wang, 1980) Amin, 1985

[syn. Acanthogyrus (Acanthosentis) sp. of Wang 1966; Acanthosentis similis Wang, 1980]

A. (A.) sircari (Podder, 1941) Amin, 1985

[syn. Acanthosentis sircari Podder, 1941; A. sircari (Podder, 1941) Dollfus et Golvan, 1956]

A. (A.) thapari (Parasad, Sahay et Shambhunath, 1969) Amin, 1985 [syn. Acanthosentis thapari Parasad, Sahay et Shambhunath, 1969]

A. (A.) tilapiae (Baylis, 1948) Amin, 1985

[syn. Acanthosentis tilapiae Baylis, 1948]

A. (A.) tripathi Rai, 1967

A. (A.) vancleavei (Gupta et Fatma, 1986) Amin, 2005

[syn. Acanthosentis vancleavei Gupta et Fatma, 1986]

A. (A.) vittatusi (Verma, 1973) Amin, 1985

[syn. Acanthosentis vittatusi Verma, 1973]

GENUS Palliolisentis Machado Filho, 1960

\section{SPECIES}

P. ornatus Machado Filho, 1960

P. polyonca Schmidt et Hugghins, 1973

P. quinqueungulis Machado Filho, 1960 (type species)

GENUS Pallisentis Van Cleave, 1928

[syns. Devendrosentis Sahay, Sinha et Ghosh, 1971; Farzandia Thapar, 1931; Neosentis Van Cleave 1928; Saccosentis Tadros, 1966]

(Revision by Amin et al. 2000 created three subgenera based on the size of proboscis hooks.)

SUBGENUS Brevitritospinus Amin, Heckmann, Ha, Luc et Doanh, 2000

(posterior hooks about half as long as middle hooks)

SPECIES

P. (B.) allahabadi Agarwal, 1958 (type species)

[syn. P. buckleyi Tadros, 1966] (nec allahabadii)

P. (B.) cavasii Gupta et Verma, 1980

P. (B.) croftoni Mital et Lal, 1981

P. (B.) fasciati Gupta et Verma, 1980 (nec fasciata)

P. (B.) fotedari Gupta et Sinha, 1991

P. (B.) guntei Sahay, Nath et Sinha, 1967

P. (B.) indica Mital et Lal, 1981

P. (B.) jagani Koul, Raina, Bambroo et Koul, 1991

P. (B.) mehrai Gupta et Fatma, 1986

P. (B.) vietnamensis Amin, Heckmann, Ha, Luc et Doanh, 2000 [syn. Pallisentis ophiocephali Moravec et Sey, 1989]

(The identification of $P$. vietnamensis $\mathrm{sp}$. $\mathrm{n}$. as $P$. ophiocephali by Moravec and Sey 1989 overlooked the difference in proboscis hook size, these species belong in two different subgenera, and the fact that trunk spines of the latter species extend to the posterior ends of individuals of both sexes Amin et al. 2000.)

SUBGENUS Demidueterospinus Amin, Heckmann, Ha, Luc et Doanh, 2000

(middle hooks about half as long as anterior hooks)

SPECIES

P. (D.) basiri Farooqi, 1958

P. (D.) ophiocephalus (Thapar 1931) Baylis, 1933 (type species)

[syns. Farzandia ophiocephali Thapar, 1931; Pallisentis magnum Saeed et Bilqees, 1971] (nec ophiocephali)

P. (D.) panadei Raj, 1967

SUBGENUS Pallisentis Van Cleave, 1928 sensu stricto

(hooks gradually decrease in size posteriorly) 
SPECIES

P. (P.) celatus (Van Cleave, 1928) Baylis, 1933

[syn. Neosentis celatus Van Cleave, 1928] (nec cleatus)

P. (P.) cholodkowskyi (Kostylew, 1928) Amin, 1985

[syns. Quadrigyrus cholodkowskyi Kostylew, 1928; Acanthogyrus cholodkowskyi (Kostylew, 1928) Golvan, 1959; Acanthocephalynchoides cholodkowskyi (Kostylew, 1928) Williams, Gibson et Sadighian, 1980]

P. (P.) chongqingensis Liu et Zhang, 1993

P. (P.) colisai Sarkar, 1954

[syn. P. panadei Rai, 1967; P. buckley Tadros, 1966]

P. (P.) clupei Gupta et Gupta, 1980

P. (P.) gaboes (MacCallum, 1918) Van Cleave, 1928

[syns. Echinorhynchus gaboes MacCallum, 1918; Pallisentis (Farzandia) gaboes (MacCallum, 1918) Van Cleave, 1928]

P. (P.) garuai (Sahay, Sinha et Gosh, 1971) Jain et Gupta, 1979

(nec Gupta et Fatma, 1986)

[syn. Devendrosentis garuai Sahay, Sinha et Gosh, 1971]

P. (P.) gomtii Gupta et Verma, 1980

P. (P.) guptai Gupta et Fatma, 1986

P. (P.) jagani Koul, Raina, Bambroo et Koul, 1992

P. (P.) kalriai Khan et Bilqees, 1985

P. (P.) magnum Saeed et Bilqees, 1971

P. (P.) nagpurensis (Bhalerao, 1931) Baylis, 1931

[syn. Pallisentis (Farzandia) nagpurensis (Bhalerao, 1931) Baylis, 1933]

P. (P.) nandai Sarkar, 1953

P. (P.) pesteri (Tadros, 1966) Chowhan, Gupta et Khera, 1987

[syn. Saccosentis pesteri Tadros, 1966]

P. (P.) rexus Wongkham et Whitfield, 1999

P. (P.) sindensis Khan et Bilqees, 1987

P. (P.) umbellatus Van Cleave, 1928 (type species)

P. (P.) ussuriense (Kostylew, 1941) Golvan, 1959

[syn. Acanthocephalorhynchoides ussuriense Kostylew, 1941]

GENUS Pararaosentis Amin, Heckmann, Ha, Luc et Doanh, 2000

\section{SPECIES}

P. golvani (Troncy et Vassiliades, 1973) Amin, Heckmann, Ha, Luc et Doanh, 2000 (type species)

[syn. Pallisentis golvani Troncy et Vassiliades, 1973; Pallisentis tetraodontae Troncy, 1978]

(The characters on which Troncy 1978 based his assignment of

$P$. tetraodontae as a subspecies of $P$. golvani are not sufficient enough to justify a subspecific status, and $P$. tetraodontae is herein relegated to a synonym of $P$. golvani Amin et al. 2000.)

GENUS Raosentis Datta, 1947

SPECIES

R. dattai Gupta et Fatma, 1986

R. godavarensis Vankara et Vijayalakshmi, 2009

R. ivaniosi George et Nadakal, 1978

R. podderi Datta, 1947 (type species)

R. thapari Rai, 1967

GENUS Triaspiron Smales, Aydogdu et Emre, 2012

\section{SPECIES}

T. aphanii Smales, Aydogdu et Emre, 2012 (type species)

SUBFAMILY Quadrigyrinae Van Cleave, 1920

GENUS Acanthodelta Diaz-Ungria et Garcia-Rodrigo, 1958 (nec Acanthrodelta)

[syns. Deltacanthus Diaz-Ungria et Garcia-Rodrigo, 1958; Deltania Diaz-Ungria et Garcia-Rodrigo, 1957]

\section{SPECIES}

A. scorzai (Diaz-Ungria et Garcia-Rodrigo, 1957) Diaz-Ungria et Gracia-Rodrigo, 1958 (type species)

[syns. Deltania scorzai Diaz-Ungria et Garcia-Rodrigo, 1957; Deltacanthus scorzai (Diaz-Ungria et Garcia-Rodrigo, 1957) Diaz-Ungria et Gracia-Rodrigo, 1958]

GENUS Machadosentis Noronha, 1992

\section{SPECIES}

M. travassosi Noronha, 1992 (type species)

GENUS Quadrigyrus Van Cleave, 1920

\section{SPECIES}

Q. brasiliensis Machado Filho, 1941

Q. chinensis Mao, 1979

Q. guptai Gupta et Gunjan-Sinha, 1992

Q. machadoi Fabio, 1983

Q. nickoli Schmidt et Hugghins, 1973

Q. polyspinosus $\mathrm{Li}, 1984$

Q. rhodei Wang, 1980

Q. simhai Gupta et Fatma, 1986

Q. torquatus Van Cleave, 1920 (type species)

Q. torquatus sensu Ortlepp (1924)

(may be another species from Surinam fide Meyer 1932)

ORDER NEOECHINORHYNCHIDA Southwell et Macfie, 1925

[syn. Neoacanthocephala Van Cleave, 1936]

FAMILY Dendronucleatidae Sokolovskaya, 1962

GENUS Dendronucleata Sokolovskaya, 1962

SPECIES

D. americana Moravec et Huffman, 2000

D. dogieli Sokolovskaya, 1962 (type species)

D. petruschewskii Sokolovskaya, 1962

FAMILY Neoechinorhynchidae

(Ward, 1917) Van Cleave, 1928

[syns. Hebesomatidae Yamaguti, 1963; Hebesomidae Van Cleave, 1928; Neorhynchidae Hamann, 1892]

SUBFAMILY Atactorhynchinae Petrochenko, 1956

[syn. Floridosentinae Golvan, 1959]

GENUS Atactorhynchus Chandler, 1935

SPECIES

A. duranguensis Salgado-Maldonado, Aguilar-Aguilar et CabañasCarranza, 2005

A. verecundus Chandler, 1935 (type species)

GENUS Floridosentis Ward, 1953

(Rosas-Valdez et al. 2012 presented phylogenetic trees for two known species of Floridosentis, indicating that Floridosentis is monophyletic comprising of two major, well-supported clades corresponding with the two noted species and their geographical distribution.)

SPECIES

F. mugilis (Machado Filho, 1951) Bullock, 1962 (type species) [syns. Atactorhynchus mugilis Machado Filho, 1951; Floridosentis elongatus Ward, 1953]

F. pacifica Bravo-Hollis, 1969

GENUS Tanaorhamphus Ward, 1918

SPECIES

T. longirostris (Van Cleave, 1913) Ward, 1918 (type species)

[syns. Neorhynchus longirostris Van Cleave, 1913; Neoechinorhynchus longirostris (Van Cleave, 1913) Van Cleave, 1916]

SUBFAMILY Eocollinae Petrochenko, 1956

GENUS Eocollis Van Cleave, 1947

SPECIES

E. arcanus Van Cleave, 1947 (type species)

E. catostomi Buckner, 1992

E. harengulae Wang, 1981 
SUBFAMILY Gracilisentinae Petrochenko, 1956

GENUS Gracilisentis Van Cleave, 1919

\section{SPECIES}

G. gracilisentis (Van Cleave, 1913) Van Cleave, 1919 (type species) [syns. Neorhynchus gracilisentis Van Cleave, 1913; Neoechinorhynchus gracilisentis (Van Cleave, 1913) Van Cleave, 1916]

G. mugilus Gupta et Lata, 1967 (nec mugilis)

[syn. Gracilisentis mugilus sharmai Gupta et Lata, 1967]

G. sharmai Gupta et Lata, 1967

G. variabilis (Diesing, 1856) Petrochenko 1956

[syn. Echinorhynchus variabilis Diesing, 1856]

GENUS Pandosentis Van Cleave, 1920

\section{SPECIES}

P. iracundus Van Cleave, 1920 (type species)

P. napoensis Smales, 2007

GENUS Wolffhugelia Maňé-Garzon et Dei-Cas, 1974

SPECIES

W. matercula Maňé-Garzon et Dei-Cas, 1974 (type species)

SUBFAMILY Neoechinorhynchinae (Ward, 1917) Travassos, 1926

GENUS Dispiron Bilqees, 1970

SPECIES

D. catlai Khan et Bilqees, 1987

D. heteroacanthus Khan et Bilqees, 1985

D. mugilis Bilqees, 1970 (type species) (nec mugili)

GENUS Gorytocephalus Nickol et Thatcher, 1971

SPECIES

G. elongorchis Thatcher, 1979

G. plecostomorum Nickol et Thatcher, 1971 (type species)

G. spectabilis (Machado Filho, 1959) Nickol et Thatcher, 1971 [syn. Neoechinorhynchus spectabilis Machado Filho, 1959]

G. talaensis Vizcaino et Lunaschi, 1988

GENUS Hexaspiron Dollfus et Golvan, 1956

SPECIES

H. nigericum Dollfus et Golvan, 1956 (type species)

(nec nigeriensis)

H. spinibarbi Yu et Wang, 1977

GENUS Microsentis Martin et Multani, 1966

SPECIES

M. wardae Martin et Multani, 1966 (type species)

GENUS Neoechinorhynchus Stiles et Hassall, 1905

[syns. Echinorhynchus Zoega in Müller, 1776, in part; Eorhynchus Hamann, 1892; Neorhynchus Hamann, 1892; Eosentis Van Cleave, 1928]

(Revision by Amin 2002 created 2 subgenera based on egg structure, included a list of invalid and relegated species, and a key to 88 species.)

SUBGENUS Neoechinorhynchus Hamann, 1892

(eggs with concentric shells)

SPECIES

N. (N.) africanus Troncy, 1969

N. (N.) armenicus Mikailov, 1975

N. (N.) ascus Amin, Ha et Amin, 2011

N. (N.) australis Van Cleave, 1931 (nec australe)

N. (N.) beringianus Mikhailova et Atrashkevich, 2008

N. (N.) brentnickoli Monks, Pulido-Flores et Violante-Gonzalez, 2011

N. (N.) buckneri Amin et Heckmann, 2009
N. (N.) buttnerae Golvan, 1956

N. (N.) carassii Roytmann, 1961 (nec Rotman)

N. (N.) carpiodi Dechtiar, 1968

N. (N.) chelonos Schmidt, Esch et Gibbons, 1970

N. (N.) chilkaensis Podder, 1937

[syn. Neoechinorhynchus elongatus Tripathi, 1956] (fide Chandra et al. 1982)

N. (N.) chimalapasensis Salgado-Maldonado, 2010

N. (N.) crassus Van Cleave, 1919 (nec crassum)

N. (N.) cristatus Lynch, 1936 (nec cristatum)

N. (N.) curemai Noronha, 1973

N. (N.) cylindratus (Van Cleave, 1913) Van Cleave 1919

[syn. Neorhynchus cylindratus Van Cleave, 1913] (nec cylindratum)

N. (N.) dattai Golvan, 1994

[syn. Neoechinorhynchus rutili sensu Datta, 1936

(nec Müller, 1780)]

N. (N.) dimorphospinus Amin et Sey, 1996

N. (N.) distractus Van Cleave, 1949

[syn. in part Neoechinorhynchus australis Van Cleave, 1931] (nec distractum)

N. (N.) dorsovaginatus Amin et Christison, 2005

N. (N.) edmondsi Golvan, 1994

[syn. Neoechinorhynchus agilis sensu Edmonds, 1982]

N. (N.) emydis (Leidy, 1851) Van Cleave, 1919 (nec 1916)

[syns. Echinorhynchus emydis Leidy, 1851; E. hamulatus Leidy, 1856]

N. (N.) emyditoides Fisher, 1960

[syn. Neoechinorhynchus emydis sensu Bravo-Hollis, 1946] (nec Leidy, 1851)

N. (N.) formosanus (Harada, 1938) Kaw, 1951

[syn. Eosentis formosanus Harada, 1938]

(nec formosanum, nec formosans)

N. (N.) gibsoni Khan et Bilqees, 1989

(err: gilbesoni fide Golvan 1994)

N. (N.) golvani Salgado-Maldonado, 1978

(Salgado-Maldonado 2006 suggested the existence of two cryptic species of $N$. golvani, one associated with cichlids and the other with eleotrids in Mexico. Monks et al. 2011 subsequently described N. (N.) brentnickoli from eleotrid fishes. Martinez-Aquino et al. 2009 detected a complex of three cryptic species within $N$. golvani using two nuclear gene sequences that were associated with eleotrid and cichlid fish lineages in waters of different salinities.)

N. (N.) hartwichi Golvan, 1994

[syn. Neoechinorhynchus australis sensu Hartwich, 1956]

N. (N.) iraqensis Amin, Al-Sady, Mhaisen et Bassat, 2001

N. (N.) johnii Yamaguti, 1939 (nec johni)

N. (N.) limi Muzzall et Buckner, 1982

N. (N.) longnucleatus Amin, Ha et Ha, 2011

N. (N.) macronucleatus Machado Filho, 1954

(nec macronucleatum)

N. (N.) magnapapillatus Johnson, 1969

N. (N.) mamesi Pinacho-Pinacho, Pérez-Ponce de León et

García-Varela, 2012

(Species identity and distinction from $N$. brentnickoli and $N$. golvani were established by Pinacho-Pinacho et al. 2012, using morphology, genetic divergence with LSU and cox 1 sequences.)

N. (N.) moleri Barger, 2005

N. (N.) nawazi Naqvil, Aly Khan, Ghazi, et Noor-un-Nissa, 2012

N. (N.) nickoli Khan, Bilqees, Noor-Un-Nisa, Ghazi et Ata-Ur-

Rahim, 1999

N. (N.) notemigoni Dechtiar, 1967

N. (N.) panucensis Salgado Maldonado, 2013

N. (N.) paraguayensis Machado Filho, 1959

[syn. Echinorhynchus paucihamatum Leidy, 1890]

(nec paraguayense)

N. (N.) pimelodi Brasil-Sato et Pavanelli, 1998

N. (N.) plagiognathopitis Wang et Zhang, 1987

N. (N.) plaquensis Amin, Ha et Ha, 2011

N. (N.) prochilodorum Nickol et Thatcher, 1971

N. (N.) prolixoides Bullock, 1963 
N. (N.) prolixus Van Cleave et Timmons, 1952 (nec prolixum)

N. (N.) pseudemydis Cable et Hopp, 1954

[syn. Neoechinorhynchus constrictus Little et Hopkins, 1968

(Dezfuli and Tinti 1998 managed to separate specimens of N. pseudemydis from those of the $N$. emydis- $N$. emyditoides group using random-amplified polymorphic DNA (RAPD) analysis.)

N. (N.) pterodoridis Thatcher, 1981

N. (N.) qatarensis Amin, Saoud et Alkuwari, 2002

N. (N.) quinghaiensis Liu, Wang, et Yang, 1981 (nec 1980)

N. (N.) rigidus (Van Cleave, 1928) Kaw, 1951

[syn. Eosentis rigidus Van Cleave, 1928] (nec rigidum, nec rigidis)

N. (N.) robertbaueri Amin, 1985

N. (N.) roseum (sic!) Salgado-Maldonado, 1978 (emend.)

(nec Salgado et Maldonado)

N. (N.) rutili (Müller, 1780) (type species)

[syn. Echinorhynchus rutili Müller, 1780]

N. (N.) salmonis Ching, 1984

(Mikhailova 2013 recognized a polar population of $N$. salmonis from northern Asia different from the temperate population originally described from Canada in size, seasonality and developmental cycle.)

N. (N.) saurogobi Yi et Wu, 1989

N. (N.) schmidti Barger, Thatcher et Nickol, 2004

N. (N.) strigosus Van Cleave, 1949 (nec strigosum)

N. (N.) stunkardi Cable et Fisher, 1961 (vide Acholonu 1969)

N. (N.) sootai Bhattacharya, 1999

N. (N.) tenellus (Van Cleave, 1913) Van Cleave, 1919 [syn. Neorhynchus tenellus Van Cleave, 1913] (nec tenellum)

N. (N.) tumidus Van Cleave et Bangham, 1949 (nec tumidum)

N. (N.) tylosuri Yamaguti, 1939

[syn. Neoechinorhynchus asymetricus Belous, 1952]

N. (N.) venustus Lynch, 1936 (nec venustum)

N. (N.) villoldoi Vizcaino, 1992

N. (N.) wuyiensis Wang, 1981

N. (N.) zabensis Amin, Abdullah et Mhaisen, 2003

SUBGENUS Hebesoma Van Cleave, 1928

(eggs with polar prolongation of fertilization membrane)

\section{SPECIES}

N. (H.) agilis (Rudolphi, 1819) Van Cleave, 1916

[syn. Echinorhynchus agilis Rudolphi, 1819]

(Shih et al. 2010 differentiated between N. agilis, Neorhadinorhynchus macrospinosus and Rhadinorhynchus pristis using morphological, SEM and molecular methods. The nuclear ribosomal DNA region across the first internal transcribed spacer (ITS-1), the 5.8S gene and the second internal transcribed spacer (ITS-2) were amplified and the sizes of the PCR products were found to be different in length.)

N. (H.) anguillum El-Damarany, 2001

N. (H.) carinatus Buckner et Buckner, 1993

N. (H.) chrysemydis Cable et Hopp, 1954

N. (H.) didelphis Amin, 2001

N. (H.) doryphorus Van Cleave et Bangham, 1949 (nec doryphorum)

N. (H.) idahoensis Amin et Heckmann, 1992

N. (H.) kallarensis George et Nadakal, 1978

N. (H.) lingulatus Nickol et Ernst, 1987

N. (H.) manasbalensis Kaw, 1951 (nec manasbalense)

N. (H.) manubrianus Amin, Ha et Ha, 2011 (nec manubriensis)

N. (H.) pungitius Dechtiar, 1971

N. (H.) rostratus Amin et Bullock, 1998

N. (H.) violentus (Van Cleave, 1928) Salgado-Maldonado, 1978 (emend.) (type species) (nec violentum)

Valid species of Neoechinorhynchus not assigned to either subgenus; eggs unknown:

N. afghanus Moravec et Amin, 1978

N. ampullata Amin, Ha et $\mathrm{Ha}, 2011$

N. aldrichettae Edmonds, 1971

N. argentatus Chandra, Rao et Shyamasundari, 1984
N. bangoni Tripathi, 1956

N. brayi Bilqees, Shaikh et Khan, 2011

N. cirrhinae Gupta et Jain, 1979

N. coiliae Yamaguti, 1939

N. cyanophlyctic Kaw, 1951 (nec cyanophyctis)

N. devdevi (Datta 1936) Kaw, 1951

[syn. Eosentis yalei Datta, 1936; Neoechinorhynchus yalei (Datta,

1936) Kaw, 1951 fide Amin 2002]

N. glyptosternumi Fotedar et Dhar, 1977

(published as a new species by Dhar and Kharoo 1984)

N. hutchinsoni Datta, 1936

N. ichthyobori Saoud, El Naffar et Abu Sinna, 1974

N. indicus Gudivada, Chikkam et Vankara, 2010

N. karachiensis Bilqees, 1972

N. longilemniscus Yamaguti, 1954

N. longiorchis Khatoon et Bilqees, 2007

N. magnus Southwell et MacFee, 1925

N. nematalosi Tripathi, 1956

N. nigeriensis Farooqi, 1981

N. ningalooensis Pichelin et Cribb, 2001

N. octonucleatus Tubangui, 1933 (nec octonucleatum)

N. oreini Fotedar, 1968

N. ovalis Tripathi, 1956 (nec ovale)

N. roonwali Datta et Soota, 1961

N. saginatus Van Cleave et Bangham, 1949 (nec saginatum)

N. satori Morisita, 1937

N. simansularis Roitman, 1961

N. sinicus Wang, 1966

N. topseyi Podder, 1937

N. tsintaoensis Morisita, 1937 (nec tsintaoense)

N. zacconis Yamaguti, 1935

GENUS Octospinifer Van Cleave, 1919

SPECIES

O. macilentus Van Cleave, 1919 (type species)

o. rohitaii Zuberi et Farooq, 1976

O. torosus Van Cleave et Haderlie, 1950

O. variabilis (Diesing, 1851) Kritscher, 1976

[syn. Echinorhynchus variabilis Diesing, 1851]

GENUS Octospiniferoides Bullock, 1957

SPECIES

O. australis Schmidt et Hugghins, 1973

O. chandleri Bullock, 1957 (type species)

O. incognita Schmidt et Hugghins, 1973

GENUS Paraechinorhynchus Bilqees et Khan, 1983

SPECIES

P. kalriai Bilqees et Khan, 1983 (type species)

GENUS Paulisentis Van Cleave et Bangham, 1949

SPECIES

P. fractus Van Cleave et Bangham, 1949 (type species)

P. missouriensis Keppner, 1974

GENUS Zeylonechinorhynchus Fernando et Furtado, 1963

SPECIES

Z. longinuchalis Fernando et Furtado, 1963 (type species)

FAMILY Tenuisentidae Van Cleave, 1936

GENUS Paratenuisentis Bullock et Samuel, 1975

SPECIES

P. ambiguus (Van Cleave, 1921) Bullock et Samuel, 1975 (type species)

[syn. Tanaorhamphus ambiguus Van Cleave, 1921] 
(The proboscis of $P$. ambiguus appears to have an epidermal cone with three nuclei at the apex as per Herlyn 2001. Dendritic terminations, sensory nerves and secretory ducts were absent suggesting a mechanical function of the cone as have been suggested in other eoacanthocephalan reports. Herlyn 2001 proposed that the presence of epidermis cone only in the Eoacanthocephala supports its monophyly but draws no conclusions regarding the relationships with other acanthocephalan groups including polyacanthocephalans, e.g. $P$. kenyensis, with apical cones having demonstrable secretory ducts as described in Amin and Dezfuli 1995.)

\section{GENUS Tenuisentis Van Cleave, 1936}

SPECIES

T. niloticus (Meyer, 1932) Van Cleave, 1936 (type species)

[syn. Rhadinorhynchus niloticus Meyer, 1932]

\section{CLASS PALAEACANTHOCEPHALA Meyer, 1931}

(The criteria for the classification of families of Palaeacanthocephala based on morphological characteristics may need to be re-evaluated using gene sequence methods, see, e.g. García-Varela and Nadler 2005, to establish phylogenetic relationships. Verweyen et al. 2011 analyzed 39 species from all 4 classes of Acanthocephala using nuclear 18S rDNA sequences. They found that the resulting trees suggested a paraphyletic arrangement of the Echinorhynchida and Polymorphida inside the Palaeacanthocephala, which questions the placement of the genera Gorgorhynchoides and Serrasentis within the Echinorhynchida and not the Polymorphida.)

ORDER ECHINORHYNCHIDA Southwell et Macfie, 1925

\section{FAMILY Arhythmacanthidae Yamaguti, 1935}

(The three subfamilies, Arhythmacanthinae Yamaguti, 1935, Neoacanthocephaloidinae Golvan, 1960 and Paracanthocephaloidinae Golvan, 1969, as well as Yamagutisentinae Golvan, 1969 and Hypoechinorhynchidae Petrochenko, 1956 are unjustified and deleted, see Pichelin and Cribb 1999, Amin et al. 2011a.)

GENUS Acanthocephaloides Meyer, 1932

[syns. Neoacanthocephaloides Cable et Quick, 1954; Pseudorhynchus Petrochenko, 1956; Yamagutisentis Golvan, 1969]

SPECIES

A. claviformis Araki et Machida, 1987

A. cyrusi Bray, Spencer-Jones et Lewis, 1988

A. delamuri (Parukhin, 1989) comb. n.

[syn. Yamagutisentis delamuri Parukhin, 1989]

A. distinctus Golvan, 1969

A. geneticus de Buron, Renaud et Euzet, 1986

A. ichiharai Araki et Machida, 1987

A. incrassatus (Molin, 1858) Meyer, 1932

A. irregularis Amin, Oğuz, Heckmann, Tepe et Kvach, 2011

A. neobythitis (Yamaguti, 1939) comb. n.

[syns. Neoacanthocephaloides neobythitis Yamaguti 1939; Pseudorhadinorhynchus neobythitis Yamaguti, 1939; Yamagutisentis neobythitis (Yamaguti, 1939) Golvan, 1969]

A. nicoli (Kumar, 1992) comb. n.

[syn. Yamagutisentis nicoli Kumar, 1992]

A. plagiusae Piñeros, Quintana, Chalé et Martinez, 2013

A. propinquus (Dujardin, 1845) Meyer, 1932 (type species)

[syns. Echinorynchus propinquus Dujardin, 1845; E. fabri Rudolphi, 1819; E. kostylewi Meyer, 1932; E. pumilio Rudolphi, 1819] (nec propinguus)

A. rhinoplagusiae (Yamaguti, 1935) comb. n.

[syn. Yamagutisentis rhinoplagusiae (Yamaguti, 1935) Golvan, 1969] (nec rhynoplagusiae)

A. spinicaudatus (Cable et Quick, 1954) Pichelin et Cribb, 1999 [syn. Neoacanthocephaloides spinicaudatus Cable et Quick, 1954]

GENUS Bolborhynchoides Achmerov et Dombrovskaja, 1959

[syn. Bolborhynchus Achmerov et Dombrovskaja-Achmerova, 1941; Fresnyarhynchus Golvan, 1960]

SPECIES

B. exiguus (Achmerov et Dombrovskaja-Achmerova, 1941) Ach- merov 1959 (type species)

[syns. Bolborhynchus exiguus Achmerov et Dombrovskaja-Achmerova, 1941; Fresnyarhynchus exiguous (Achmerov et DombrovskajaAchmerova, 1941) Golvan, 1960]

GENUS Breizacanthus Golvan, 1969

\section{SPECIES}

B. aznari Hernández-Orts, Alama-Bermejo, Crespo, Garcia, Raga et Montero, 2012

B. chabaudei Golvan, 1969 (type species)

B. golvani Gaevskaya et Shukhgalter, 1984

B. irenae Golvan, 1969

B. ligur Paggi, Orecchia et Della Seta, 1975

GENUS Euzetacanthus Golvan et Houin, 1964

SPECIES

E. chorinemusi Gupta et Naqvi, 1984

E. golvani Gupta et Fatma, 1983

E. simplex (Rudolphi, 1810) Golvan et Houin 1964 (type species) [syns. Echinorynchus simplex Rudolphi, 1810; E. triglae gurnardi Rathike, 1799]

GENUS Heterosentis Van Cleave, 1931

[syn. Arhythmacanthus Yamaguti, 1935]

\section{SPECIES}

H. brasiliensis Vieira, Felizardo et Luque, 2009

H. fusiformis (Yamaguti, 1935) Tripathi, 1959

[syn. Arhythmacanthus fusiformis Yamaguti, 1935]

H. heteracanthus (von Linstow, 1896) Van Cleave, 1931

(type species)

[syn. Echinorhynchus heteracanthus von Linstow, 1896]

(nec hetracanthus)

H. hirsutus Pichelin et Cribb, 1999

H. holospinus Amin, Heckmann et Ha, 2011

H. martini Lanfranchi et Timi, 2011

H. mysturi Wei, Huang, Chen et Jiang, 2002

H. overstreeti (Schmidt et Paperna, 1978) Amin, 1985

[syn. Arhythmacanthus overstreeti Schmidt et Paperna, 1978]

H. paraplagusiarum (Nickol, 1972) Amin 1985

[syn. Arhythmacanthus paraplagusiarum Nickol, 1972]

H. parasiluri Yin et $\mathrm{Wu}, 1984$

H. plotosi (Yamaguti, 1935) Schmidt et Paperna, 1978

[syn. Arhythmacanthus plotosi Yamaguti, 1935]

H. pseudobagri (Wang et Zhang, 1987) Pichelin et Cribb, 1999

H. septacanthus (Sita, 1969) Amin, 1985

[syn. Arhythmacanthus septacanthus Sita in Golvan, 1969]

H. thapari (Gupta et Fatma, 1979) Amin, 1985

[syn. Arhythmacanthus thapari Gupta et Fatma, 1979]

H. zdzitowieckii (Kumar, 1992) Pichelin et Cribb, 1999

[syn. Arhythmacanthus zdzitowieckii Kumar, 1992]

GENUS Hypoechinorhynchus Yamaguti, 1939

(Hypoechinorhynchus was previously placed in family Hypoechinorhynchidae by Amin 1985.)

SPECIES

H. alaeopis Yamaguti, 1939 (type species)

H. golvani Gupta et Pramod-Kuma, 1987

H. magellanicus Szidat, 1950

H. robustus Pichelin, 1999

H. thermaceri de Buron, 1988

GENUS Paracanthocephaloides Golvan, 1969

\section{SPECIES}

P. cabelleroi (Gupta et Fatma, 1983) Bhattacharya, 2007 [syn. Heterosentis cabelleroi Gupta et Fatma, 1983]

P. chabanaudi (Dollfus, 1951) Golvan, 1969 (type species)

[syn. Acanthocephaloides chabanaudi Dollfus, 1951]

P. golvani Chandra, Hanumantha-Rao et Shyamasundari, 1984

P. incrassatus (Molin, 1858) Meyer, 1932 
[syns. Echinorynchus incrassatus Molin, 1858; E. devisiana Molin, 1858; E. flavus Molin, 1858; Acanthocephaloides incrassatus (Molin, 1858) Meyer, 1932 fide Bray et al. (1988)]

P. tripathii Golvan, 1969

[syn. Heterosentis plotosi (sensu Tripathi, 1959) Golvan, 1969]

GENUS Solearhynchus de Buron et Maillard, 1985

\section{SPECIES}

S. kostylewi (Meyer, 1932) Kvach et Oğuz, 2010

[syns. Paracanthocephaloides kostylewi Meyer, 1932; Acanthocephaloides kostylewi (Meyer, 1932) sensu Bray et al. 1988]

S. soleae (Porta, 1905) de Buron et Maillard, 1985 (type species)

[syns. Acanthocephaloides rhytidotes (Monticelli, 1904) Belofastova et Korniychus, 2000; Echinorynchus aurantiacus sensu Monticelli, 1887; E. corrogatus sensu Monticelli, 1887; E. rhytidotes Monticelli,

1905; E. soleae Porta, 1905; Acanthocephaloides soleae (Porta, 1905)

Meyer, 1932; Paracanthocephaloides soleae (Porta, 1905) Paggi et Orecchia, 1983; Solearhynchus rhytidotes (Monticelli, 1904) Belofastova, 2006]

GENUS Spiracanthus Muñoz et George-Nascimento, 2002

\section{SPECIES}

S. bovichthys Muñoz et George-Nascimento, 2002 (type species)

FAMILY Cavisomidae Meyer, 1932

[syn. Cavisomatidae Petrochenko, 1956]

GENUS Caballerorhynchus Salgado-Maldonado, 1977

SPECIES

C. lamothei Salgado-Maldonado, 1977 (type species)

GENUS Cavisoma Van Cleave, 1931

\section{SPECIES}

C. magnum (Southwell, 1927) Van Cleave, 1931 (type species) [syn. Oligoterorhynchus magnus Southwell, 1927]

GENUS Echinorhynchoides Achmerov et DombrovskajaAchmerova, 1941

\section{SPECIES}

E. dogieli Achmerov et Dombrovskaja-Achmerova, 1941 (type species)

[syn. Neorhadinorhynchus dogieli (Achmerov et DombrovskajaAchmerova, 1941) Yamaguti, 1963]

GENUS Femogibbosus Parukhin, 1973

SPECIES

F. assi Parukhin, 1973 (type species)

GENUS Filisoma Van Cleave, 1928

\section{SPECIES}

F. acanthocybii Wang, Wang et Wu, 1993

F. atropt Wang, 1988

F. bucerium Van Cleave, 1940 (nec bucerinum)

F. fidum Van Cleave et Manter, 1948

F. filiformis Weaver et Smales, 2013

F. indicum Van Cleave, 1928 (type species)

[syn. Filisoma hoogliensis Datta et Soota, 1962]

F. inglisi Gupta et Naqvi, 1984

F. longcementglandatus Amin et Nahhas, 1994

F. microcanthi Harada, 1938 (nec micracanthi, nec macrocanthi)

F. oplegnathi Wang, 1988

F. rizalinum Tubangui et Masilungan, 1946

F. scatophagusi Datta et Soota, 1962

GENUS Megapriapus Golvan, Garcia-Rodrigo et Diaz-Ungria, 1964

SPECIES

M. ungriai (Garcia-Rodrigo, 1960) (type species)

[syn. Echinorhynchus ungriai Garcia-Rodrigo, 1960]

GENUS Neorhadinorhynchus Yamaguti, 1939 [syns. Neogorgorhychus Golvan, 1960; Diplosentis fide Pichelin and Cribb 2001]

SPECIES

N. aspinosus (Fukui et Morisita, 1937) Yamaguti, 1939

(type species)

[syns. Rhadinorhynchus aspinosus Fukui et Morisita, 1937; Neogorgorhynchus aspinosus (Fukui et Morisita) Golvan, 1960; Pararhadinorhynchus aspinosus (Fukui et Morisita, 1937) Petrochenko, 1956] (nec aspinosum)

N. atlanticus Gaevskaja et Nigmatullin, 1977

N. atypicalis Amin et $\mathrm{Ha}, 2011$

N. macrospinosus Amin et Nahhas, 1994

N. madagascariensis Golvan, 1969

N. myctophumi Mordvinova, 1988

N. nudus (Harada, 1938) Yamaguti, 1939

[syns. Rhadinorhynchus nudus Harada, 1938; Neogorgorhynchus nudus (Harada, 1938) Golvan, 1960; Nipporhynchus nudus (Harada,

1938) Van Cleave et Lincicome, 1940; Echinorhynchus nudus (Harada, 1938) Petrochenko, 1956] (nec nudum)

\section{GENUS Paracavisoma Kritscher, 1957}

\section{SPECIES}

P. impudica (Diesing, 1851) Kritscher, 1957 (type species)

[syn. Echinorhynchus impudicus Diesing, 1851] (nec impudicum)

GENUS Pseudocavisoma Golvan et Houin, 1964

[syn. Rhadinorhynchoides in Yamaguti 1963]

SPECIES

P. chromitidis (Cable et Quick, 1954) Golvan et Houin, 1964 (type species)

[syns. Cavisoma chromitidis Cable et Quick, 1954; Rhadinorhynchus chromitidis (Cable et Quick, 1954) Yamaguti, 1963]

GENUS Rhadinorhynchoides Fukui et Morista, 1937

\section{SPECIES}

R. miyagawai Fukui et Morisita, 1937 (type species)

FAMILY Diplosentidae Tubangui et Masilungan, 1937

[syn. Cavisomidae in Pichelin and Cribb 2001]

SUBFAMILY Allorhadinorhynchinae Golvan, 1969

(diagnosis in Amin and Sey 1996)

GENUS Allorhadinorhynchus Yamaguti, 1959

SPECIES

A. segmentatus Yamaguti, 1959 (type species) (nec segmentatum)

GENUS Golvanorhynchus Noronha, Fabio et Pinto, 1978

SPECIES

G. golvani Noronha, Fabio et Pinto, 1978 (type species)

SUBFAMILY Diplosentinae Tubangui et Masilungan, 1937

GENUS Amapacanthus Salgado-Maldonado et Portes Santos, 2000

SPECIES

A. amazonicus Salgado-Maldonado et Portes Santos, 2000 (type species)

GENUS Diplosentis Tubangui et Masilungan, 1937

SPECIES

D. amphacanthi Tubangui et Masilungan, 1937 (type species)

D. manteri Gupta et Fatma, 1979

GENUS Pararhadinorhynchus Johnston et Edmonds, 1947

SPECIES

P. coorongensis Edmonds, 1973

P. mugilis Johnston et Edmonds, 1947 (type species)

FAMILY Echinorhynchidae Cobbold, 1876 
SUBFAMILY Circinatechinorhynchinae Bhattacharya, 2007 GENUS Circinatechinorhynchus Bhattacharya, 2007

\section{SPECIES}

C. pseudorhombi Bhattacharya, 2007 (type species)

SUBFAMILY Echinorhynchinae Cobbold, 1876

GENUS Acanthocephalus Koelreuther, 1771

[syns. Paracanthocephalus Achmerov et DombrovskajaAchmerova, 1941; Pseudoechinorhynchus Petrochenko, 1956]

SPECIES

A. acutispinus Machado Filho, 1968

A. acutulus Van Cleave, 1931

A. alabamensis Amin et Williams, 1983

[syn. Acanthocephalus etowani Williams, 1974]

(A. etowani was improperly described and named in an unpublished dissertation - Williams 1974.)

A. amini Salgado-Maldonado, 2009

A. anguillae (Müller, 1780) Lühe, 1911 (type species)

[syns. Echinorynchus anguillae Müller, 1780; E. globulosus Rudolphi, 1802; E. linstowi Hamann, 1891; E. paronai Condorelli, 1897; E. proteus Porta, 1905; Acanthocephalus paronai (Condorelli, 1897) Meyer, 1932 (fide Golvan 1960)]

A. anthuris (Dujardin, 1845) Lühe, 1911

[syn. Echinorynchus anthuris Dujardin, 1845]

A. atratus Van Cleave, 1925 (nec aratus)

[syn. Acanthocephalus lucidus (fide Harada 1935, fide Yamaguti 1939)]

A. balkanicus Bachvarov, 1974

A. clavula (Dujardin, 1845) Grabda-Kazubska et Chubb, 1968

(nec Hamann, 1892)

[syn. Echinorynchus clavula Dujardin, 1845]

A. correalimai Machado Filho, 1970

A. criniae Snow, 1971

A. curtus (Achmerov et Dombrovskaja-Achmerova, 1941)

Yamaguti, 1963

[syns. Paracanthocephalus curtus Achmerov et Dombrovskaja-Achmerova, 1941; Acanthocephalus amuriensis Kostylew, 1941]

A. dirus (Van Cleave, 1931) Van Cleave et Townsend, 1936;

[syns. Echinorynchus dirus Van Cleave, 1931; Acanthocephalus

jacksoni Bullock, 1962; A. parksidei Amin, 1975]

A. domerguei Golvan, Bygoo et Gassmann, 1972

A. echigoensis Fujita, 1920

[syns. Acanthocephalus acerbus Van Cleave, 1931; A. aculeatus Van Cleave, 1931 (fide Harada 1935); A. onchorhynchi Fujita, 1920]

A. elongatus Van Cleave, 1937

A. falcatus (Frölich, 1789) Lühe, 1911

[syn. Echinorynchus falcatus Frölich, 1789]

A. fluviatilis Paperna, 1964 (nec fluviatilus)

A. galaxii Hine, 1978 (nec 1977)

A. goaensis Jain et Gupta, 1981

A. gotoi Van Cleave, 1925

A. gracilacanthus (Meyer, 1932) Grabda et Grabda-Kazubska, 1967 [syn. Paracanthocephalus gracilacanthus (Meyer, 1932) GrabdaKazubska, 1967]

A. halongensis Amin et $\mathrm{Ha}, 2011$

A. haranti Golvan et Oliver in Golvan, 1969

A. hastae Baylis, 1944

A. japonicus (Fukui et Morisita, 1936) Petrochenko 1956

[syns. Filisoma japonicum Fukui et Morisita, 1936; Acanthocephaloides japonicus (Fukui et Morisita, 1936) Yamaguti, 1939]

A. kabulensis Datta et Soota, 1956

A. kashmirensis Datta, 1936

A. lizus Li-Minmin, 1984

A. loktakensis Shomorendra, Ranibala et Jha, 2009

A. lucii (Müller, 1776) Lühe 1911

[syns. Echinorynchus lucii Müller, 1776; E. angustus Rudolphi, 1809; E. blennii Rudolphi, 1807]
A. madagascariensis Golvan, 1965

A. manipurensis Bhattacharya, 2007

A. minor Yamaguti, 1935

A. nanus Van Cleave, 1925

A. nickoli Khan et Bilqees, 1994

A. opsariichthydis Yamaguti, 1935 (nec opsalichtydis, nec opsalichthydis) (vide Yamaguti 1939)

A. parallelotestis Achmerov et Dombrovskaja-Achmerova, 1941

A. pesteri Tadros, 1966

A. ranae (Schrank, 1788) Lühe 1911

[syns. Echinorynchus ranae Schrank, 1788; E. haeruca Rudolphi, 1809; Acanthocephalus praetextus Molin, 1858] (fide Porta 1908)

A. rauschi (Schmidt, 1969) Amin, 1985

[syn. Paracanthocephalus rauschi Schmidt, 1969; A. rauschi Golvan, 1969]

A. reunionensis Smales, Sasal et Taraschewski, 2007

A. rhinensis Amin, Thielen, Münderle, Taraschewski et Sures, 2008

A. saurius Bursey et Goldberg, 2003

A. serendibensis Crusz et Mills, 1970

A. sichuanensis Wang et Zhang, 1987

A. sinensis Van Cleave, 1937

A. srilankensis Crusz et Ching, 1976

A. tahlequahensis Oetinger et Buckner, 1976

A. tenuirostris (Achmerov et Dombrovskaja-Achmerova, 1941) Yamaguti 1963

[syn. Paracanthocephalus tenuirostris Achmerov et Dombrovskaja Achmerova, 1941]

A. tigrinae (Shipley, 1903) Yamaguti, 1963

[syn. Echinorynchus tigrinae Shipley, 1903]

A. tumescens (von Linstow, 1896) Porta, 1905

[syn. Echinorynchus tumescens von Linstow, 1896]

A. ula Lent et Santos, 1990

GENUS Anuracanthorhynchus Bursey, Vrcibradic, Hatano et Rocha, 2006

SPECIES

A. tritaxisentis Bursey, Vrcibradic, Hatano et Rocha, 2006

(type species)

GENUS Brasacanthus Thatcher, 2001

SPECIES

B. sphoeroides Thatcher, 2001 (type species)

GENUS Echinorhynchus Zoega in Müller, 1776

[syns. Metechinorhynchus Petrochenko, 1956; Pseudoechinorhynchus Petrochenko, 1956]

(The extremely variable and overlapping cement gland pattern in this originally well defined genus was the basis for splitting it to three poorly defined genera by Petrochenko 1956 or subgenera by Golvan 1960-1961. Yamaguti 1963 did not accept this arrangement; neither do we. The synonymy was established by Amin and Redlin 1980.)

SPECIES

E. abyssicola Dollfus, 1931

E. alpinus von Linstow, 1901

E. armoricanus Golvan, 1969

E. attenuatus Linton, 1888 (nec 1890, nec 1891)

E. baeri Kostylew, 1928

[syns. Echinorhynchus sevangi Dinnik, 1933; Metechinorhynchus baeri (Kostylew, 1928) Petrochenko, 1956]

E. bothniensis Zdzitowiecki et Valtonen, 1987

(Using allozyme analysis, Väinölä et al. 1994 showed that $E$. bothniensis from the northern Baltic Sea represents a complex of freshwater taxa associated with the 'glacial relic' Mysis spp. intermediate hosts.)

E. brayi Wayland, Sommerville et Gibson, 1999

E. briconi Machado Filho, 1959

[syn. Metechinorhynchus brinconi (Machado Filho, 1959) Golvan, 1969]

E. calloti Golvan, 1969 
E. canyonensis Huffman et Kliever, 1977 (nec Kleiver)

E. cestodicola von Linstow, 1905

E. cherchiae Monticelli, 1889 (nec chierchiae)

E. cinctulus (Porta, 1905) comb. n.

[syns. Pseudoechinorhynchus cinctulus (Porta, 1905) Petrochenko, 1956; E. borealis von Linstow, 1901]

E. coregoni Linkins in Van Cleave, 1919

[syn. Echinorhynchus (Metechinorhynchus) coregoni (Linkins in Van Cleave, 1919) Golvan, 1994]

E. cotti Yamaguti, 1939

E. cryophilus (Sokolovskaja, 1962) Amin, 1985

[syn. Metechinorhynchus cryophilus Sokolovskaja, 1962]

E. dissimilis Yamaguti, 1939

E. gadi Zoega in Müller, 1776 (type species)

[syns. Echinorhynchus acus Rudolphi, 1802; E. gadicallariae Viborg, 1795; E. gadiverentis Rathke, 1799; E. hepaticola von Linstow, 1901; E. lineolatus Müller, 1777; E. lophii Gmelin, 1791; E. socialis Leidy, 1851; E. vancleavei Golvan, 1969; E. wachniae Rudolphi, 1819] (Väinölä et al. 1994 demonstrated strong allozyme divergence between the marine E. gadi and the fresh- brakish-water E. salmonis supporting the genetic distinction between these two taxa. Wayland et al. 2005 used electrophoresis to detect the existence of two reproductively isolated species, A and B, within the E. gadi complex. The two species can be discriminated in graphical and cluster analysis of hook morphometrics. Reproductive isolation was not a function of differential host specifity or seasonal differences in mating time. Sobecka et al. 2012 compared populations of $E$. gadi from the Atlantic cod, Gadus morhua Linneaus in the Baltic Sea and the North Atlantic morphometrically and genetically using polymerase chain reactionrestriction fragment length polymorphism and selected PCR products. The molecular analysis showed the nucleotide sequesnces of $E$. gadi rDNA from cod collected from all sites to be identical. Morphometric analysis, however, demonstrated the separation of E. gadi into two groups corresponding to the separation of cod into two subspecies, G. m. morhue in the Atlantic and G. m. callarias in the Baltic.)

E. gomesi Machado Filho, 1948

[syn. Metechinorhynchus gomesi (Machado Filho, 1948) Petrochenko, 1956]

E. gracilis Machado Filho, 1948

E. gymnocyprii Liu, Wang et Yang, 1981

E. hexagrammi Beava, 1965

E. indicus Chandra, Hanumantha-Rao et Shyamasundari, 1982

E. jucundus Travassos, 1923

[syn. Metechinorhynchus jucundum (Travassos, 1923) Petrochenko, 1956]

E. kushiroensis Fujita, 1921

[syn. Metechinorhynchus kushiroensis (Fujita, 1921) Petrochenko, 1956]

E. lageniformis Ekbaum, 1938

[syn. Metechinorhynchus lageniformis (Ekbaum,1938) Petrochenko, 1956]

E. laurentianus Ronald, 1957

E. leidyi Van Cleave, 1924

[syn. Metechinorhynchus leidyi (Van Cleave, 1924) Golvan, 1969]

E. lenoki Achmerov et Dombrovskaja-Achmerova, 1941

[syn. Pseudoechinorhynchus lenoki (Achmerov et Dombrovskaja-

Achmerova, 1941) Petrochenko, 1956]

E. lesteri Smales, 2012

E. longiproboscis Rodjuk, 1986

E. lotellae Yamaguti, 1939

E. malacocephali (Parukhin, 1985) comb. n.

[syn. Metechinorhynchus malacocephali Parukhin, 1985]

E. melanoglaeae Dollfus, 1960

E. monticelli Porta, 1904

[syn. Pseudoechinorhynchus monticelli (Porta, 1904) Petrochenko, 1956]

E. muraenolepisi (Rodjuk, 1984) comb. n.

[syn. Metechinorhynchus muraenolepisi Rodjuk, 1984]

E. oblitus Golvan, 1969

E. orientalis Kaw, 1951

E. paranensis Machado Filho, 1959

[syn. Metechinorhynchus paranensis (Machado Filho, 1959) Golvan, 1969]
E. parasiluri Fukui, 1929

[syn. Pseudoechinorhynchus parasiluri (Fukui, 1929) Petrochenko, $1956]$

E. petrotschenkoi (Rodjuk, 1984) comb. n.

[syns. Metechinorhynchus petrotschenkoi Rodjuk, 1984; Echinorhynchus georgianus (Rodjuk, 1986) Zdzitowiecki, 1989; E. nototheniae (Zdzitowiecki, 1986) Zdzitowiecki ,1989]

E. rhenanus (Golvan, 1969) Amin, 1985

[syn. Metechinorhynchus rhenanus Golvan, 1969]

E. salmonis Müller, 1784

[syn. E. alpinus von Linstow, 1901; E. coregoni Linkins in Van Cleave, 1919; E. murenae Bosc, 1802; E. pachysomus Creplin, 1839; E. phoenix Schneider, 1903); Metechinorhynchus alpinus (von Linstow, 1901) Petrochenko, 1956; M. salmonis (Müller, 1784) Petrochenko, 1956] (This species is apparently not found in British and Irish freshwater fishes. Chubb 2004 examined collections of 'E. salmonis' from Britan and Ireland deposited in the Natural History Museum, London, and reidentified them as Acanthocephalus clavula and Acanthocephalus lucii, respectively. The intermediate host, Pontoporeia affinis, in northern Europe is absent from the British Isles.)

E. salobrensis Machado Filho, 1948

[syn. Metechinorhynchus salobrensis (Machado Filho, 1948) Golvan, 1969]

E. sebastolobi Kovalenko, 1986

E. sevani Dinnik, 1932

[syn. Echinorhynchus (metechinorhynchus) sevani (Dinnik, 1932) Golvan, 1994] (nec sevangi)

E. theragrae Dydenko, 1992

E. trachyrinci Wayland, Gibson et Sommerville, 1997

E. truttae Schrank, 1788

[syn. Metechinorhynchus truttae (Schrank, 1788) Petrochenko, 1956]

E. vancleavei Golvan, 1969

[syn. Echinorhynchus (Echinorhynchus) vancleavei Golvan, 1969]

E. veli George et Nadakal, 1981 (nec 1978)

E. yamagutii Golvan, 1969

GENUS Frilloechinorhynchus (Gupta et Naqvi, 1986) Bhattacharya, 2007

SPECIES

F. meyeri (Gupta et Naqvi, 1986) Bhattacharya, 2007

[syn. Echinorhynchoides meyeri Gupta et Naqvi, 1986]

GENUS Pilum Williams, 1976

SPECIES

P. pilum Williams, 1976 (type species)

GENUS Pseudoacanthocephalus Petrochenko, 1956

(Amin et al. 2008 discussed and continued to justify the validity of Pseudoacanthocephalus, and provided a key to valid species. Tkach et al. 2013 used comparative analysis of nuclear ribosomal rRNA sequences encompassing the 3 ' end of $18 \mathrm{~S}$ nuclear rDNA gene, internal transcribed spacer region (ITS1+5.8S+ITS2), and 5' end of the $28 \mathrm{~S}$ gene to demonstrate significant differences between $P$. nickoli and $P$. smalesi as well as between these two species and closely related species from China and Vietnam.)

SPECIES

P. betsileo Golvan, Houin et Bygoo, 1969

P. bigueti (Houin, Golvan et Bygoo, 1965) Golvan, 1969

[syn. Acanthocephalus bigueti Houin, Golvan et Bygoo, 1965]

P. bufonicola (Kostylew, 1941) Petrochenko, 1956

[syn. Acanthocephalus bufonicola Kostylew, 1941]

(nec bufonincola)

P. bufonis (Shipley, 1903) Petrochenko, 1956 (type species)

[syns. Echinorhynchus bufonis Shipley, 1903; Acanthocephalus bufonis (Shipley, 1903) Southwell et MacFie, 1925 sensu Petrochenko, 1953; A. breviprostatus Kennedy, 1982; A. sinensis Van Cleave, 1937] P. caspanensis (Fernández et Ibarra Vidal, 1992) Arredondo et Gil de Pertierra, 2009

[syn. Acanthocephalus caspanensis Fernández et Ibarra Vidal, 1992]

P. caucasicus (Petrochenko, 1953) Petrochenko, 1956

[syn. Acanthocephalus caucasicus Petrochenko, 1953]

P. elongatus (Van Cleave, 1937) Petrochenko, 1958

P. lutzi (Hamann, 1891) Arredondo et Gil de Pertierra, 2009 
[syns. Echinorhynchus lutzi Hamann, 1891; Acanthocephalus lutzi (Hamann, 1891) Meyer, 1932; Acanthocephalus saopaulensis Smales, 2007; Pseudoacanthocephalus saopaulensis (Smales, 2007) Arredondo et Gil de Pertierra, 2009]

P. nguyenthileae Amin, Ha et Heckmann, 2008

P. nickoli Tkach, Lisitsyna, Crossley, Binh et Bush, 2013

P. paratiensis Bhattacharya, 2000

P. perthensis Edmonds, 1971

P. rauschi Gupta et Fatma, 1986

P. reesei Bush, Duzynski et Nickol, 2009

P. rhampholeonotos Smales, 2005

P. shillongensis Bhattacharya, 1999

P. smalesi Tkach, Lisitsyna, Crossley, Binh et Bush, 2013

P. xenopeltidis (Shipley, 1903) Golvan, 1969

[syn. Echinorhynchus xenopeltidis Shipley, 1903]

FAMILY Fessisentidae Van Cleave, 1931

GENUS Fessisentis Van Cleave, 1931

SPECIES

F. acutulus (Van Cleave, 1931) McAlpine, 1997

[syn. Acanthocephalus acutulus Van Cleave, 1931]

F. fessus Van Cleave, 1931 (type species)

F. friedi Nickol, 1972

[syn. Fessisentis vancleavei sensu Haley et Bullock, 1953]

F. necturorum Nickol, 1967

F. tichiganensis Amin, 1980

F. vancleavei (Hughes et Moore, 1943) Nickol, 1972 (nec vancleavi) [syn. Acanthocephalus vancleavei Hughes et Moore, 1943]

FAMILY Heteracanthocephalidae Petrochenko, 1956

SUBFAMILY Aspersentinae Golvan, 1960

GENUS Aspersentis Van Cleave, 1929

[syn. Heteracanthocephalus Petrochenko, 1956]

SPECIES

A. austrinus Van Cleave, 1929

[syns. Heteracanthocephalus hureaui (Dollfus, 1964) Zdzitowiecki, 1986; Aspersentis megarhynchus von Linstow, 1892) Golvan, 1960; A. wheeleri (Baylis, 1929) Chandler, 1934; Echinorhynchus megarhynchus von Linstow, 1892; Rhadinorhynchus wheeleri Baylis, 1929]

A. dissosthychi (Parukhin, 1989) comb. n.

[syn. Heteracanthocephalus dissosthychi Parukhin, 1989]

A. johni (Baylis, 1929) Chandler, 1934

[syn. Rhadinorhynchus johni Baylis, 1929]

A. megarhynchus (von Linstow, 1892) Golvan, 1960 (type species) [syn. Aspersentis wheeleri (Baylis, 1929) Chandler, 1934]

A. minor Edmonds et Smales, 1991

A. peltorhampi (Baylis, 1944) Pichelin, Smales et Bray, 2002

[syns. Rhadinorhynchus peltorhampi Baylis, 1944; Heteracanthocephalus peltorhampi (Baylis, 1944) Petrochenko, 1956]

A. zanclorhynchi (Johnston et Best, 1937) Smales, 1996

[syn. Echinorhynchus zanclorhynchi Johnston et Best, 1937] (nec zanchlorhynchi, nec zanchlorhynchus)

SUBFAMILY Heteracanthocephalinae Petrochenko, 1956

GENUS Bullockrhynchus Chandra, Hanumantha Rao et Shyamasundari, 1985

SPECIES

B. indicus Chandra, Hanumantha Rao et Shyamasundari, 1985 (type species)

GENUS Sachalinorhynchus Krotov et Petrochenko in Petrochenko, 1956

SPECIES

S. skrjabini Krotov et Petrochenko, 1956 in Petrochenko, 1956 (type species)

FAMILY Illiosentidae Golvan, 1960
GENUS Brentisentis Leotta, Schmidt et Kuntz, 1982

\section{SPECIES}

B. chongqingensis Wei, 1998

B. uncinus Leotta, Schmidt et Kuntz, 1982 (type species)

B. yangtzensis $\mathrm{Yu}$ et $\mathrm{Wu}, 1989$

GENUS Dentitruncus Sinzar, 1955

SPECIES

D. truttae Sinzar, 1955 (type species)

GENUS Dollfusentis Golvan, 1969

SPECIES

D. bravoae Salgado-Maldonado, 1976

D. chandleri Golvan, 1969

[syns. Telosentis tenuicornis (Linton, 1905) Van Cleave, 1947, in part; Echinorhynchus pristis tenuicornis sensu Linton, 1905; Rhadinorhynchus tenuicornis (Linton, 1905) Van Cleave, 1918; R. tenuicornis sensu Chandler, 1934]

D. ctenorhynchus (Cable et Linderoth, 1963) Golvan, 1969 [syn. Illiosentis ctenorhynchus Cable et Linderoth, 1963] D. heteracanthus (Cable et Linderoth, 1963) Golvan, 1969 [syn. Illiosentis heteracanthus (Cable et Linderoth, 1963) Monks et Pulido-Flores, 2002]

D. longispinus (Cable et Linderoth, 1963) Golvan, 1969 (type species)

[syns. Telosentis tenuicornis (Linton, 1892) Van Cleave, 1947; Rhadinorhynchus tenuicornis (Linton, 1891) Van Cleave, 1947; Illiosentis longispinus Cable et Linderoth, 1963]

D. salgadoi Monks, Aleman-Garcia et Pulido-Flores, 2008

GENUS Goacanthus Gupta et Jain, 1980

SPECIES

G. panajiensis Gupta et Jain, 1980 (type species)

GENUS Indorhynchus Golvan, 1969

SPECIES

I. indicus (Tripathi, 1959) Golvan, 1969 (type species)

[syn. Rhadinorhynchus indicus Tripathi, 1959]

I. pseudobagri Wang, 1988

GENUS Koronacantha Monks et

Pérez-Ponce de León, 1996

SPECIES

K. mexicana Monks et Pérez-Ponce de León, 1996 (type species)

K. pectinarius (Van Cleave, 1940) Monks et Pérez-Ponce de León, 1996

[syn. Tegorhynchus pectinarius Van Cleave, 1940]

GENUS Metarhadinorhynchus Yamaguti, 1959

SPECIES

M. cyprini (Yin, 1961) Wang, 1986

[syns. Rhadinorhynchus arri Wang, 1966; R. cyprini (Wang, 1966) Wang, 1986]

M. echeneisi Gupta et Sinha, 1991

M. lateolabracis Yamaguti, 1959 (type species)

M. thapari Gupta et Gupta, 1975

M. valiyathurae Nadakal, John et Jacob, 1990

GENUS Paradentitruncus Moravec et Sey, 1989

SPECIES

P. longireceptaculis Moravec et Sey, 1989 (type species)

GENUS Pseudorhadinorhynchus Achmerov et Dom-

brovskaja-Achmerova, 1941

[syn. Hemirhadinorhynchus Krotov et Petrochenko, 1956]

SPECIES

P. cinereus Gupta et Naqvi, 1983

P. cochinensis Gupta et Naqvi, 1983 
P. deeghai Saxena, 2003

P. dhari Kumar, 1992

P. dussamicitatum Gupta et Gupta, 1971

P. ernakulensis Gupta et Gupta, 1971

P. guptai Gupta et Sinha, 1993

P. leuciscus (Krotov et Petrochenko, 1956) Golvan, 1969

[syn. Hemirhadinorhynchus leuciscus Krotov et Petrochenko, 1956]

P. machidai Kumar, 1992

P. markewitschi Achmerov et Dombrovskaja-Achmerova, 1941 (type species) (nec markewitchi)

P. mujibi Gupta et Naqvi, 1983

P. nandai Gupta et Sinha, 1993

P. orissai Gupta et Fatma, 1985

P. pseudaspii Achmerov et Dombrovskaja-Achmerova, 1941

P. salmothymi Rukavina et Goric in Cankovic, Delic, Kiškarolj et Rukavina, 1968

P. samegaiensis Nakajima, 1975 (nec samegainensis)

P. srivastavai Gupta et Fatma, 1985

P. vietnamensis Moravec et Sey, 1989

GENUS Tegorhynchus Van Cleave, 1921

[syn. Illiosentis Van Cleave et Lincicome, 1939]

\section{SPECIES}

T. africanus (Golvan, 1955) Amin, 1985

[syn. Illiosentis furcatus africanus Golvan, 1955]

T. brevis Van Cleave, 1921 (type species)

T. cetratus (Van Cleave, 1945) Bullock et Mateo, 1970

[syn. Illiosentis cetratus Van Cleave, 1945] (nec centratus)

T. edmondsi (Golvan, 1960) Amin, 1985

[syn. Illiosentis edmondsi Golvan, 1960]

T. furcatus (Van Cleave et Lincicome, 1939) Bullock et Mateo, 1970

[syn. Illiosentis furcatus Van Cleave et Lincicome, 1939]

T. holospinosus Amin et Sey, 1996

T. pectinarius Van Cleave, 1940

[syn. Telosentis pectinarius Van Cleave, 1940]

GENUS Telosentis Van Cleave, 1923

\section{SPECIES}

T. australiensis Edmonds, 1964

T. exiguus (von Linstow, 1901) Van Cleave, 1923

[syn. Echinorhynchus exiguus von Linstow, 1901]

T. Iutianusi Gupta et Gupta, 1990

T. mizellei Gupta et Fatma, 1988

T. molini Van Cleave, 1923 (type species)

[syns. Echinorhynchus acanthosoma Westrumb, 1821; E. atherinae Rudolphi, 1819]

FAMILY Isthmosacanthidae Smales, 2012

\section{GENUS Isthmosacanthus Smales, 2012}

\section{SPECIES}

Isthmosacanthus fitzroyensis Smales, 2012 (type species)

(The proposed relegation of the rhadinorhynchid genera Gorgorhynchoides Cable et Linderoth, 1963 and Golvanorhynchus Noronha, Fabio et Pinto, 1978 to Isthmosacanthidae by Smales 2012 was based on having in common six cement glands and similar shape of the proboscis, the extention of proboscis receptacle, anterior trunk spines, elongate lemnisci, and trunk bulb. Many of these features are also shared by other rhadinorhynchid genera. The reference by Smales 2012 that Rhadinorhynchidae is restricted to genera with only four cement glands is not accurate. Rhadinorhynchidae has 2-8 cement glands, variable proboscis shapes, variable lemniscal and receptacle lengths, and variable trunk spination and swellings - Yamaguti 1963, Amin et al. 2011b. Species of Rhadinohynchus that have other than four cement glands include $R$. dollfusi and $R$. echeneisi (with two glands) and $R$. capensis and $R$. trivandricus (with seven or eight glands - Amin et al. 2011b. The Smales 2012 proposal is thus not accommodated.)

FAMILY Pomphorhynchidae Yamaguti, 1939

[syn. Spirorhynchidae Harada, 1935]
GENUS Longicollum Yamaguti, 1935

[syns. Spiracanthorhynchus Harada, 1938; Spirorhynchoides Strand, 1942; Spirorhynchus Harada, 1935]

SPECIES

L. alemniscus (Harada, 1935) Fukui et Morisita, 1938

[syns. Spirorhynchus alemniscus Harada, 1935; Longicollum minor Fukui et Morisita, 1936; Spiracanthorhynchus alemniscus (Harada, 1935) Harada, 1938]

L. cadenati Gupta et Naqvi, 1984

L. chabanaudi Dollfus et Golvan, 1963

L. dattai Saxena, Johri et Gupta, 2008

L. edmondsi Golvan, 1969

[syn. Longicollum pagrosomi sensu Johnston et Edmonds, 1951]

L. engraulisi Gupta et Fatma, 1985

L. indicum Gupta et Gupta, 1970

L. lutiani Jain et Gupta, 1980 (nec lutjani)

L. noellae Golvan, 1969

L. pagrosomi Yamaguti, 1935 (type species) (nec pagrosoma)

L. psettodai Gupta et Gupta, 1980 (nec psettodsai)

L. quiloni Gupta et Naqvi, 1984

L. riouxi Golvan, 1969

GENUS Paralongicollum Amin, Bauer et Sidorov, 1991

\section{SPECIES}

P. nemacheili Amin, Bauer et Sidorov, 1991 (type species)

P. sergenti (Choquette et Gayot, 1952) Amin, 1991

[syns. Tenuiproboscis sergenti Choquette et Gayot, 1952; Longicollum sergenti (Choquette et Gayot, (1952) Golvan, 1969]

GENUS Pomphorhynchus Monticelli, 1905

\section{SPECIES}

P. bosniacus Kistaroly et Cankovic, 1969

P. bufonis Fotedar, Duda et Raina, 1970

P. bulbocolli Linkins in Van Cleave, 1919

P. bullocki Gupta et Lata, 1968

P. cylindrica Wang et Gue, 1983 (nec cylinderica)

P. dubious Kaw, 1941

P. francoisae Golvan, 1969

P. intermedius Engelbrecht, 1957

P. jammuensis Fotedar et Dhar, 1977

P. kashmirensis Kaw, 1941

P. kawi Fotedar, Duda et Raina, 1970

P. kostylewi Petrochenko, 1956

P. laevis (Zoega in Müller, 1776) Van Cleave, 1924 (type species) [syn. Echinorhynchus proteus Westrumb, 1821] (nec leave)

(Based on isoenzyme analysis, Dudiňák and Snábel 2001 described genetic differences between the $P$. laevis populations of the Slovak and Czech Republics. Geographical isolation has apparently produced distinct genetic forms irrespective of host species. PerrotMinnot 2004 demonstrated a high level of sequence divergence at ITS1, ITS2 and cytochrome c oxidase between smooth and wrinkled cystacanths of $P$. laevis, which corresponded with phototactile behavioral differences in gammarid hosts. She speculated that the smooth type corresponds to $P$. laevis and the wrinkled type to $P$. tereticollis, a former synonym of $P$. laevis. O'Mahony et al. 2004 distinguished populations of $P$. laevis from western Ireland and southern England using the position of the stoutest proboscis hook and the ratio of numbers of anterior to posterior hooks. Špakulová et al. 2011 distinguished between $P$. laevis and $P$. tereticollis based on differences in proboscis armature and gene sequencing using ITS1, ITS2 and COI.)

P. lucyi Williams et Rogers, 1984

P. megacanthus Fotedar et Dhar, 1977

P. moyanoi Olmos et Habit, 2007

P. omarsegundoi Arredondo et Gil de Pertierra, 2010

P. oreini Fotedar et Dhar, 1977 (nec 1974 fide Golvan 1994)

P. orientalis Fotedar et Dhar, 1977 (nec 1974 fide Golvan 1994)

P. patagonicus Ortubay, Ubeda, Semenas et Kennedy, 1991

P. perforator (von Linstow, 1908) Meyer, 1932 
[syn. Echinorhynchus perforator von Linstow, 1908]

P. rocci Cordonnier et Ward, 1967

P. sebastichthydis Yamaguti, 1939

P. sphaericus Pertierra, Spatz et Doma, 1996

[syn. Pomphorhynchus patii Lunaschi, 1997]

P. spindletruncatus Amin, Abdullah et Mhaisen, 2003

P. tereticollis (Rudolphi, 1809) Meyer, 1932

[syns. Echinorhynchus tereticollis Rudolphi, 1809; E. attenuatus Müller, 1779; Pomphorhynchus dobulae Schrank, 1790; E. longicollis Pallas, 1782, in part; E. piscinus Zeder, 1900, in part] (nec tereticolle)

P. tori Fotedar et Dhar, 1977 (nec 1974 fide Golvan 1994)

P. yamagutii Schmidt et Higgins, 1973

P. yunnanensis Wang, 1981

GENUS Pyriproboscis Amin, Abdullah et Mhaisen, 2003

SPECIES

P. heronensis (Pichelin, 1997) Amin, Abdullah et Mhaisen, 2003

(type species)

[syn. Pomphorhynchus heronensis Pichelin, 1997]

GENUS Tenuiproboscis Yamaguti, 1935

SPECIES

T. bilqeesae Gupta et Naqvi, 1992

T. clupei Gupta et Gunjan-Sinh, 1992

T. edmondi Gupta et Naqvi, 1992

T. ernakulensis Gupta et Naqvi, 1992

T. guptai Gupta et Sinha, 1989

T. meyeri Saxena et Gupta, 2007

T. misgurni Yamaguti, 1935 (type species)

FAMILY Rhadinorhynchidae Lühe, 1912

[syns. Gorgorhynchidae Van Cleave et Lincicome, 1940; Micracanthorhynchinidae Yamaguti, 1963; Raorhynchidae Tripathi, 1959]

SUBFAMILY Golvanacanthinae Paggi et Orecchia, 1972

GENUS Golvanacanthus Paggi et Orecchia, 1972

\section{SPECIES}

G. blennii Paggi et Orecchia, 1972 (type species)

[syn. Golvanacanthus problematicus Mordinova et Parukhin, 1978] (nec Mordvinova)

SUBFAMILY Gorgorhynchinae Van Cleave et Lincicome, 1940

[syn. Leptorhynchoididae Witenberg, 1932]

GENUS Australorhynchus Lebedev, 1967

SPECIES

A. tetramorphacanthus Lebedev, 1967 (type species)

GENUS Cleaveius Subrahmanian, 1927

[syn. Mehrarhynchus Datta, 1940]

SPECIES

C. circumspinifer Subrahmanian, 1927 (type species)

(nec circulspinifer)

C. clupei (Gupta et Sinha, 1992) comb. n.

[syn. Mehrarhynchus clupei Gupta et Sinha, 1992]

C. durdanae Kumar, 1992

C. fotedari (Gupta et Naqvi, 1980) comb. $\mathbf{n}$.

[syn. Mehrarhynchus fotedari Gupta et Naqvi, 1980]

C. inglisi (Gupta et Fatma, 1987) Golvan, 1994

[syn. Mehrarhynchus inglisi Gupta et Fatma, 1987]

C. leiognathi Jain et Gupta, 1979

C. longirostris Moravec et Sey, 1989

C. mysti (Sahay et Sinha, 1971) Amin, 1985

[syn. Mehrarhynchus mysti Sahay et Sinha, 1971]

C. portblairensis Jain et Gupta, 1979
C. prashadi (Datta, 1940) Golvan, 1969

[syn. Mehrarhynchus prashadi Datta, 1940]

C. puriensis (Gupta et Sinha, 1992) comb. n.

[syn. Mehrarhynchus puriensis Gupta et Sinha, 1992]

C. secundus (Tripathi, 1959) Golvan, 1969

[syn. Mehrarhynchus secundus Tripathi, 1959]

C. singhai (Gupta et Fatma, 1987) Golvan, 1994

[syn. Mehrarhynchus singhai Gupta et Fatma, 1987]

C. thapari (Gupta et Naqvi, 1980) comb. n.

[syn. Mehrarhynchus thapari Gupta et Naqvi, 1980]

GENUS Edmondsacanthus Smales, 2009

SPECIES

E. blairi Smales, 2009 (type species)

GENUS Gorgorhynchoides Cable et Linderoth, 1963

(The placement of Gorgorhynchoides within the Echinorhynchida and not Polymorphida, based on analyses of nuclear 18S rDNA sequences, was questioned by Verweyen et al. 2011.)

\section{SPECIES}

G. bullocki Cable et Mafarachisi, 1970

G. cablei (Gupta et Fatma, 1987) Bhattacharya, 2007

[syn. Neogorgorhynchoides cablei Gupta et Fatma, 1987]

G. elongatus Cable et Linderoth, 1963 (type species)

G. epinepheli Wang, 1986

G. golvani (Chandra, Hanumantha et Shyamasundari, 1984) Bhattacharya, 2007

[syn. Paracanthocephaloides golvani Chandra, Hanumantha et Shyamasundari, 1984]

G. indicus Bhattacharya et Banerjee, 2003

G. lintoni Cable et Mafarachisi, 1970

G. orientalis Wang, 1986

[syn. Sphaerirostris orientalis fide Wang 1986]

GENUS Gorgorhynchus Chandler, 1934

[syn. Neoacanthorhynchus Morisita, 1937]

G. celebensis (Yamaguti, 1954) Golvan, 1969

[syn. Rhadinorhynchus celebensis Yamaguti, 1954]

G. clavatus Van Cleave, 1940

[syn. Gorgorhynchus cablei Golvan, 1969]

G. lepidus Van Cleave, 1940

G. medius (Linton, 1908) Chandler, 1934 (type species)

[syns. Echinorhynchus medius Linton, 1907; Rhadinorhynchus medius (Linton, 1908) Van Cleave, 1918; Gorgorhynchus gibber Chandler, 1934]

G. nemipteri Parukhin, 1973

G. ophiocephali Furtado et Lau, 1971

G. polymixiae Kovalenko, 1981

G. robertdollfusi Golvan, 1956

G. satoi (Morisita, 1937) Yamaguti, 1963

[syns. Neoacanthorhynchus satoi Morisita, 1937; Gorgorhynchoides satoi (Morisita, 1937) Wang, 1966]

G. tonkinensis Amin et $\mathrm{Ha}, 2011$

G. trachinotus Noronha, Vicente, Pinto et Fabio, 1986

G. valiyanthurae (Anthony et al., 1990) Bhattacharya, 2007 [syn.

Metarhadinorhynchus valiyanthurae Anthony et al., 1990; another species with the same name was described by Nadakal, John et Jacob also in 1990]

GENUS Leptorhynchoides Kostylew, 1924

[syn. Pleurorhynchus Nau, 1787]

(The monophyly of Rhadinorhynchidae was challenged by molecular and morphological phylogenies. García-Varela and González-Oliver 2008 placed Leptorhynchoides and Pseudoleptorhynchoides in Illiosentidae based on nuclear ribosomal DNA and mitochondrial cytochrome c oxidase gene.)

SPECIES

L. aphredoderi Buckner et Buckner, 1976

L. polycristatus Amin, Heckmann, Halajian et El-Naggar, 2013

L. plagicephalus (Westrumb, 1821) Kostylew, 1924 (type species) 
[syns. Echinorhynchus plagicephalus Westrumb, 1821; E. husonis Rudolphi, 1819; E. acipenseris rutheni Rudolphi, 1819]

L. thecatus (Linton, 1891) Kostylew, 1924

[syn. Echinorhynchus thecatus Linton, 1891]

(Steinauer and Nickol 2007 detected cryptic speciation within populations of Leptorhynchoides thecatus based on sequences of the cox 1 gene and the internal transcribed spacer region, host use patterns and alternate transmission pathways.)

GENUS Metacanthocephaloides Yamaguti, 1959

\section{SPECIES}

M. zebrini Yamaguti, 1959 (type species)

GENUS Metacanthocephalus Yamaguti, 1959

\section{SPECIES}

M. campbelli (Leiper et Atkinson, 1914) Golvan, 1969

[syns. Leptorhynchoides campbelli (Leiper et Atkinson, 1914) Johnston et Best, 1937 in part; Echinorhynchus campbelli Leiper et Atkinson, 1914; E. rennicki Leiper et Atkinson, 1914; Metechinorhynchus campbelli (Leiper et Atkinson, 1914) Petrochenko, 1956]

M. dalmori Zdzitowiecki, 1983

M. johnstoni Zdzitowiecki, 1983

[syn. Leptorhynchoides campbelli (Leiper et Atkinson, 1914 sensu Johnston et Best, 1937, in part]

M. ovicephalus (Zhukov, 1963) Golvan, 1969

[syn. Leptorhynchoides ovicephalus (Zhukov, 1963) Golvan, 1969]

M. pleuronichthydis Yamaguti, 1959 (type species)

M. rennicki (Leiper et Atkinson, 1914)

[syns. Echinorhynchus rennicki Leiper et Atkinson, 1914; E. debenhami Leiper et Atkinson, 1914; Leptorhynchoides debenhami Leiper et Atkinson, 1914 sensu Johnston et Best, 1937]

GENUS Micracanthorhynchina Strand, 1936

[syns. Bolbosentis Belous, 1952; Micracanthocephalus Harada, 1938; Micracanthorhynchus Harada, 1935]

SPECIES

M. chandrai Bhattacharya, 2007

[syn. Hanumantharaorhynchus hemirhamphi Chandra, 1983]

M. cynoglossi Wang, 1980

M. dakusuiensis (Harada, 1938) Ward, 1951

M. golvani Gupta et Gunjan-Sinha, 1992

M. hemiculturus Demshin, 1965 (nec hemicultrus)

M. hemirhamphi (Baylis, 1944) Ward 1951

[syn. Micracanthocephalus hemirhamphi Baylis, 1944]

M. indica Farooqi, 1980

M. kuwaitensis Amin et Sey, 1996

M. lateolabracis Wang, 1980

M. motomurai (Harada, 1935) Ward, 1951 (type species)

[syn. Micracanthorhynchus motomurai Harada, 1935]

M. sajori (Belous, 1952) Golvan, 1969

[syn. Bolbosentis sajori Belous, 1952]

GENUS Paracanthorhynchus Edmonds, 1967

\section{SPECIES}

P. galaxiasus Edmonds, 1967 (type species)

GENUS Pseudauchen Yamaguti, 1963

\section{SPECIES}

P. epinepheli (Yamaguti, 1939) Yamaguti, 1963 (type species) [syns. Rhadinorhynchus epinepheli Yamaguti, 1939; Gorgorhynchus epinephali (Yamaguti, 1939) Golvan, 1960] (nec epinephali)

GENUS Pseudoleptorhynchoides Salgado-Maldonado, 1976 SPECIES

\section{P. lamothei Salgado-Maldonado, 1976 (type species)}

GENUS Sclerocollum Schmidt et Paperna, 1978

(We regard as untenable the proposal by Pichelin and Cribb 2001 to synonymies Sclerocollum and Neorhadinorhynchus with Diplosentis under Cavisomidae based on variable, inconsistent or questionable cement gland patterns and/or texture of the tegument. This proposal necessitated another synonymy of Diplosentidae with Cavisomidae causing confused disposition of the other genera of Diplosentidae and other uncertain or questionable relegations. Other authors, e.g. Hassanine 2006, recognized the validity of Sclerocollum.)

SPECIES

S. robustum (Edmonds, 1964) Schmidt et Paperna 1978

[syns. Neogorgorhynchus robustus Edmonds, 1964; Neorhadinorhynchus robustus (Edmonds, 1964) Johnston et Edmonds, 1964]

S. rubrimaris Schmidt et Paperna, 1978 (type species)

S. saudi Al-Jahdali, 2010

SUBFAMILY Rhadinorhynchinae Lühe, 1912

GENUS Cathayacanthus Golvan, 1969

SPECIES

C. bagarii Moravec et Sey, 1989

C. exilis (Van Cleave, 1928) Golvan, 1969 (type species)

[syn. Rhadinorhynchus exilis Van Cleave, 1928]

GENUS Corynosomoides Wang et Zhang, 1987

SPECIES

C. hemibargi Wang et Zhang, 1987 (type species)

GENUS Megistacantha Golvan, 1960

SPECIES

M. horridum (Lühe, 1912) Golvan, 1960 (type species)

[syn. Rhadinorhynchus horridus Lühe, 1912]

GENUS Neogorgorhynchoides Gupta et Fatma, 1987

SPECIES

N. cablei Gupta et Fatma, 1987 (type species)

GENUS Paragorgorhynchus Golvan, 1957

SPECIES

P. albertianus Golvan, 1957 (type species)

P. chariensis Troncy, 1970

GENUS Pseudogorgorhynchus Moravec, Wolter et Körting, 2000

SPECIES

P. arii Moravec, Wolter et Körting, 2000 (type species)

GENUS Raorhynchus Tripathi, 1959

(Raorhynchus and Rhadinorhynchus are primarily separated based on differences in trunk spination and the position of the female gonopore. Amin et al. 2011b described two new species of Rhadinorhynchus and their revision of that genus exposed extreme degrees of variability in these two traits sufficient to show that the lines of separation between these two genera are now sufficiently blurred to reconsider the present distinct status of each of these two genera. Based on these differences alone, Raorhynchus should be considered as a junior synonym to Rhadinorhynchus pending a revision of the species of Raorhynchus. That revision remains wanting.)

SPECIES

R. cadenati Gupta et Gunjan-Sinh, 1992

R. guptai Gupta et Kumar, 1987

$\boldsymbol{R}$. inexspectatus Golvan, 1969 (nec inexpectatus)

R. megalaspisi Wang, Wang et Wu, 1993

R. meyeri (Heinze, 1934) Golvan, 1969

[syn. Rhadinorhynchus meyeri Heinze, 1934]

R. polynemi Tripathi, 1959

R. schmidti George et Nadakal, 1978

R. terebra (Rudolphi, 1819) Tripathi, 1959 (type species) [syns. Echinorhynchus terebra Rudolphi, 1819; Rhadinorhynchus terebra (Rudolphi, 1819) Lühe, 1911]

R. thapari Gupta et Fatma, 1981

GENUS Rhadinorhynchus Lühe, 1911

[syns. Echinosoma Porta, 1907; Nipporhynchus Chandler, 1934; Protorhadinorhynchus Petrochenko, 1956] 
(Amin et al. 2011b revised Rhadinorhynchus, provided a list of 30 invalid species and a key to the 38 valid species based initially on the distribution of trunk spines.)

SPECIES

R. africanus (Golvan, Houin et Deltour, 1963) Golvan, 1969 [syn. Nipporhynchus africanus Golvan, Houin et Deltour, 1963]

R. atheri (Farooqui, 1981) Amin, 1985

[syn. Nipporhynchus atheri Farooqui, 1981]

R. bicircumspinis Hooper, 1983

R. cadenati (Golvan et Houin, 1964) Golvan, 1969

[syn. Nipporhynchus cadenati Golvan et Houin, 1964]

R. camerounensis Golvan, 1969

R. capensis Bray, 1974

R. carangis Yamaguti, 1939

R. chongmingnensis Huang, Zheng, Deng Fan et Ni, 1988

R. cololabis Laurs et McCauley, 1964

R. decapteri Parukhin et Kovalenko, 1976

R. ditrematis (Yamaguti, 1939) comb. $\mathbf{n}$.

[syns. Nipporhynchus ditrematis (Yamaguti, 1939) Ward, 1951; Protorhadinorhynchus ditrematis (Yamaguti, 1939) Ward, 1951]

R. dollfusi Gupta et Fatma, 1987

R. dorsoventrospinosus Amin, Heckmann et Ha, 2011

(Rhadinorhynchus dorsoventrospinosus sensu Al Ghamdi, 2013 is another species.)

R. dujardini Golvan, 1969

[syn. Rhadinorhynchus pristis sensu Cable et Linderoth, 1963]

R. echeneisi Gupta et Gupta, 1980

R. erumeii (Gupta et Fatima, 1981) Amin 1985

[syn. Nipporhynchus erumeii Gupta et Fatima, 1981]

R. ganapatii Chandra, Hanumantha-Rao et Shyamasundari, 1985

$\boldsymbol{R}$. hiansi Soota et Bhattacharya, 1981

R. japonicus Fujita, 1920 (nec japonicum)

R. johnstoni Golvan, 1969

[syn. Rhadinorhynchus pristis sensu Johnston et Edmonds, 1947]

R. keralensis Gupta et Fatma, 1987

R. laterospinosus Amin, Heckmann et Ha, 2011

R. lintoni Cable et Linderoth, 1963

[syn. Rhadinorhynchus pristis sensu Lühe, 1911]

R. ornatus Van Cleave, 1918

[syns. Rhadinorhynchus pristis sensu Linton, 1891; R. katsuwonis (Harada, 1928) Chandler 1934]

R. pelamysi Gupta et Gupta, 1980

R. plagioscionis Thatcher, 1980

R. plotosi Parukhin, 1985

R. polynemi Gupta et Lata, 1967

R. pristis (Rudolphi, 1802) (type species)

[syns. Echinorhynchus pristis Rudolphi, 1802; E. alosae Hermann, 1782; Rhadinorhynchus selkirki Van Cleave, 1921; R. subulatus Zeder, 1803]

R. salatrix Troncy et Vassiliades, 1973

R. selkirki Van Cleave, 1921

[syn. Rhadinorhynchus pristis sensu Chandler, 1934]

R. seriolae (Yamaguti, 1963) Golvan 1969

[syns. Nipporhynchus seriolae Yamaguti, 1963; Rhadinorhynchus pristis sensu Fukui et Morisita, 1937]

R. stunkardi Gupta et Fatma, 1987 (nec stunkardii)

R. trachuri (Harada, 1935) comb. n.

[syns. Nipporhynchus trachuri (Harada, 1935) Van Cleave et Lincicome, 1940; Rhadinorhynchus japonicus Fujita, 1920; R. selkirki Van Cleave, 1920 (fide Yamaguti 1939)]

R. trivandricus George et Nadakal, 1978

R. vancleavei Golvan, 1969

R. zhukovi Golvan, 1969

[syn. Rhadinorhynchus pristis sensu Zhukov, 1960]

GENUS Slendrorhynchus Amin et Sey, 1996

(moved from Diplosentidae)
SPECIES

S. breviclaviproboscis Amin et Sey, 1996 (type species)

SUBFAMILY Serrasentinae Petrochenko, 1956

GENUS Serrasentis Van Cleave, 1923

[syns. Echinorhynchus Müller, 1776, in part; Echinogaster Monticelli, 1905; Echinosoma Porta, 1907, in part; Lepidosoma Porta, 1908] (nec Serracentis)

(The placement of Serrasentis within the Echinorhynchida and not Polymorphida, based on analyses of nuclear $18 \mathrm{~S}$ rDNA sequences, was questioned by Verweyen et al. 2011.)

SPECIES

S. chauhani Datta, 1953

S. engraulisi Gupta et Gupta, 1980

S. fotedari Gupta et Fatma, 1980

S. golvani Gupta et Kumar, 1987

S. lamelliger (Diesing, 1854) Van Cleave 1923

[syns. Echinorhynchus lamelliger Diesing, 1854; Lepidosoma lamelliger (Diesing, 1854) Porta, 1908]

S. manazo Bilqees et Khan, 2005

S. mujibi Bilqees, 1972

S. nadakali George et Nadakal, 1978

S. niger Kahatoon et Bilqees, 2007

S. psenesi Gupta et Gupta, 1980

S. sagittifer (Linton, 1889) Van Cleave 1923 (type species)

[syns. Echinorhynchus sagittifer Linton, 1889; Echinogaster sagittifer (Linton, 1889) Porta, 1908; Serrasentis socialis (Leidy, 1851 nec 1858) Van Cleave, 1924; S. chauhani Datta, 1954; S. longa Tripathi, 1959; S. longiformis Bilqees, 1971; S. giganticus Bilqees, 1972; S. scomberomori Wang, 1981]

S. sauridae Surekha et Vijayalakshmi, 2006

S. sciaenus Bilqees, 1972 (nec scianis)

S. sidaroszakaio Tadros, Iskandar et Wassef, 1979

SUBFAMILY Serrasentoidinae Parukhin, 1982

GENUS Serrasentoides Parukhin, 1971

\section{SPECIES}

S. fistulariae Parukhin, 1971 (type species)

FAMILY Transvenidae Pichelin et Cribb, 2001

(The inclusion of species of Pararhadinorhynchus, or species relegated to this genus, to Transvenidae by Pichelin and Cribb 2001 is not accepted; see note under Sclerocollum above.)

GENUS Trajectura Pichelin et Cribb, 2001

\section{SPECIES}

T. ikedai (Machida, 1992) Pichelin et Cribb, 2001

[syn. Diplosentis ikedai Machida, 1992]

T. perinsolens Pichelin et Cribb, 2001 (type species)

GENUS Transvena Pichelin et Cribb, 2001

\section{SPECIES}

T. annulospinosa Pichelin et Cribb, 2001 (type species)

FAMILY Sauracanthorhynchidae Bursey, Goldberg et Kraus, 2007

GENUS Sauracanthorhynchus Bursey, Goldberg et Kraus, 2007

SPECIES

S. sphenomorphicola Bursey, Goldberg et Kraus, 2007 (type species)

ORDER HETERAMORPHIDA Amin et Ha, 2008

FAMILY Pyrirhynchidae Amin et $\mathrm{Ha}, 2008$

GENUS Pyrirhynchus Amin et Ha, 2008 
SPECIES

P. heterospinus Amin et Ha, 2008 (type species)

ORDER POLYMORPHIDA Petrochenko, 1956

FAMILY Centrorhynchidae Van Cleave, 1916 (Golvan 1960)

GENUS Centrorhynchus Lühe, 1911

[syns. Echinorhynchus Zoega in Müller, 1780, in part; Paradoxites Lindemann, 1865, preoccupied; Chentrosoma Porta, 1906, in part; Chentrorhynchus Neiva, Cunha et Travassos, 1914; Gordiorhynchus Meyer, 1931; Travassosina Witenberg, 1932]

(Golvan 1965 created the subgenus Maglacanthus, without formal diagnosis, for three species of Centrorhynchus in Madagascar, C. brumpti, C. brygooi - type species, and C. grassei, based on males with two cement glands and an additional double penile sphincter. A fourth species $C$. atheni Gupta et Fatma, 1983 was also described from India. We consider those two male traits as odd variations that do not deserve a special taxonomic recognition. Species of Centrorhynchus normally have 3-6 cement glands. Similarly, no subgeneric status was given to Neoechinorhynchus didelphis Amin, 2001, which has two uterine bells and unusual complex uterine system.)

\section{SPECIES}

C. acanthotrias (von Linstow, 1883) Petrochenko, 1958

C. albensis Rengaraju et Das, 1975

C. albidus Meyer, 1932

C. aluconis (Müller, 1780) Lühe 1911 (type species)

[syns. Echinorhynchus aluconis Müller, 1780; E. otidis Schrank, 1788; E. inequalis Rudolphi, 1808; E. appendiculatus Westrumb, 1821 ; E. soricis Rudolphi, 1819; Centrorhynchus appendiculatum Westrumb, 1821; C. olssoni Lundström, 1942]

C. amini Khan, Muti-ur-Rahman, Bilqees et Khatoon, 2010

C. amphibius Das, 1950

C. appendiculatus (Westrumb, 1821) Joyeux et Baer, 1937 (nec appendiculatum)

C. asturinus (Johnston, 1912) Johnston 1918

[syn. Gigantorhynchus asturinus Johnston, 1912]

C. atheni Gupta et Fatma, 1983

C. bancrofti (Johston et Best, 1943) Golvan (1956) 1958

[syn. Gordiorhynchus bancrofti Johston et Best, 1943]

C. batrachus Das, 1952

[syn. Centrorhynchus splendi Gupta et Gupta, 1970]

C. bazaleticus Kuraschvili, 1955

C. bengalensis Datta et Soota, 1954 (a Mediorhynchus?)

(fide Nama and Rothore 1984)

C. bethaniae George et Nadakal, 1987

C. bilqeesae Ghazi Khan et Noorun-Nisa, 2005

C. brama Rengaraju et Das, 1980 (nec bramae)

C. brevicaudatus Das, 1950

C. brumpti Golvan, 1965

C. brygooi Golvan, 1965

C. bubonis Yamaguti, 1939

C. buckleyi Gupta et Fatma, 1983

C. buteonis (Schrank, 1788) Kostylew, 1914

[syns. Echinorhynchus buteonis Schrank, 1788; E. caudatus Zeder, 1803; E. polyacanthoides Creplin, 1825; Centrorhynchus polyacanthus (Schrank, 1788) Kostylew, 1914; C. wedli (Sonsino, 1896) Porta 1909 (fide Porta 1909 but valid fide Dollfus 1951); Centrosoma buteonis in Porta 1910]

C. californicus Millzner, 1924

C. chabaudi Golvan, 1958

C. clitorideus (Meyer, 1931) Golvan 1958

[syn. Gordiorhynchus clitorideus Meyer, 1931] (nec clitorideum)

C. conspectus Van Cleave et Pratt, 1940

[syn. C. wardae Holloway, 1958]

C. crotophagicola Schmidt et Neiland, 1966

C. dimorphocephalus (Westrumb, 1821) Meyer 1932

[syns. Echinorhynchus dimorphocephalus Westrumb, 1821; Prosthorhynchus dimorphocephalus Westrumb, 1821]
C. dipsadis (von Linstow, 1888) Golvan, 1956

[syn. Echinorhynchus dipsadis von Linstow, 1888]

C. elongatus Yamaguti, 1935

C. falconis (Johnston et Best, 1943) Golvan, 1956

[syn. Gordiorhynchus falconis Johnston et Best, 1943]

C. fasciatus (Westrumb, 1821) Travassos, 1926

[syns. Echinorhynchus fasciatus Westrumb, 1821; E. motacillae

atricapillae Rudolphi, 1819]

C. fisheri Bhattacharya, 1999

C. freundi (Hartwich, 1953) Golvan, 1956

[syn. Gordiorhynchus freundi Hartwich, 1953] (nec Hartwick)

C. fukiensis Wang, 1966

C. galliardi Golvan, 1956

C. gendrei (Golvan, 1957) Golvan, 1960

[syn. Gordiorhynchus gendrei Golvan, 1957]

C. gibsoni Khan, Ghazi et Bilqees, 2002

C. giganteus Travassos, 1921 (nec 1919)

C. glaucidii Wang, 1966

C. globocaudatus (Zeder, 1800) Lühe, 1911

[syns. Echinorhynchus globocaudatus Zeder, 1800; E. tuba Rudolphi, 1802, in part]

C. golvani Anantaraman et Anantaraman, 1969

C. grassei Golvan, 1965

C. guira Lunaschi et Drago, 2010

C. guptai Golvan, 1969

[syn. Pomphorhynchus indicus Gupta et Lata, 1967 vide Amin et al.

2003; Centrorhynchus latai Golvan, 1994]

C. hagiangensis (Petrochenko et Fan, 1969) Amin 1985

[syn. Gordiorhynchus hagiangensis Petrochenko et Fan, 1969]

C. halcyonicola Smales, 2011

C. hartwichi Golvan, 1994

[syn. Centrorhynchus conspectus sensu Hartwich, 1956]

C. horridus (von Linstow, 1897) Meyer, 1932

[syns. Echinorhynchus horridus von Linstow, 1897; Prosthorhyn-

chus horridus (von Linstow, 1897) Travassos, 1926]

C. indicus Golvan, 1956

[syn. Centrorhynchus falconis Das 1950]

C. insularis Tubangui, 1933

C. itatsinis Fukui, 1929

[syns. Centrorhynchus miyanojyo Kanda, 1957; C. crocidurus Das, 1950: juvenile of C. itatsinis Fukui, 1929 (fide Yamaguti 1963)]

C. javanicus Rengaraju et Das, 1975 (nec javanicans)

C. knowlesi Datta et Soota, 1955

C. kuntzi Schmidt et Neiland, 1966

C. latai Golvan, 1994

[syn. Centrorhynchus indicus (Gupta et Lata, 1966) Gupta et Fatma, 1983]

C. leptorhynchus Meyer, 1932

C. lobianchii (Monticelli, 1887) Meyer, 1932

[syn. Sphaerirostris?] (fide Golvan 1994)

C. longicephalus Das, 1950

C. lukiensis Wang, 1966

C. lucknowensis Gupta et Fatma, 1983

C. mabuiae (von Linstow, 1908) Golvan, 1956

[syn. Echinorhynchus mabuiae von Linstow, 1908]

C. macrorchis Das, 1949

C. madagascariensis (Golvan, 1957) Golvan, 1960

[syn. Gordiorhynchus madagascariensis Golvan, 1957]

C. magnus Fukui, 1929

[syn. Centrorhynchus microchis Fukui, 1929]

C. mariauxi Smales, 2011

C. merulae Dollfus et Golvan, 1961

C. microcephalus (Bravo-Hollis, 1947) Golvan, 1956

[syn. Gordiorhynchus microcephalus Bravo-Hollis, 1947]

C. migrans Zuberi et Farooqi, 1974 (nec Farooq)

C. milvus Ward, 1956

C. mysentri Gupta et Fatma, 1983

C. narcissae Florescu, 1942 
C. nicaraguensis Schmidt et Neiland, 1966

C. nickoli Khan, Bilqees et Ghazi, 2001

C. ninnii (Stossich, 1891) Meyer, 1932

[syn. Echinorhynchus ninnii Stossich, 1891; Chentrosoma ninnii

Porta, 1908] (nec ninni)

C. olssoni Lundström, 1942

C. opimus Travassos, 1919

C. owli Bhattacharya, 1999

C. paramaryasis nom. nov.

[syn. Centrorhynchus maryasis sensu Gupta et Lata, 1967]

C. petrotschenkoi Kuraschvilli, 1955 (nec petrochenkoi)

C. polemaeti Troncy, 1970

C. polymorphus Travassos, 1926

(Centrorhynchus polymorphus sensu Hartwich, 1956 is another species fide Golvan 1994)

C. ptyasus Gupta, 1950

C. pycnonoti Wang, 1986

C. renardi (Lindemann, 1865) Van Cleave 1923

[syn. Paradoxites renardi Lindemann, 1865]

C. robustus Richardson et Nickol, 1995

C. sharmai (Gupta et Lata, 1966) Gupta et Fatma, 1983

C. sholapurensis Rengaraju et Das, 1975

C. sikkimensis Bhattacharya, 2003

C. simplex Meyer, 1932

C. sindhensis Khan, Khatoon et Bilqees, 2002

C. sinicus Wang, 1966

C. smyrnensis Bhattacharya, 2007

C. spilornae Schmidt et Kuntz, 1969

[syn. Centrorhynchus andamanensis Soota et Kansal, 1972) (fide Zafar and Farooqi 1981)]

C. spinosus (Kaiser, 1893) Van Cleave 1924

[syns. Echinorhynchus spinosus Kaiser, 1893; Centrorhynchus spinosus Van Cleave,1916]

C. tumidulus (Rudolphi, 1819) Neiva da Cunha et Travassos, 1914

[syns. Echinorhynchus tumidulus Rudolphi, 1819; E. caudatus Rudolphi, 1819; E. megacephalus Westrumb, 1821; Centrorhynchus microcervicanthus Das, 1950 [a juvenile of C. tumidulus]

(fide Yamaguti 1963) (nec microcerviacanthus); C. tumidulus Neiva da Cuhna et Travassos, 1914; Polyacanthorhynchus megalocephalus (Westrumb, 1821) Travassos, 1921] (nec tumidulum)

C. tyotensis Rengaraju et Das, 1977

C. undulatus Dollfus, 1950

GENUS Neolacunisoma Amin et Canaris, 1997

\section{SPECIES}

N. geraldschmidti Amin et Canaris, 1997 (type species)

GENUS Sphaerirostris Golvan, 1956

\section{SPECIES}

S. areolatus (Rudolphi, 1819) Golvan, 1956

[syns. Echinorhynchus areolatus Rudolphi, 1819; E. orioli Rudolphi, 1819; E. sigmoides Westrumb, 1821; Centrorhynchus areolatus (Rudolphi, 1819) Golvan, 1956]

S. batrachus (Das, 1952) comb. n.

[syns. Centrorhynchus batrachus Das, 1952; C. splendi (Gupta et Gupta, 1970) Golvan, 1994]

S. bipartitus (Soloviev, 1912) Golvan, 1956

[syn. Centrorhynchus bipartitus (Soloviev, 1912) Golvan, 1956]

S. cinctus (Rudolphi, 1819) Golvan, 1956

[syn. Centrorhynchus cinctus (Rudolphi, 1819) Meyer, 1932]

S. corvi (Fukui, 1929) Golvan, 1956

[syns. Centrorhynchus corvi (Fukui. 1929) Golvan, 1956; C. hargisi Gupta et Fatma, 1983]

S. dollfusi Golvan, 1994

[syn. Centrorhynchus picae sensu Dollfus, 1953]

S. embae Cholodkowski et Kostylew, 1916) Golvan, 1956

[syn. Centrorhynchus embae Cholodkowski et Kostylew, 1916]

S. erraticus (Chandler, 1925) Golvan, 1956

[syn. Centrorhynchus erraticus Chandler, 1925]
S. globuli (Nama et Rathore, 1984) Golvan, 1994

[syn. Centrorhynchus globuli Nama et Rathore, 1984]

S. lancea (Westrumb, 1821) Golvan, 1956

[syns. Echinorhynchus lancea Westrumb, 1821; E. vanelli Goeze,

1782; Centrorhynchus lancea (Westrumb, 1821) Skrjabin, 1913;

C. cinctus (Rudolphi, 1819) Meyer, 1932; C. embae Kholodkowski et Kostylew, 1916; C. scanensis Lundström, 1942 fide Van Cleave and Williams 1951]

S. lanceoides (Petrochenko, 1949) Golvan, 1956

[syn. Centrorhynchus lanceoides Petrochenko, 1949]

S. leguminosus (Soloviev, 1912) Golvan, 1956

[syn. Centrorhynchus leguminosus Soloviev, 1912]

S. lesiniformis (Molin, 1859) Golvan, 1994

[syns. Echinorhynchus lesiniformis Molin, 1859; Centrorhynchus lesiniformis (Molin, 1859) Meyer, 1932]

S. maryasis (Datta, 1933) Golvan 1956

[syn. Centrorhynchus maryasis Datta, 1933]

S. opimus (Travassos, 1919) Golvan, 1956

[syn. Centrorhynchus opimus Travassos, 1919]

S. physocoracis (Porta, 1913) Golvan 1956

[syns. Echinorhynchus physocoracis Porta, 1913; Centrorhynchus physocoracis Porta, 1913]

S. picae (Rudolphi, 1819) Golvan, 1958 (type species)

[syns. Echinorynchus picae Rudolphi, 1819; E. lobianchii Monticelli,

1887; E. teres Westrumb, 1821; Centrorhynchus picae (Rudolphi, 1819) Meyer, 1932]

(Two regions, $18 \mathrm{~S}$ and $28 \mathrm{~S}$ of nuclear ribosomal DNA, of S. picae were sequenced by Radwan 2012. The resulting phylogenetic trees suggest a paraphyletic arrangement of the two Palaeacanthocephala orders Echinorhynchida and Polymorphida.)

S. pinguis (Van Cleave, 1918) Golvan, 1956

[syns. Centrorhynchus pinguis Van Cleave, 1918; C. bipartitus Soloviev, 1912; C. corvi Fukui, 1929; C. skrjabini Petrochenko, 1949; Travassosina pinguis (Fukui, 1929) Witenberg, 1932]

S. reptans (Bhalerao, 1931) Golvan, 1956

[syn. Centrorhynchus reptans Bhalerao, 1931]

S. robustus (Datta, 1928) Golvan, 1994

[syn. Echinorhynchus robustus Datta, 1928]

S. saxicoloides (Nama et Rathore, 1984) Golvan, 1994

[syn. Centrorhynchus saxicoloides Nama et Rathore, 1984]

S. scanensis (Lundström, 1941-1942) Golvan, 1956

[syn. Centrorhynchus scanensis Lundström, 1942]

S. serpenticola (von Linstow, 1908) Golvan, 1956

[syn. Echinorhynchus serpenticola von Linstow, 1908]

S. skrjabini (Petrochenko, 1949) Golvan, 1956

[syns. Centrorhynchus corvi (Fukui, 1929) Golvan, 1956; C. skrjabini Petrochenko, 1949]

S. tenuicaudatus (Marotel, 1889) Amin, 1985

[syn. Echinorhynchus tenuicaudatus Marotel, 1889; Centrorhynchus tenuicaudatus (Marotel, 1889) Lühe, 1911]

S. turdi (Yamaguti, 1939) Golvan, 1956

[syns. Centrorhynchus turdi Yamaguti, 1939; Gordiorhynchus turdi (Yamaguti, 1939) Kamegai, 1963]

S. wertheimae Schmidt, 1975

FAMILY Plagiorhynchidae Golvan, 1960

SUBFAMILY Plagiorhynchinae Meyer, 1931

GENUS Paralueheia Saxena et Gupta, 2008

SPECIES

P. guptai Saxena et Gupta, 2008 (type species)

GENUS Plagiorhynchus Lühe, 1911

(The taxonomy of the genus Plagiorhynchus is based on the admittance of the subgeneric status outlined below as proposed by Schmidt and Kuntz 1966 and Amin et al. 1999.)

SUBGENUS Plagiorhynchus Lühe, 1911

SPECIES

P. (P.) allisonae Smales, 2002

P. (P.) charadrii (Yamaguti, 1939) Van Cleave, 1951

[syn. Prosthorhynchus charadrii Yamaguti, 1939]

(nec Golvan, 1956)

P. (P.) charadriicola (Dollfus, 1953) Golvan, 1956 
[syn. Prosthorhynchus charadriicola Dollfus, 1953]

P. (P.) crassicollis (Villot, 1875) Lühe, 1911 (type species)

[syns. Echinorhynchus crassicollis Villot, 1875; Plagiorhynchus inflatus Creplin, 1829; P. lanceolatus (von Linstow, 1876) Lühe, 1911]

P. (P.) freitasi Vicente, 1977

P. (P.) karachiensis Muti-Ur-Rahman, Khan, Khatoon et Bilqees, 2008

$\boldsymbol{P}$ (P.) lemnisalis Belopolskaya, 1958 (nec lemniscalis)

P. (P.) limnobaeni (Tubangui, 1933) Golvan, 1956

P. (P.) linearis (Westrumb, 1821) Golvan, 1956

[syns. Echinorhynchus linearis Westrumb, 1821 (nec lineare); Plagiorhynchus sternae Rudolphi, 1819; Prosthorhynchus linearis (Westrumb, 1821) Meyer, 1932]

P. (P.) menurae (Johnston, 1912) Golvan, 1956

[syn. Prosthorhynchus menurae Johnston, 1912]

P. (P.) odhneri Lundström, 1942

P. (P.) paulus Van Cleave et Williams, (1950) 1951 (nec paulum)

[syn. Prosthorhynchus paulus Van Cleave et Williams, 1951]

P. (P.) pigmentatum (de Marval, 1902) Meyer, 1933

[syn. Centrorhynchus cylindraceum of de Marval 1905]

$\boldsymbol{P}$. (P.) pittarum Tubangui, 1935

P. (P.) ponticus Lisitsyna, 1992

P. (P.) rectus (Linton, 1892) Van Cleave, 1918 (nec Sprehn, 1942) [syn. Prosthorhynchus rectus (Linton, 1892) Travassos, 1926]

$\boldsymbol{P}$ (P.) reticulatus (Westrumb, 1821) Golvan, 1956 (nec reticulatum) [syn. Prosthorhynchus reticulatus (Westrumb, 1821) Travassos, 1926]

P. (P.) rosai (Porta, 1910) Golvan, 1956

[syns. Echinorhynchus brumpti Blanc et Cauchemez, 1911; Plagiorhynchus brumpti Blanc et Cauchemez, 1911]

P. (P.) spiralis (Rudolphi, 1809) Golvan, 1956

[syn. Echinorhynchus spiralis Rudolphi, 1809]

P. (P.) totani (Porta, 1910) Golvan, 1956

[syns. Echinorhynchus totani Porta, 1910; Prosthorhynchus totani (Porta, 1910) Meyer, 1932] Plagiorhynchinae incertae sedis

P. (P.) rostratus (de Marval, 1902) (incertae sedis fide Golvan, 1994) (nec rostratum)

P. (P.) urichi (Cameron, 1936) Golvan, 1956

SUBGENUS Prosthorhynchus Kostylew, 1915

\section{SPECIES}

P. (Pr.) angrensis (Travassos, 1926) Schmidt et Kuntz, 1966 [syn. Prosthorhynchus angrense Travassos, 1928] (nec angrense)

P. (Pr.) asturi (Gupta et Lata, 1967) comb. n.

[syns. Rhadinorhynchus asturi Gupta et Lata, 1967; Prosthorhynchus asturi (Gupta et Lata, 1967) Golvan, 1994]

P. (Pr.) asymmetricus (Belopolskaya, 1983) comb. n.

[syn. Prosthorhynchus asymmetricus Belopolskaya, 1983]

P. (Pr.) bullocki Schmidt et Kuntz, 1966

P. (Pr.) cossyphicola Smales, 2010

P. (Pr.) cylindraceus (Goeze, 1782) Schmidt et Kuntz, 1966 (type species)

[syns. Echinorhynchus cylindraceus Goeze, 1782; E. pici Gmelin, 1791 fide Florescu and Ienistea 1984; E. merulae Gmelin, 1791 fide Florescu and Ienistea 1984; E. transversus (Rudolphi, 1819) Travassos 1926; E. obliquus Dujardin, 1845 fide Florescu and Ienistea 1984 Centrorhynchus cylindraceus (Goeze 1782) Kostylew, 1914: C. fasciatus (Westrumb, 1821) Travassos, 1926 fide de Marval 1905; C. rostratus de Marval, 1902 fide Florescu and Ienistea 1984; Prosthorhynchus rosai (Porta, 1910) Meyer, 1932; Prosthorhynchus rostratus (de Marval, 1902) Meyer, 1932; Plagiorhynchus formosus Van Cleave, 1918 fide Amin et al. 1999; Plagiorhynchus taiwanensis Schmidt et Kuntz, 1966 fide Amin et al. 1999]

P. (Pr.) deysarkari Bhattacharya, 2002

P. $(\boldsymbol{P r}$.) digiticephalus Amin, Ha et Heckmann, 2008

P. (Pr.) gallinagi (Schachtachtinskaja, 1953) Schmidt et Kuntz, 1966 [syn. Prosthorhynchus gallinagi Schachtachtinskaja, 1953]

P. (Pr.) genitopapillatus (Lundström, 1942) Amin, 1985 [syn. Prosthorhynchus genitopapillatus Lundström, 1942] P. (Pr.) golvani Schmidt et Kuntz, 1966

P. (Pr.) gracilis (Petrochenko, 1958) Schmidt et Kuntz, 1966

P. (Pr.) kuntzi (Gupta et Fatma, 1988) Bhattacharya, 2007
P. (Pr.) limnobaeni (Tubangui, 1933) Golvan, 1956 [syn. Prosthorhynchus limnobaeni Tubangui, 1933]

P. (Pr.) longirostris (Travassos, 1926) Amin 1985 [syn. Prosthorhynchus longirostris Travassos, 1926]

P. (Pr.) luehei (Travassos, 1916) comb. n.

[syn. Prosthorhynchus luehei Travassos, 1916]

P. (Pr.) malayensis (Tubangui, 1935) Schmidt et Kuntz, 1966 (nec malayense)

[syn. Oligoterorhynchus malayensis Tubangui, 1935]

P. (Pr.) megareceptaclis Amin, Ha et Heckmann, 2008

P. (Pr.) nicobarensis (Soota et Kansal, 1970) Zafar et Farooqui, 1981

[syn. Prosthorhynchus nicobarensis Soota et Kansal, 1970]

P. (Pr.) ogatai (Fukui et Morisita, 1936) Schmidt et Kuntz, 1966 [syn. Porrochis ogatai Fukui et Morisita, 1936]

P. (Pr.) pittarum (Tubangui, 1935) Schmidt et Kuntz, 1966

[syn. Prosthorhynchus pittarum Tubangui, 1935]

P. (Pr.) reticulatus (Westrumb, 1821) Golvan, 1956

[syn. Echinorhynchus reticulatus Westrumb, 1821]

P. (Pr.) rheae (de Marval, 1902) Schmidt et Kuntz, 1966

[syn. Echinorhynchus rheae de Marval, 1902; E. rostratum de Marval, 1902]

P. (Pr.) rossicus (Kostylew, 1915) Schmidt et Kuntz, 1966

P. (Pr.) russelli (Tadros, 1970) Golvan 1994

[syn. Plagiorhynchus russelli Tadros, 1970]

P. (Pr.) schmidti Golvan, 1994

[syns. Echinorhynchus rectus Linton, 1892; Prosthorhynchus rectus Sprehn, 1942 (nec Linton, 1892)]

P. (Pr.) scolopacidis (Kostylew, 1915) Schmidt et Kuntz, 1966 [syn. Prosthorhynchus scolopacidis Kostylew, 1915]

P. (Pr.) transversus (Rudolphi, 1819) Travassos, 1926

$\boldsymbol{P}$. (Pr.) varispinus (Wang, 1966) comb. n.

[syn. Prosthorhynchus varispinus Wang, 1966]

SUBFAMILY Porrorchinae Golvan, 1956

GENUS Lueheia Travassos, 1919

[syn. Furcata Werby, 1938]

SPECIES

L. adlueheia (Werby, 1938) Van Cleave, 1942

[syn. Furcata adlueheia Werby, 1938]

L. cajabambensis Machado Filho et Ibanez, 1967

L. inscripta (Westrumb, 1821) Travassos, 1920

[syn. Echinorhynchus inscripta Westrumb, 1821]

L. karachiensis Khan, Bilqees et Muti-ur-Rahman, 2005

L. lueheia Travassos, 1919 (type species) (nec luehei)

GENUS Oligoterorhynchus Monticelli, 1914

\section{SPECIES}

O. campylurus (Nitzsch, 1857) Monticelli, 1914 (type species)

[syn. Echinorhynchus campylurus Nitzsch, 1857]

GENUS Owilfordia Schmidt et Kuntz, 1967

SPECIES

O. olseni Schmidt et Kuntz, 1967 (type species)

O. schmidti Gupta et Fatma, 1988

O. teliger (Van Cleave, 1949) Schmidt et Kuntz, 1967

[syn. Porrochis teliger Van Cleave, 1949]

GENUS Porrorchis Fukui, 1929

[syn. Pseudoporrochis Joyeux et Baer, 1935]

SPECIES

P. aruensis Smales, 2010

P. bazae (Southwell et Macfie, 1925) Schmidt et Kuntz, 1967

[syns. Echinorhynchus bazae Southwell et Macfie, 1925; Prosthorhynchus bazae (Southwell et Macfie, 1925) Travassos, 1926; Pseudoporrorchis bazae (Southwell et Macfie, 1925) Petrochenko, 1958]

P. brevicanthus (Das, 1949) Golvan, 1994

[syn. Centrorhynchus brevicanthus Das, 1949-1950]

P. centropi (Porta, 1910) Schmidt et Kuntz, 1967 
[syns. Echinorhynchus centropi Porta, 1910; E. centropi (Porta, 1910) Joyeux et Baer, 1935]

P. chauhani Gupta et Fatma, 1986

P. crocidurai Gupta et Fatma, 1986

P. elongatus Fukui, 1929 (type species)

P. heckmanni Bilqees, Khan, Khatoon et Khatoon, 2007

P. herpistis Bhattacharya, 2007

P. houdemeri (Joyeux et Baer, 1935) Schmidt et Kuntz, 1967

[syn. Pseudoporrochis houdemeri Joyeux et Baer, 1935]

P. hydromuris (Edmonds, 1957) Schmidt et Kuntz, 1967

[syn. Pseudoporrochis hydromuris Edmonds, 1957]

P. hylae (Johnston, 1914) Schmidt et Kuntz, 1967

[syns. Echinorhynchus hylae Johnston, 1914; E. bulbocaudata Southwell et McFie, 1925; E. centropusi (Tubangui, 1933) Petrochenko, 1958; Gordiorhynchus hylae (Johnston, 1914) Johnston et Edmonds, 1948; Prosthorhynchus bulbocaudatus (Southwell et McFie, 1925) Travassos, 1926; Pseudoporrorchis bulbocaudatus (Southwell et MacFie, 1925) Joyeux et Baer, 1935; P centropusi (Tubangui, 1933) Joyeux et Baer, 1935; Centrorhynchus hylae (Johnston, 1914) Schmidt et Kuntz, 1967]

P. indicus (Das, 1957) Schmidt et Kuntz, 1967

[syn. Pseudoporrochis indicus Das, 1957]

P. jonesae Muti-ur-Rahman, Khan, Khatoon et Bilqees, 2010

P. keralensis George et Nadakal, 1984

P. kinsellai Lisitsyna, Tkach et Bush, 2012

P. leibyi Schmidt et Kuntz, 1967

P. maxvachoni (Golvan et Brygoo, 1965) Schmidt et Kuntz, 1967 [syn. Pseudoporrochis maxvachoni Golvan et Brygoo, 1965]

P. nickoli Salgado-Maldonado et Cruz-Reyes, 2002

$\boldsymbol{P}$ oti Yamaguti, 1939 (nec goti)

P. rotundatus (von Linstow, 1897) Schmidt et Kuntz, 1967

[syns. Echinorhynchus rotundatus von Linstow, 1897; Pseudoporrorchis rotundatus (von Linstow, 1897) Joyeux et Baer, 1935] (nec rotundus)

P. tyto Amin, Ha et Heckmann, 2008

GENUS Pseudogordiorhynchus Golvan, 1957

SPECIES

P. antonmeyeri Golvan, 1957 (type species)

GENUS Pseudolueheia Schmidt et Kuntz, 1967

\section{SPECIES}

P. arunachalensis Bhattacharya, 2007

P. boreotis (Van Cleave et Williams, 1951) Schmidt et Kuntz, 1967 [syn. Lueheia boreotis Van Cleave et Williams, 1951]

P. korathai Gupta et Fatma, 1988

P. pittae Schmidt et Kuntz, 1967 (type species)

P. tongsoni Salcedo et Celis, 2007

SUBFAMILY Sphaerechinorhynchinae Golvan, 1956

GENUS Sphaerechinorhynchus Johnston, 1929

\section{SPECIES}

S. macropisthospinus Amin, Wongsawad, Marayong, Saehoong, Suwattanacoupt et Sey, 1998

S. maximesospinus Amin, Ha et Heckmann, 2008

S. ophiograndis Bolette, 1997

S. rotundocapitatus (Johnston, 1912) Johnston et Deland, 1929 (type species)

[syn. Echinorhynchus rotundocapitatus Johnston, 1912]

S. serpenticola Schmidt et Kuntz, 1966

FAMILY Polymorphidae Meyer, 1931

[syn. Filicollidae Petrochenko, 1956]

GENUS Andracantha Schmidt, 1975

\section{SPECIES}

A. baylisi (Zdzitowiecki, 1986) Zdzitowiecki, 1989

[syn. Corynosoma baylisi Zdzitowiecki, 1986]

A. clavata (Goss, 1941) Zdzitowiecki 1989 [syn. Corynosoma clavatum Goss, 1941]

A. gravida (Alegret, 1941) Schmidt 1975 (type species)

[syn. Corynosoma gravida Alegret, 1941]

A. mergi (Lundström, 1941) Schmidt, 1975

[syns. Corynosoma mergi Lundström, 1941; Hemiechinosoma mergi (Lundström, 1941) Petrochenko et Smogorjevskaya, 1962]

A. phalacrocoracis (Yamaguti, 1939) Schmidt, 1975

[syn. Corynosoma phalacrocoracis Yamaguti, 1939]

A. tandemtesticulata Monteiro, Amato et Amato, 2006

A. tunitae (Weiss, 1914) Zdzitowiecki 1989

[syn. Corynosoma tunitae Weiss, 1914]

GENUS Ardeirhynchus Dimitrova et Georgiev, 1994

SPECIES

A. spiralis (Rudolphi, 1809) Dimitrova et Georgiev, 1994

(type species)

[syns. Echinorhynchus spiralis Rudolphi, 1809; Prosthorhynchus spiralis (Rudolphi, 1809) Meyer, 1932; Plagiorhynchus spiralis (Rudolphi, 1809) Golvan, 1956]

GENUS Arhythmorhynchus Lühe, 1911

[syn. Skrjabinorhynchus Petrochenko, 1956]

\section{SPECIES}

A. capellae (Yamaguti, 1935) Schmidt 1973

[syns. Polymorphus capellae Yamaguti, 1935; Skrjabinorhynchus capellae (Yamaguti, 1935) Van Cleave et Rausch, 1950] (nec Schmidt, 1973)

A. comptus Van Cleave et Rausch, 1950

A. distinctus Baer, 1956

A. eroliae (Yamaguti, 1939) Schmidt, 1973

[syns. Polymorphus eroliae Yamaguti, 1939; Skrjabinorhynchus eriolae (Yamaguti, 1939) Petrochenko, 1956]

A. frassoni (Molin, 1858) Lühe, 1911 (type species)

[syns. Echinorhynchus frassoni Molin, 1858; E. roseus Molin, 1858; E. rubicundus Molin, 1858; Arhythmorhynchus macrourus (Bremser in Westrumb, 1921) (fide Meyer 1932) or species inquirenda fide Khokhlova 1975; A roseus (Molin, 1858) Meyer, 1932 (fide Golvan 1956, fide Petrochenko 1958); A. globicollis Creplin, 1829; A. rubicundus (Molin, 1859) Meyer, 1932 juvenile (fide Golvan 1956)]

A. frontospinosus (Tubangui, 1935) Yamaguti, 1963

[syn. Polymorphus frontospinosus Tubangui, 1935]

A. jeffreyi Schmidt, 1973

[syn. Arhythmorhynchus capellae Schmidt, 1963]

A. johnstoni Golvan, 1960

[syn. Arhythmorhynchus frassoni sensu Johnston et Edmonds, 1951]

A. limosae Edmonds, 1971

A. longicollis (Villot, 1875) Lühe, 1912

[syns. Echinorhynchis longicollis Villot, 1875; E. invaginabilis von Linstow, 1902; E. macrourus Bremser, 1821; Arhythmorhynchus anser Florescu, 1941; A. invaginabilis (von Linstow, 1902) Lühe, 1912 (fide Golvan 1956, Khokhlova 1975) (nec invaginavilis); A. roseus (Molin, 1858) Meyer, 1932 (fide Golvan 1956, fide Petrochenko 1958)]

A. petrotchenkoi (Schmidt, 1969) Atrashkevich, 1979

[syn. Polymorphus petrochenkoi Schmidt, 1969] (nec petroschenkoi)

A. plicatus (von Linstow, 1883) Meyer, 1932

[syn. Echinorhynchus plicatus von Linstow,1883]

A. pumiliorostris Van Cleave, 1916

(nec plumirostris, nec pumilliorostris)

A. roseus (Molin, 1858) Meyer, 1932 (valid fide Khokhlova 1975)

A. rubicundus (Molin, 1859) Meyer, 1932

(valid fide Khokhlova 1975)

A. siluricola Dollfus, 1929

[syn. Echinorhynchus sensu lato] (fide Golvan 1969)

A. suecicus Lundström, 1942

A. teres Van Cleave, 1920

[syn. Arhythmorhynchus sachalinense Krotov et Petrochenko, 1958] (fide Khokhlova 1975)

A. tigrinus Moghe et Das, 1953

A. trichocephalus (Leuckart, 1876) Lühe, 1912

[syn. Echinorhynchus trichocephalus Leuckart, 1876]

A. tringi Gubanov, 1952 
A. turbidus (Van Cleave, 1937) Golvan, 1994 [syn. Corynosoma turbidum Van Cleave, 1937]

A. uncinatus (Kaiser, 1893) Lühe, 1912

[syn. Echinorhynchus uncinatus Kaiser, 1893]

A. villoti Golvan, 1994

[syn. Echinorhynchus longicollis Villot, 1875]

A. xeni Atrashkevich, 1978

GENUS Bolbosoma Porta, 1908

[syns. Echinorhynchus Zoega in Müller, 1776, in part; Bolborhynchus Porta, 1906]

\section{SPECIES}

B. australis Skrjabin, 1972

B. balaenae (Gmelin, 1790) Porta, 1908

[syns. Echinorhynchus balaenae Gmelin, 1790; E. lendix Phipps, 1774; E. porrigens (Rudolphi, 1814) Porta, 1908; Bolbosoma porrigens (Rudolphi, 1814) Porta, 1908] (fide Van Cleave 1953)

B. brevicolle (Malm, 1867) Porta, 1908

[syns. Echinorhynchus brevicollis Malm, 1867; Bolborhynchus brevicolle (Malm, 1867) Porta, 1906]

B. caenoforme (Heitz, 1920) Meyer, 1932 (nec Heitz, 1917) (may be Corynosoma fide Golvan 1960, fide Yamaguti 1963)

B. capitatum (von Linstow, 1880) Porta, 1908

[syns. Echinorhynchus capitatum von Linstow, 1880; Bolbosoma physeteris Gubanov, 1952 (fide Amin and Margolis 1998)]

B. hamiltoni Baylis, 1929

B. heteracanthe (Heitz, 1920) Meyer, 1932

B. nipponicum Yamaguti, 1939

[syn. B. bobrovoi Krotov et Delyamure, 1952 (nec bobrovi, nec Delamure, nec Delamare)]

B. scomberomori Wang, 1980

B. tuberculata Skrjabin, 1970

B. turbinella (Diesing, 1851) Porta 1908 (type species)

[syns. Echinorhynchus turbinella Diesing, 1851; Bolborhynchus turbinella (Diesing, 1851) Porta, 1906; Bolbosoma balaenocephalus Owen, 1803; B. ruber Collet, 1886] (B. turbinella sensu Leiper et Atkinson, 1915 is another species) (fide Meyer 1932)

B. vasculosum (Rudolphi, 1819) Porta, 1908

[syns. Echinorhynchus vasculosum Rudolphi, 1819; Bolbosoma annulatus Molin, 1858; B. aurantiacus Risso, 1826; B. pellucidus Leukart, 1828; B. serrani Linton, 1888; B. thunni Harada, 1935 (fide Petrochenko 1958)]

GENUS Corynosoma Lühe, 1904 (fide Van Cleave 1945)

[syns. Chentrosoma Monticelli, 1905; Centrosoma Lühe 1912; Coryusoma Railliet et Henry, 1907 (misprint); Echinosoma Porta, 1907]

(Aznar et al. 2006 split Corynosoma to two genera, Corynosoma for marine species and Pseudocorynosoma for freshwater species, based on anatomical, ecological and phylogenetic divergences. This arrangement is accepted herein. García-Varela et al. 2009 further demonstrated that Pseudocorynosoma is an independent lineage that does not share a common ancestry with Corynosoma or Andracantha.)

\section{SPECIES}

(marine species - see note above)

C. alaskensis Golvan, 1959 (nec alaskaensis)

C. australe Johnston, 1937

[syn. Corynosoma otariae Morini et Boero, 1961]

C. baylisi Zdzitowiecki, 1986

C. bullosum (von Linstow, 1892) Railliet et Henry, 1907

[syns. Echinorhynchus bullosum von Linstow, 1892; Corynosoma arctocephali Zdzitowiecki, 1984; C. mirabilis Skrjabin, 1966 (fide Zdzitowiecki 1986); C. singularis Skrjabin et Nikolski, 1971, in part]

C. cameroni Van Cleave, 1953

[syn. Corynosoma strumosum sensu Lyster, 1940]

C. caspicum Golvan et Mokhayer, 1973

C. cetaceum (Johnston et Best, 1942) Aznar, Bush et Raga, 2002 [syns. Corynosoma semerme sensu Cordero, 1933? (fide Schmidt and Dailey 1971); Polymorphus cetaceum (Johnston et Best, 1942) Schmidt et Dailey 1971; P. arctocephali Smales, 1986 (nec arctocephalus)]
C. constrictum Van Cleave, 1918

[syn. Corynosoma bipallatum Schmidt, 1965]

C. curilensis Gubanov, 1942 (nec kurilense, nec curiliensis)]

C. enhydri Morozov, 1940

[syns. Corynosoma enhydris Afanasev, 1941; C. macrosomum Neiland, 1962] (fide Jellison and Neiland 1965)

C. eperlani (von Linstow, 1884) Petrochenko, 1958

C. erignathi Stryukov, 2000

C. evae Zdzitowiecki, 1984

C. falcatum Van Cleave, 1953

C. gibsoni Zdzitowiecki, 1986

C. hadweni Van Cleave, 1953 (nec hadveni)

[syn. Corynosoma semerme sensu Lyster, 1940, in part] (fide Van Cleave 1953)

C. hamanni (von Linstow, 1892) Railliet et Henry, 1907 (nec Leiper et Atkinson, 1915)

[syns. Echinorhynchus hamanni von Linstow, 1892; E. antarcticum Rennie, (1906) 1907; Corynosoma antarticum (Rennie, 1906) Johnston et Best, 1937 (nec Leiper et Atkinson, 1918); C. pacifica Nikolskii, 1974; C. sipho Railliet et Henry, 1907]

C. hannae Zdzitowiecki, 1984

C. longilemniscatus Machado Filho, 1961

[syn. Corynosoma peposacae sensu Travassos, 1925]

C. macrosomum Neiland, 1962

C. magdaleni Montreuil, 1958 (nec magdaleini)

C. mandarinca Oschmarin, 1963

C. obtuscens Lincicome, 1943

C. osmeri Fujita, 1921

[syn. Corynosoma ambispierinum Harada, 1935]

C. otariae Morini et Boero, 1960

[syn. Corynosoma australe Johnston, 1937] (fide Zdzitowiecki 1989)

C. pseudohamanni Zdzitowiecki, 1984

C. pyriforme (Bremse, 1824) Meyer, 1932

[syn. Echinorhynchus pyriforme Bremser, 1824]

(may be a Polymorphus (fide Machalska 1981)

C. rauschi Golvan, 1959

C. reductum (von Linstow, 1905) Railliet et Henry, 1907

[syn. Echinorhynchus reductus von Linstow, 1905]

C. semerme (Forssell, 1904) Lühe, 1905 (nec Frossell)

[syns. Echinorhynchus semermis Forssell, 1904; Corynosoma gibber

(Olsson, 1894) Lühe, 1911] (C. semerme sensu Fukui, 1929)

C. septentrionalis Treshchev, 1966

C. seropedicus Machado Filho, 1970

[syn. C. clemente Giovannoni et Fernandes, 1965 - see Amin 1985] (nec clementi)

C. shackletoni Zdzitowiecki, 1978

C. similis Neiland, 1962

C. stanleyi Smales, 1986

C. strumosum (Rudolphi, 1802) Lühe, 1904 (type species)

[syns. Echinorhynchus strumosum Rudolphi, 1802; Corynosoma ambispinigerum Harada, 1935; C. carchariae Linton, 1891; C. gibber Olsson, 1893; C. gibbosus Rudolphi, 1809, in part; C. hystrix Bremser, 1824; C. incrassatus Linton, 1891; C. osmeri Fujita, 1921; C. striatus Villot, 1875; C. ventricosus Rudolphi, 1809] (Corynosoma strumosum sensu Dogiel et Bychowsky, 1938, Hartwich, 1956, and Dollfus, 1962 are other species, fide Golvan 1994)

C. sudsuche Belopolskaya, 1958

C. tunita Weiss, 1914

[syn. Corynosoma hystrix tunitae Weiss, 1914]

C. turbidum Van Cleave, 1937

C. validum Van Cleave, 1953

[syn. Corynosoma semerme sensu Lyster, 1940, in part (fide Van Cleave 1953)]

C. ventronudum Skrjabin, 1959

C. villosum Van Cleave, 1953 (nec viliosum)

C. wegeneri Heinze, 1934

[syn. Corynosoma hadweni Van Cleave, 1953] (nec hadveni) (fide Margolis 1955)]

GENUS Diplospinifer Fukui, 1929 
SPECIES

D. serpenticola Fukui, 1929 (type species)

GENUS Filicollis Lühe, 1911

SPECIES

F. anatis (Schank, 1788) Lühe 1911 (type species)

[syns. Echinorhynchus anatis Schank, 1788; E. filicollis Rudolphi,

1809; E. laevis von Linstow, 1905; E. polymorphus Bremser, 1824]

F. trophimenkoi Atrashkevich, 1982

GENUS Ibirhynchus García-Valera, Pérez-Ponce de León, Aznar et Nadler, 2011

\section{SPECIES}

I. dimorpha (Schmidt, 1973) García-Valera, Pérez-Ponce de León,

Aznar et Nadler, 2011 (type species)

[syn. Southwellina dimorpha Schmidt, 1973]

(The erection of Ibirhynchus by García-Valera et al. 2011 was based

on analyses of sequences of two nuclear genes.)

GENUS Polymorphus Lühe, 1911

[syns. Hexaglandula Petrochenko, 1950 (fide Amin 1992); Subcorynosoma Khokhlova, 1967 (fide Amin 1992); Subfilicollis Khokhlova, 1967]

(Amin's 1992 revision of Polymorphus recognised two subgenera, Polymorphus Lühe, 1911 and Profilicollis Meyer, 1931. The elevation of these two subgenera to the generic level has been controversial, e.g. Nickol et al. 1999 supports it based on intermediate host affinities but García-Varela and Pérez-Ponce de León (2008) disagree based on sequences of the cox 1 gene. The elevation to generic level is provisionally admitted herein. The above synonymies, however, stand even though the synonymy of Hexaglandula and Polymorphus remains controversial; see Nickol et al. 1999 and García-Varela and Pérez-Ponce de León 2008.)

SPECIES

P. actuganensis Petrochenko, 1949

P. acutis Van Cleave et Starrett, 1940

P. arctocephali Smales, 1986 (nec arctocephalus)

$\boldsymbol{P}$ ariusus (Bilqees, 1971) comb. $\mathbf{n}$

[syn. Hexaglandula ariusus Bilqees, 1971]

P. biziurae Johnston et Edmonds, 1948

P. boschadis (Schrank, 1788) Railliet, 1919 (type species fide Yamaguti 1963)

[syn. Polymorphus phippsi Kostylew, 1922]

P. brevis (Van Cleave, 1916)

[syn. Arhythmorhynchus brevis Van Cleave, 1916 (fide Amin 1992)] (Using sequences of the cox 1 and rRNA genes and morphometric analysis, Alcántar-Escalera et al. 2013 established that cystacanths from freshwater fishes in Central Mexico and adults from fish-eating birds belong to $P$. brevis.

P. chongqingensis Liu, Zhang et Zhang, 1990

P. cincli Belopolskaya, 1959

P. contortus (Bremser, 1821) Travassos, 1926

[syns. Echinorhynchus contortus Bremser, 1821; E. collurionis Rudolphi, 1819]

(may not be Polymorphus, fide Golvan 1994)

P. corynoides (Skrjabin, 1913) comb. n.

[syn. Subcorynosoma corynoides Skrjanin, 1913]

P. corynosoma (Travassos, 1915) comb. $\mathbf{n}$.

[syn. Hexaglandula corynosoma (Travassos, 1915) Petrochenko, 1958]

P. crassus Van Cleave, 1924

P. cucullatus Van Cleave et Starret, 1940

P. diploinflatus Lundström, 1942

P. fatimaae Khan, Dharejo, Birmani et Bilqees, 2008

P. fulicai Birmani, Dharejo et Khan, 2011

P. gavii (Kokhlova, 1965) comb. n.

[syn. Subfilicollis gavii Khokhlova, 1965]

P. inermis (Travassos, 1923) comb. $\mathbf{n}$.

[syn. Hexaglandula inermis (Travassos, 1923) Petrochenko, 1958]

P. karachiensis (Bilqees, 1971) comb. n.

[syn. Hexaglandula karachiensis Bilqees, 1971]
P. kostylewi Petrochenko, 1949

P. magnus Skrjabin, 1913

P. marchii (Porta, 1910) Meyer, 1932

[syn. Corynosoma marchii, Porta, 1910 (fide Meyer 1932)]

P. marilis Van Cleave, 1939

P. mathevossianae Petrochenko, 1949 (nec mathevossiaenae)

P. meyeri Lundström, 1942

P. miniatus (von Linstow, 1896) Travassos, 1926

[syn. Echinorhynchus miniatus von Linstow, 1896]

P. minutus (Goeze, 1782) Lühe, 1911 (type species)

[syns. Echinorhynchus minutus Goeze, 1782; E. boschadis Schrank, 1788; E anatis Gmelin, 1791; E collaris Schrank, 1792; Polymorphus boschadis (Schrank, 1788) Railliet, 1919; P. magnus Skrjabin, 1913] (fide Amin 1992)

P. mohiuddini Muti ur-Rahman, Khan, Bilqees et Khatoon, 2008

P. mutabilis (Rudolphi, 1819) Petrochenko, 1950

[syn. Echinorhynchus mutabilis Rudolphi, 1819; Hexaglandula mutabilis Rudolphi, 1819]

P. nickoli Khan et Bilqees, 1998

P. obtusus Van Cleave, 1918

P. paradoxus Connel et Corner, 1957

P. paucihamatus (Heinze, 1936) comb. n. (nec paucihamata)

[syn. Hexaglandula paucihamatus (Heinze, 1938) Petrochenko, 1958]

P. phippsi (Kostylew, 1922) comb. n.

[syns. Echinorhynchus borealis Gmelin, 1791; E. mollissimae Rudolphi, 1809; Subfilicollis phippsi (Kostylew, 1922) Khokhlova, 1967; Polymorphus boschadis Ralliet, 1919, fide Van Cleave and Rausch 1951]

P. piriformis (Bremser, 1811 in Rudolphi, 1819) Machalska, 1981

P. pupa (von Linstow, 1905) Kostylew, 1922

(fide Khokhlova 1986; fide Amin 1992) (species inquirenda fide Van Cleave and Rausch 1951, fide Yamaguti 1963)

[syn. Echinorhynchus pupa von Linstow, 1905; Filicollis pupa (von Linstow, 1905) Travassos, 1926; Plagiorhynchus (Prosthorhynchus) pupa (von Linstow, 1905) Meyer, 1931]

P. sichuanensis Wang et Zhang, 1987

P. sindensis Khan, Ghazi et Bilqees, 2002

P. spindlatus Amin et Heckmann, 1991

P. striatus (Goeze, 1782) Lühe, 1911

[syns. Echinorhynchus striatus Goeze, 1782; E. ardeae Gmelin, 1789]

P. strumosoides (Lundström, 1942) comb. n.

[syn. Subcorynosoma strumosoides Lundström, 1942]

P. swartzi Schmidt, 1965

P. trochus Van Cleave, 1945

GENUS Profilicollis Meyer, 1931

[syns. Falsifilicollis Webster, 1948; Profilicollis Petrochenko, 1956]

\section{SPECIES}

P. altmani (Perry, 1942) Van Cleave, 1947

[syns. Filcollis altmani Perry, 1942; Parafilicollis altmani (Perry, 1942) Petrochenko, 1956; Polymorphus bullocki Mateo, Cordova et Guzman, 1982; Profilicollis kenti (Van Cleave, 1947) Khokhlova, 1974; Polymorphus kenti Van Cleave, 1947; Parafilicollis kenti (Van Cleave, 1947) Petrochenko, 1956; Falsificollis kenti (Van Cleave, 1947) Yamaguti, 1963 fide Nickol et al. 2002; Filicollis sphaerocephalus sensu Harrington et Pillbury, 1938 fide Tantaleán et al. 2005; Profilicollis texensis (Webster, 1948) Khokhlova, 1974; Polymorphus (Falsificollis) texensis (Webster, 1948) Yamaguti, 1963 fide Nickol et al. 2002]

P. antarcticus Zdzitowiecki, 1985

P. arcticus (Van Cleave, 1920) Meyer, 1932

[syn. Filcollis arcticus Van Cleave, 1920]

P. botulus (Van Cleave, 1916) Witenberg, 1932 (type species) [syn. Filicollis botulus Van Cleave, 1916]

P. chasmagnathi (Holcman-Spector, Mane-Garzon et Dei-Cas, 1978) Amin, 1992

[syn. Falsifilicollis chasmagnathi Holcman-Spector, Mane-Garzon et Dei-Cas, 1978]

P. formosus (Schmidt et Kuntz, 1967) Khokhlova, 1974 
[syn. Polymorphus formosus Schmidt et Kuntz, 1967]

P. major (Lundström, 1942) Khokhlova, 1974

[syns. Polymorphus major Lundström, 1942; Parafilicollis major (Lundström, 1942) Petrochenko, 1956; Filicollis major Lundström,1942; Falsificollis major (Lundström, 1942) Yamaguti, 1963]

P. novaezelandensis Brockerhoff et Smales, 2002

P. sphaerocephalus (Bremser in Rudolphi, 1819) Van Cleave, 1947

[syns. Echinorhynchus sphaerocephalus Bremser in Rudolphi, 1819; Filicollis sphaerocephalus (Bremser in Rudolphi, 1819) Travassos, 1928; Parafilicollis sphaerocephalus (Bremser in Rudolphi, 1819) Petrochenko, 1956; Falsifilicollis sphaerocephalus (Bremser in Rudolphi, 1819) Yamaguti, 1963]

GENUS Pseudocorynosoma Aznar, Pérez-Ponce de León et Raga, 2006

(freshwater species of former species of Corynosoma)

\section{SPECIES}

P. anatarium (Van Cleave, 1945) Aznar, Pérez-Ponce de León et Raga, 2006

[syn. Corynosoma anatarium Van Cleave, 1945]

P. constrictum (Van Cleave, 1918) Aznar, Pérez-Ponce de León et Raga, 2006 (type species)

[syns. Echinorhynchus striatus Goeze sensu Linton, 1892; Corynosoma constrictum Van Cleave, 1918; C. bipapillum Schmidt, 1965]

P. enrietti (Molfie et Freitas-Fernandes, 1953) Aznar, Pérez-Ponce

de León et Raga, 2006

[syns. Corynosoma enrietti Molfie et Freitas-Fernandes, 1953; C. molfifernandesi Machado Filho, 1962 fide Golvan 1994]

P. iheringi (Machado Filho, 1961) Aznar, Pérez-Ponce de León et Raga, 2006

[syn. Corynosoma iheringi Machado Filho, 1961]

P. peposacae (Porta, 1914) Aznar, Pérez-Ponce de León et

Raga, 2006

[syns. Echinosoma peposacae Porta, 1914; E. peposacae (Porta, 1914) Travassos 1926; Corynosoma peposacae (Porta, 1914) Travassos, 1924]

GENUS Southwellina Witenberg, 1932

[syn. Hemiechinosoma Petrochenko et Smogorjevskaya, 1962]

\section{SPECIES}

S. hispida (Van Cleave, 1925) Witenberg, 1932 (type species) [syns. Arhythmorhynchus duocinctus Chandler, 1935; A. fuscus Harada, 1929; A. hispidus Van Cleave, 1925; A. quadrivirgata Yamaguti, 1935; Polymorphus ardeae Belopolskaya, 1958; Hemiechinosoma ardeae (Belopolskaya, 1958) Petrochenko et. Smogorjevskaya,1962; H. ponticum Petrochenko et Smogorjevskaya, 1962; Southwellina ardeae (Belopolskaya, 1958) Golvan, 1994]

S. macracanthus (Ward et Winter, 1952) Schmidt, 1973

[syn. Arhythmorhynchus macracanthus Ward et Winter, 1952]

S. sacra Bhattacharya, Pande et Srivastava, 2002,

CLASS POLYACANTHOCEPHALA Amin, 1987

(The validity of this class was supported by ribosomal RNA gene sequence studies by García-Varela et al. 2002.)

\section{ORDER POLYACANTHORHYNCHIDA Amin, 1987}

FAMILY Polyacanthorhynchidae Golvan, 1956

(previously in Rhadinorhynchidae)

GENUS Polyacanthorhynchus Travassos, 1920

(nec 1926)

SPECIES

P. caballeroi Diaz-Ungria et Rodrigo, 1960

P. kenyensis Schmidt et Canaris, 1967

P. macrorhynchus (Diesing, 1851) Travassos, 1920

(nec Baylis, 1927) (type species)

[syns. Echinorhynchus macrorhynchus Diesing, 1856; Polyacanthorhynchus arcuatum (Diesing, 1851) Travassos, 1920]

P. rhopalorhynchus (Diesing, 1851) Travassos, 1920

[syn. Echinorhynchus rhopalorhynchus Diesing, 1851]

\section{APPENDIX I}

Fossil acanthocephalan taxa reported from China.

FAMILY Zhijinitidae Qian, 1978 [Fossil]

GENUS Cambroclavus Mambetov in Mambetov et Repina, 1979

SPECIES

C. paradoxus Yi et Yin, 1984

GENUS Parazhijinites Yi et Yin, 1984

SPECIES

P. quizhouensis Yi et Yin, 1984

GENUS Zhijinites Yi, 1978

SPECIES

Z. cordiformis Yi et Yin, 1984

Z. panduriformis Yi et Yin, 1984

Z. triangularis Yi et Yin, 1984

\section{APPENDIX II}

Acanthocephalan genera incertae sedis assigned to Echinorhynchus sensu lato.

E. acanthotrias von Linstow, 1883

E. alcedinis Westrumb, 1882

E. amphipacus Westrumb, 1821

E. astacifluviatilis Diesing, 1851

E. bipennis Kaiser, 1893

E. blenni Rudolphi, 1810

E. corrugatus Sars, 1885

E. dendrocopi Westrumb, 1821

E. depressus Diesing, 1851

E. diffluens Zenker, 1832

E. eperlani von Linstow, 1884

E. galbulae Diesing, 1851

E. garzae Zeder, 1803

E. gazae Gmelin, 1790

E. hexacanthus Dujardin, 1845

E. hominis Leuckart, 1876

E. inflexus Cobbold, 1861

E. labri Rudolphi, 1819

E. lateralis Leidy, 1851

E. lendix (Phipps, 1774) de Marval, 1905

[syn. Sipunculus lendix Phipps, 1774]

E. magretti Parona, 1885

E. nardoi Molin, 1859

E. nitzschi Giebel, 1866

E. orestiae Neveu-Lamaire, 1905

E. pachyacanthus Sonsino, 1889

E. pardi Huxley in Ihering, 1902

E. pari Rudolphi, 1819

E. peleci Grimm, 1870

E. platessae Rudolphi, 1809

E. platessoides Gmelin, 1790

E. pleuronectis Gmelin, 1790

E. pleuronectisplatessoides Viborg, 1795

E. praetextus Molin, 1858

E. pupa von Linstow, 1905

E. putorii Molin, 1858

E. rhytidodes Monticelli, 1905

E. robustus Datta 1928

E. pseudosegmentatus Knupffer, 1888 
[syn. Moniliformis pseudosegmentatus Knupffer, 1888]

E. sciaenae Rudolphi, 1819

E. scopis Gmelin, 1790

E. scorpeanae Rudolphi, 1819

E. serpenulus Grimm, 1870

E. sipunculus Schrank, 1788

E. solitarium Molin, 1858

E. stridulae Goeze, 1782

E. striges Gmelin, 1782

E. taeniaeforme von Linstow, 1890
E. tardae Rudolphi, 1809

E. tenuicollis Froelich, 1802

E. urniger Dujardin, 1845

Acknowledgements. I would like to express my special gratitude and deep appreciation for Petra Rozkošná and Tomáš Scholz of Folia Parasitologica. The completion of this work would have not been possible without their meticulous attention to detail, dogged determination to achieve excellence, patience, conviction, and friendship.

\section{REFERENCES}

Acholonu A.D. 1969: Acanthocephala of Louisiana turtles with a redescription of Neoechinorhynchus stunkardi Cable et Fisher, 1961. Proc. Helminthol. Soc. Wash. 36: 177-183.

Alcántar-Escalera F.J., García-Varela M., VázquezDominguez E., Pérez-Ponce de León G. 2013: Using DNA barcoding to link cystacanths and adults of the acanthocephalan Polymorphus brevis in central Mexico. Mol. Ecol. Resources: in press.

Al Ghamdi A.O. 2013: Description of Rhadinorhynchus dorsoventrospinosus (Acanthocephala: Rhadinorhynchidae) from the red spot emperor Lethrinus lentjan with new host and locality records in Saudi Arabia. J. Egypt. Soc. Parasitol. 43: 209-214.

Amin O.M. 1982: Acanthocephala. In: S.P. Parker (Ed.), Synopsis and Classification of Living Organisms. McGraw-Hill Book Co., New York, pp. 933-941.

Amin O.M. 1985: Classification. In: D.W.T. Crompton and B.B. Nickol (Eds.), Biology of the Acanthocephala. Cambridge University Press, London and New York, pp. 27-72.

Amin O.M. 1987: Key to the families and subfamilies of Acanthocephala, with the erection of a new class (Polyacanthocephala) and a new order (Polyacanthorhynchida). J. Parasitol. 73: 1216-1219.

Amin O.M. 1992: Review of the genus Polymorphus Lühe, 1911 (Acanthocephala: Polymorphidae) with the synonymization of Hexaglandula Petrochenko, 1950, and Subcorynosoma Khokhlova, 1967, and a key to the species. Qatar Univ. Sci. J. 12: $115-123$.

Amin O.M. 2000: Acanthocephala in the Neotropical region. In: G. Salgado-Maldonado, A.N. García Aldrete and V.M. VidalMartínez (Eds.), Metazoan Parasites in the Neotropics: A Systematic and Ecological Approach. Inst. Biol., Univ. Nac. Aut. Mexico, Mexico City, Mexico, pp. 167-174.

Amin O.M. 2002: Revision of Neoechinorhynchus Stiles et Hassall, 1905 (Acanthocephala: Neoechinorhynchidae) with keys to 88 species in two subgenera. Syst. Parasitol. 53: 1-18.

Amin O.M. 2005: Occurrence of the subgenus Acanthosentis Verma et Datta, 1929 (Acanthocephala: Quadrigyridae) in Japan, with the description of Acanthogyrus (Acanthosentis) alternatspinus $\mathrm{n}$. sp. and $A$. (A.) parareceptaclis $\mathrm{n}$. sp. from Lake Biwa drainage fishes and a key to the species of the subgenus. Syst. Parasitol. 60: 125-137.

Amin O.M., Abdullah S.M.A., Mhaisen F.T. 2003: Description of Pomphorhynchus spindletruncatus n. sp. (Acanthocephala: Pomphorhynchidae) from freshwater fishes in northern Iraq, with the erection of a new pomphorhynchid genus, Pyriproboscis $\mathrm{n}$. gen., and keys to the genera of Pomphorhynchidae and the species of Pomphorhynchus Monticelli, 1905. Syst. Parasitol. 54: $229-235$.
Amin O.M., Canaris A.G., Kinsella J.M. 1999: A taxonomic reconsideration of the genus Plagiorhynchus s. lat. (Acanthocephala: Plagiorhynchidae), with descriptions of South African Plagiorhynchus (Prosthorhynchus) cylindraceus from shore birds and $P$. (P.) malayensis, and a key to the species of the subgenus Prosthorhynchus. J. Helminthol. Soc. Wash. 66: $123-132$.

Amin O.M., Evans P., Heckmann, R.A., El-Naggar A.M. 2013: The description of Mediorhynchus africanus n. sp. (Acanthocephala: Gigantorhynchidae) from galliform birds in Africa. Parasitol. Res. 112: 2897-2906.

Amin O.M., Dezfuli B.S. 1995: Taxonomic notes on Polyacanthorhynchus kenyensis (Acanthocephala: Polyacanthorhynchidae) from Lake Naivasha, Kenya. J. Parasitol. 81: 76-79.

Amin O.M., Ha N.V. 2008: On a new acanthocephalan family and a new order, from birds in Vietnam. J. Parasitol. 94: 1305-1310.

Amin O.M., Ha N.V., Heckmann R.A. 2008: New and already known acanthocephalans from amphibians and reptiles in Vietnam, with keys to species of Pseudoacanthocephalus Petrochenko, 1956 (Echinorhynchidae) and Sphaerechinorhynchus Johnston and Deland, 1929 (Plagiorhynchidae). J. Parasitol. 94: 181-189.

Amin O.M., Heckmann R.A., Ha N.V. 2011a: Description of Heterosentis holospinus $\mathrm{n}$. sp. (Acanthocephala: Arhythmacanthidae) from the striped eel catfish, Plotosus lineatus, in Halong Bay, Vietnam, with a key to species of Heterosentis and reconsideration of the subfamilies of Arhythmacanthidae. Comp. Parasitol. 78: 29-38.

Amin O.M., Heckmann R.A., Ha N.V. 2011b: Description of two new species of Rhadinorhynchus (Acanthocephala, Radinorhynchidae) from marine fish in Halong Bay, Vietnam, with a key to species. Acta Parasitol. 56: 67-77.

Amin O.M., Heckmann R.A., Ha N. V., Luc P.V., Doanh P.N: 2000: Revision of the genus Pallisentis (Acanthocephala: Quadrigyridae) with the erection of three new subgenera, the description of Pallisentis (Brevitritispinus) vietnamensis subgen. et sp. n., a key to species of Pallisentis, and the description of a new quadrigyrid genus, Pararaosentis gen. n. Comp. Parasitol. 67: 40-50.

Amin O.M., Heckmann R.A., Radwan N.A.E., Mantuano Anchundia J.S., Zambrano Alcivar M.A. 2009: Redescription of Rhadinorhynchus ornatus (Acanthocephala: Rhadinorhynchidae) from skipjack tuna, Katsuwonus pelamis, collected in the Pacific Ocean off South America, with special reference to new morphological features. J. Parasitol. 95: 656-664.

Amin O.M., Margolis L. 1998: Redescription of Bolbosoma capitatum (Acanthocephala: Polymorphidae) from false killer whale off Vancouver Island, with taxonomic reconsideration of 
the species and synonymy of $B$. physeteris. J. Helminthol. Soc. Wash. 65: 179-188.

Amin O.M., Reduin M. J. 1980: The effect of host species on growth and variability of Echinorhynchus salmonis Müller, 1784 (Acanthocephala: Echinorhynchidae), with special reference to the status of the genus. Syst. Parasitol. 2: 9-20.

Amin O.M., Sey O. 1996: Acanthocephala from Arabian Gulf fishes, with descriptions of Neoechinorhynchus dimorphospinus sp. n. (Neoechinorhynchidae), Tegorhynchus holospinus sp. n. (Illiosentidae), Micracanthorhynchina kuwaitensis sp. $\mathrm{n}$. (Rhadinorhynchidae), and Slendrorhynchus breviclaviproboscis gen. n., sp. n. (Diplosentidae), and a key to species of the genus Micracanthorhynchina. J. Helminthol. Soc. Wash. 63: 201-210.

Aznar F.J., Pérez-Ponce de León G., Raga J.A. 2006: Status of Corynosoma (Acanthocephala: Polymorphidae) based on Anatomical, ecological, and phylogenetic evidence, with the erection of Pseudocorynosoma, n. gen. J. Parasitol. 92: 548-564.

Bhattacharya S.B. 2007: Handbook on Indian Acanthocephala. Kolkata, Zool. Surv. India, 255 pp.

Bravo-Hollis M. 1946: Neoechinorhynchus emydis (Leidy, 1852); Van Cleave, 1913, parásite del intestine de Chrysemys ornate. Ann. Inst. Biol. Univ. Nac. Aut. México 17: 187-192.

Bray R.A., Spencer Jones M.E., Lewis J.W. 1988: Acanthocephaloides cyrusi n. sp. (Acanthocephala: Arhythmacanthidae) from southeast African teleostean fishes. Syst. Parasitol. 12: 109-116.

Cable R.M., Linderoth J. 1963: Taxonomy of some Acanthocephala from marine fishes with reference to species from Curaçao, N.A., and Jamaica, W.I. J. Parasitol. 49: 706-716.

ChandLer A.C. 1934: A revision of the genus Rhadinorhynchus (Acanthocephala) with descriptions of new genera and species. Parasitology 26: 352-358.

Chander A.C. 1941: The specific status of Moniliformis (Acanthocephala) of Texas rats, and a review of the species of this genus in the western hemisphere. J. Parasitol. 27: 241-244.

Chandra K.J., Rekha Z., Rao K.H. Shyamasundari K. 1982: Neoechinorhynchus chilkaensis Podder, 1937. Indian J. Parasitol. 6: 293-294.

Chen C.L. 1973: An illustrated guide to the fish diseases and causative pathogenic fauna and flora in the Hubie Province. Sci. Press (Inst. Hydrobiol., Acad. Sinica), Bejing, 271 pp.

Chervy L. 2009: Unified terminology for cestode microtriches: a proposal from the International Workshops on Cestode Systematics in 2002-2008. Folia Parasitol. 56: 199-230.

Cholodkovsky N.A. 1897: [Classification of the Acanthocephala]. Trudy Sankt Petersburg. Obshchestva Estestvoispytatelei 28: 14-20. (In Russian).

Снuвв J.C. 2004: Echinorhynchus salmonis Müller, 1784 absent in Britain and Ireland: re-identification of museum specimens. Folia Parasitol. 51: 72-74.

DatTa M.N. 1936: Scientific results of the Yale North India Expedition. XX. Helminth parasites of fishes from North India with special reference to Acanthocephala. Biol. Rep. XX. Res. Ind. Mus. 38: 211-229.

Dezfuli B.S., Tinti F. 1998: Species recognition of congeneric acanthocephalans in slider turtles by random amplified polymorphic DNA (RAPD) markers. J. Parasitol. 84: 860-862.

Dhar R.L., Kharoo V.K. 1984: A new species of Acanthocephala, Neoechinorhynchus glyptosternumi $\mathrm{n}$. sp. from the intestine of a Kashmir fish, Glyptosternum sp. Ind. J. Helminthol. 36: 36-39.

Dogiel V.A., Bychowsky B.E. 1938: Fish parasites of the Caspian Sea. Kom. Izuch. Kasp. Morya 7: 1-149.
Dollfus R.PH. 1938: Etude morphologique et systématique de deux espèces d'acanthocéphales, parasites de Lémuriens et de Singes. Rev. Parasitol. Hum. Comp. 16: 385-422.

DolLfus R.PH. 1951: Miscellanea Helminthologica Maroccana. IIII. Quelques Trématodes, Cestodes et Acanthocéphales. Arch. Inst. Pasteur Maroc 4: 104-229.

Dollfus R.PH. 1953: Miscellanea helminthological Maroccana. XI. Sur cinq espèces d'acanthocéphales don't une du hérisson Aethechinus algirus (Duvernoy et Lereboullet, 1840). Arch. Inst. Pastuer Maroc 4: 541-562.

DUDIŇÁK V., ŠNÁBEL V. 2001: Comparative analysis of Slovak and Czech populations of Pomphorhynchus laevis (Acanthocephala) using morphological and isoenzyme analysis. Acta Zool. Univ. Comen. 44: 41-50.

Edmonds S.J. 1957: Acanthocephala. Report of the British, Australian, New Zealand Antarctic Research Expedition, 19291931. The Adelaide Research Committee, 1937-1938, Ser. B, Vol. 6: 93-98.

EDmonds S.J. 1982: Australian Acanthocephala. No. 15: Four species. Trans. Roy. Soc. S. Austr. 106: 71-76.

FAROoQI H.U. 1989: Redescription of Acanthogyrus acanthogyrus (Thapar, 1927) (Acanthocephala: Acanthogyridae) and its taxonomic status. Riv. Parassitol. 4: 157-163.

Florescu B.I., Ienistea M.A. 1984: Aperçu sur les acanthocéphales de Roumanie (Acanthocephala). Travaux Mus. Hist. Natur. Grigore Antipa 25: 7-45.

Fontaneto D., Jondelius U. 2011: Broad taxonomic sampling of mitochondrial cytochrome $\mathrm{c}$ oxidase subunit 1 does not solve the relationship between Rotifera and Acanthocephala. Zool. Anz. 250: 80-85.

FukUi T. 1929: On some Acanthocephala found in Japan. Annot. Zool. Jap. 12: 225-270.

Fukui T., Morisita T. 1937: Studies on the Acanthocephala of Japan. Zikken. Igaku. Zasshi. 21: 36-41 (in Japanese.)

García-Varela M., Cummings M.P., Pérez-Ponce de León G., Gardner S.L., Laclette J.P. 2002: Phylogenetic analysis based on $18 \mathrm{~S}$ ribosomal RNA gene sequences supports the existence of class Polyacanthocephala (Acanthocephala). Mol. Phylogen. Evol. 23: 288-292.

García-Varela M., GonzÁlez-Oliver A. 2008: The systematic position of Leptorhynchoides (Kostylew, 1924) and Pseudoleptorhynchoides (Salgado-Maldonado, 1976), inferred from nuclear and mitochondrial DNA gene sequences. J. Parasitol. 94: 959-962.

García-Varela M., Nadler S. A. 2005: Phylogenetic relationships of Alaeacanthocephala (Acanthocephala) inferred from SSU and LSU rRNA gene sequences. J. Parasitol. 91: 14011409.

García-Varela M., Nadler S.A. 2006: Phylogenetic relationships among Syndermata inferred from nuclear and mitochondrial gene sequences. Mol. Phylog. Evol. 40: 61-72.

García-Varela M., Pérez-Ponce de León G. 2008: Validating the systematic position of Profilicollis Meyer, 1931 and Hexaglandula Petrochenko, 1950 (Acanthocephala: Polymorphidae) using cytochrome C oxidase (COX 1). J. Parasitol. 94: 212-217.

García-Varela M., Pérez-Ponce de León G., Aznar F.J., NaDLER S.A. 2009: Systematic position of Pseudocorynosoma and Andracantha (Acanthocephala, Polymorphidae) based on nuclear and mitochondrial gene sequences. J. Parasitol. 95: $178-185$.

García-Valera M., Pérez-Ponce de León G., Aznar F.J., NADLER S.A. 2011: Erection of Ibirhynchus gen. nov. (Acanthocephala: Polymorphidae), based on molecular and morphological data. J. Parasitol. 97: 97-105. 
García-Varela M., Pérez-Ponce de León G., de La Torre P., Cummings M.P., Sarma S.S.S., Laclette J.P. 2000: Phylogenetic relationships of Acanthocephala based on analysis of 18S ribosomal RNA gene sequences. J. Mol. Evol. 50: 532-540.

Garey J.R., Near T.J., Nonnemacher M.R., Nadler S.A. 1996: Molecular evidence for Acanthocephala as a subtaxon of Rotifera. J. Mol. Evol. 43: 287-292.

Garey J.R., Schmidt-Rhaesa A., Near T.J., Nadler S.A. 1998: The evolutionary relationships of rotifers and acanthocephalans. Hydrobiologia 387: 83-91.

Golvan Y.J. 1956: Acanthocéphales d'oiseaux. Première note. Description d'Arhythmorhynchus longicollis (Villot, 1875) et revision du genre Arhythmorhynchus Lühe, 1911 (Acanthocephala). Ann. Parasitol. Hum. Comp. 31: 199-224.

Golvan Y.J. 1959: Le Phylum des Acanthocephala. Deuxième note. La Classe de Eoacanthocephala (Van Cleave, 1936). Ann. Parasitol. Hum. Comp. 34: 5-52.

Golvan Y.J. 1960-1961: Le Phylum des Acanthocephala. Troisième note. La Classe des Palaeacanthocephala (Meyer, 1931). Ann. Parasitol. Hum. Comp. 35: 76-91, 138-165, 350-386, 573-593, 713-723; 36: 76-91, 612-647, 717-738.

Golvan Y.J. 1965: Acanthocéphales de Madagascar récoltés par E. R. Brygoo. Ann. Parasitol. Hum. Comp. 40: 303-316.

Golvan Y.J. 1969: Systematique des Acanthocephales (Acanthocephala Rudolphi, 1801), L'ordre des Palaeacanthocephala Meyer, 1931, La superfamille des Echinorhynchidea (Cobbold, 1876) Golvan et Houin 1963. Mém. Mus. Nat. Hist. 47: 1-373.

Golvan Y.J. 1994: Nomenclature of the Acanthocephala. Res. Rv. in Parasitol. 54: 135-205.

Gupta N.K., LAtA V. 1967: Observations on eight already known acanthocephalan parasites from vertebrate hosts. Res. Bull. Panjab Univ. Sci. 18: 325-341.

Haley A.J., Bullock W.L. 1953: A new species of Acanthocephala from the sunfish Lepomis gibbosus (Linnaeus) with a redescription of the family Fessisentidae Van Cleave, 1931. Am. Midl. Nat. 50: 202-205.

Hamann O. 1892: Das system der Acanthocephalen. Zoologischer Anzieger 15: 195-197.

Harada I. 1935: Zur Acanthocephalenfauna von Japan. Mem. Fac. Sci. Agr., Taihoku Imp. Univ. Formosa, Japan 14: 7-23.

Harrtington R.W., Pillbury N.R. Jr. 1938: Preliminary report on the internal parasites of the herring gull. Bull. Bowdoin Coll. 4: 8-9.

Hartwich G. 1956: Südamerikanische Acanthocephalen aus der Zoologischen Sammlung des Bayerischen Staats. Zool. Anz. 156: 299-308.

Hassanine R.M.EL-S. 2006: Acanthocephalans from Red Sea fishes. Family Cavisomidae Meyer, 1932: the seasonal cycle of Diplosentis nudus (Harada, 1938) Pichelin et Cribb, 2001 in a definitive fish host, and a comment on Sclerocollum Schmidt et Paperna, 1978. Acta Parasitol. 51: 123-129.

Herlyn H. 2001: First description of an apical epidermis cone in Paratenuisentis ambiguous (Acanthocephala: Eoacanthocephala) and its phylogenetic implications. Parasitol. Res. 87: 306-310.

Jellison W.L., Neiland K.A. 1965: Parasites of Alaskan vertebrates. Host-parasite index. Univ. Oklahoma, Res. Inst., Norman, Oklahoma Project 1508. 73 pp.

Johnston T.H., Best E.W. 1951: Acanthocephala. Australasian Antarctic Expedition 1911-14. Sci. Rep. Ser. C. Zool. Bot. 10: $5-20$

Johnston T.H., Edmonds S.J. 1947: Australian Acanthocephala. 6. Rec. S. Austr. Mus. 8: 555-562.
Johnston T.H., Edmonds S.J. 1951: Australian Acanthocephala. 8. Trans. Roy. Soc. S. Austr. 74: 1-5.

Khatoon N., Bilqees F.M. 1991: Classification of the Acanthocephala - a review. Proc. Parasitol. 11: 22-70.

Khatoon N., Bilqees F.M. 2011: Acanthocephala of vertebrates. A world record. Saarbrücken, Germany: VDM Verlag, Dr. Müller GmbH and Co., 566 pp.

Khoкhlova I.G. 1975: [Revision of Arhythmorhynchus Lühe, 1911 (Acanthocephala: Polymorphidae)]. Trudy GELAN 25: 195-203. (In Russian).

Khoкhlova I.G. 1986: [The acanthocephalan fauna of terrestrial vertebrates of SSSR]. Nauka, Moskva. 276 pp. (In Russian).

Kostylew N.N. 1922: Sur acanthocéphales de l'eider (Somateria mollissima). Parasitology 14: 372-377.

Kristensen R.M. 2002: An introduction to Loricifera, Cycliophora, and Micrognathozoa. Integr. Comp. Biol. 42: 641-652.

Leiper R.T., Atkinson E.L. 1915: Parasitic worms, with a note on a free-living nematode. Nat. Hist. Rep. Brit. Antar. (Terra Nova) Exp. 1910. Nat. Hist. Rep., Zool. 3: 19-60.

Leuckart R. 1848: Über die Morphologie und Verwandtschaftsverhältnisse der wirbellosen Thiere. Ein Beitrag zur Charakteristik und Classification der Thierischen Formen. F. Vieweg und Sohn, Braunschweig, 180 pp.

Linton E. 1891: Notes on Entozoa of marine fishes, with descriptions of new species. Part III. Acanthocephala. Rep. U.S. Comm. Fish Fisher. 1888. 16: 523-542.

Linton E. 1892: Notes on avian Entozoa. Proc. U. S. Nat. Mus. 15: $87-113$.

LÜHE M. 1904-1905: Geschichte und Ergebnisse der Echinorhynchen-Forschung bis auf Westrumb (1821) (Mit Bemerkungen über alte und nueu Gattungen der Acanthocephalen). Zool. Annal. Zeit. Gesch. Zool., Würzburg 1: 139-353.

LÜHE M. 1911: Acanthocephalen. Register der Acanthocephalen und parasitischen Plattwürmer geordnet nach ihern Wirten. Prof. Dr. Brauer, Die Süsswasserfauna Deutschlands, Eine Exkursionsfauna, Jena 16: 114 pp.

Lyster L.L. 1940: Parasites of some Canadian sea mammals. Can. J. Res. 18: 395-409.

Machado Filho D.A. 1946: Sobre Moniliformis moniliformis (Bremser), Moniliformis travassosi Meyer, 1932, e outra espécies duvidosas do gênero (Acanthocephala). Bol. Esc. Nac. 1: $13-32$.

Machalska J. 1981: Helminth fauna of birds of the genus Turdus examined during their spring and autumn migration. II. Acanthocephalans. Acta Parasitol. Pol. 28: 171-177.

Margolis, L. 1955: Corynosoma hadweni Van Cleave, a probable synonym of $C$. wegeneri Heinze (Acanthocephala). J. Parasitol. 41: 326-327.

Martinez-Aquino A., Reyna-Fabián M.E., Rosas-Valdez R., Razo-Mendivil U., de León G.P., García-Valera M. 2009: Detecting a complex of cryptic species within Neoechinorhynchus golvani (Acanthocephala: Neoechinorhynchidae) inferred from ITSs and LSU rDNA gene sequences. J. Parasitol. 95: 1040-1047.

DE Marval L. 1905: Monographie des Acanthocéphales d'oiseaux. Rev. Suisse Zool. 13: 95-387.

Meyer A. 1931: Neue Acanthocephalen aus dem Berliner Museum. Burgründung eines neue Acanthocephalen Systems auf Grund einer Untersuchung der Berliner Sammlung. Zool. Jahr.. Abt. System., Ökol. Geograph. Tiere 62: 53-108.

Meyer A. 1932: Acanthocephala. Dr. H.G. Bronn's Klassen und Ordnungen des TierReichs. Akad. Verlag, Leipzig, 4: 1-332. 
Meyer A. 1933: Acanthocephala. In: Dr. H.G. Bronn's Klassen und Ordnungen des TierReichs. Leipzig: Akad. Verlag. MBH 4: $333-582$.

Miкhailova E.I. 2013: Origination of a separate form of Neoechinorhynchus salmonis Ching, 1984 (Acanthocephales: Neoechinorhynchidae) in severe environment of the Asian Arctic. Parasitol. Res. 112: 1973-1981.

Min G.S., PARK J.K. 2009: Eurotatorian paraphyly: revisiting phylogenetic relationships based on the complete mitochondrial genome sequence of Rotaria rotatoria (Bdelloidea: Rotifera: Syndermata). BMC Genomics 10: 533.

Monks S. 2001: Phylogeny of the Acanthocephala based on morphological characters. Syst. Parasitol. 48: 81-116.

Monks S., Pulido-Flores G., Violante-González J. 2011: A new species of Neoechinorhynchus (Acanthocephala: Neoechinorhynchidae) in Dormitator latifrons (Perciformes: Eleotridae) from the Pacific coast of Mexico. Comp. Parasitol. 78: 21-28.

Monks S., Richardson D.J. 2011: Phylum Acanthocephala Kohlreuther, 1771. In: Z.-Q. Zhang (Ed.), Animal Biodiversity: An Outline of Higher-Level Classification and Survey of Taxonomic Richness. Zootaxa 3148: 234-237.

Monticelli F.S. 1887: Osservazioni intorno ad alcune specie di Acantocefali. Bull. Soc. Natur. Napol., 1: 1-11, 19-29.

Nama H.S., Rathore G.S. 1984: The acanthocephalan genus Centrorhynchus occurring in Indian birds with description of two new species from Rajasthan. Ind. J. Helminthol. 34: 143-150.

NeAR T.J. 2002: Acanthocephalan phylogeny and the evolution of parasitism. Integ. Comp. Biol. 42: 668-677.

Near T.J., Garey J.R., Nadler S.A. 1998: Phylogenetic relationships of the Acanthocephala inferred from 18S ribosomal DNA sequences. Mol. Phylogen. Evol. 10: 287-298.

Nickol B.B., Crompton D.W.T., Searle D.W. 1999: Reintroduction of Profilicollis Meyer, 1931, as a genus in Acanthocephala: significance of the intermediate host. J. Parasitol. 85: 716-718.

Nickol B.B., Heard R.W., Smith N.F. 2002: Acanthocephalans from crabs in the southeastern U.S., with the first intermediate hosts known for Arhythmorhynchus frassoni and Hexaglandula corynosoma. J. Parasitol. 88: 79-83.

O’Mahony E.M., Kennedy C.R., Holland C.V. 2004: Comparison of morphological characters in Irish and English populations of the acanthocephalan Pomphorhynchus laevis (Müller, 1776). Syst. Parasitol. 59: 147-157.

ORTLEPP R.J. 1924: On a collection of helminths from Dutch Guiana. J. Helminthol. 2: 15-40.

Perrot-Minnot M.J. 2004: Larval morphology, genetic divergence, and contrasting levels of host manipulation between forms of Pomphorhynchus laevis (Acanthocephala). Int. J. Parasitol. 34: 45-54.

Petrochenko V.I. 1952: [On the position of the Acanthocephala in the zoological system. (Phylogenetic connections of the Acanthocephala with other groups of invertebrates)] Zool. Zhr. 31: 288-327. (In Russian.)

Petrochenko V.I. 1953: Thorny-headed worms (Acanthocephala) of U.S.S.R. amphibians: A.M. Petrov (Ed.) In: Contributions to Helminthology publications to Commemorate the 75 birthday of K.I Skrjabin. Acad. Nauk S.S.S.R., Moscow, Russia, 508-517.

Petrochenko V.I. 1956: [Acanthocephala of Domestic and Wild Animals]. Vol. 1. Moscow: Izdatel'stvo Akad. Nauk SSSR. (In Russian.) (English translation by Israel Program for Scientific Translations, Ltd., Jerusalem, 1971, 465 pp.)
Petrochenko V.I. 1958: [Acanthocephala of Domestic and Wild Animals]. Vol. 2. Moscow: Izdatel'stvo Akad. Nauk SSSR. (In Russian.) (English translation by Israel Program for Scientific Translations, Ltd., Jerusalem, 1971, 478 pp.)

Pichelin S., Cribi T.H. 1999: A review of the Arhythmacanthidae (Acanthocephala) with a description of Heterosentis hirsutus n. sp. from Cnidoglanis macrocephala (Plotosidae) in Australia. Parasite 6: 293-302.

Pichelin S., CRibB T.H. 2001: The status of Diplosentidae (Acanthocephala: Palaeacanthocephala) and a new family of acanthocephalans from Australian wrasses (Pisces: Labridae). Folia Parasitol. 48: 289-303.

Pinacho-Pinacho C.D., Pérez-Ponce de León G., GarcíaVarela M. 2012: Description of a new species of Neoechinorhynchus (Acanthocephala: Neoechinorhynchidae) a parasite of Dormitator latifrons from southwestern Mexico based on morphological and molecular characters. Parasitol. Int. 61: 634-644.

Porta A. 1908: Contributo allo studio degli acantocefali dei pesci. Biologia 1: 377-423.

Porta A. 1909: Gli acantocefali dei mammiferi. Arch. Zool., Napoli 4: 239-285.

RADWAN N.A. 2012: Phylogenetic analysis of Sphaerirostris picae (Acanthocephala: Centrorhynchidae) based on large and small subunit ribosomal DNA gene. Int. J. Parasitol. Res. 4: 106-110.

Redi F. 1684: Osservazioni Interna Agli Animali Viventi che si Trovano Regli Animali Viventi. Firenze, 253 pp.

Rieger R.M., Tyler S. 1995: Sister-group relationship of Gnathostomulida and Rotifera - Acanthocephala. Invert. Biol. 114: 186-188.

Rosas-Valdez R., Morrone J.J., García-Varela M. 2012: Molecular phylogenetics of Floridosentis Ward, 1953 (Acanthocephala: Neoechinorhynchidae) parasites of mullets (Osteichthyes) from Mexico, using 28S rDNA sequences. J. Parasitol. 98: 855-862.

Rudolphi C.A. 1802: Fortsetzung der Beobachtungen uber die Eingeweidwurmer. Arch. Zool. Zootom. 2: 1-67.

Salgado-Maldonado G. 2006: Checklist of helminth parasites of freshwater fishes from Mexico. Zootaxa 1324: 1-357.

Salgado-Maldonado G., Amin O.M. 2009: Acanthocephala of the Gulf of Mexico. In: D.L. Felder and D.K. Camp (Eds.), Gulf of Mexico. Origin, Waters, and Biota. Vol. 1, Biodiversity. Texas University Press, Corpus Christi, Texas, pp. 539-552.

Schmidt G.D., Dailey M.D. 1971: A zoogeographic note on the acanthocephalan Corynosoma bullosum (von Linstow, 1892). Trans. Amer. Microsc. Soc. 90: 94-95.

Schmidt G.D., Kuntz R.E. 1966: New and little known plagiorhynchid Acanthocephala from Taiwan and the Pescadores Islands. J. Parasitol. 52: 520-527.

Schmidt G.D., Kuntz R.E. 1977: Revision of Mediorhynchus Van Cleave, 1916 (Acanthocephala) with a key to species. J. Parasitol. 63: 500-507.

Shin H.-H., Chen H.-Y., LeE C.-Y. 2010: Acanthocephalan fauna of marine fish in Taiwan and the differentiation of three species by ribosomal DNA sequences. Taiwania 55: 123-127.

SkRJABIN K.I., Shults R.E.S. 1931: [Helminthoses of Man (Foundations of Medical Helminthology) for Medical Doctors and Veterinarians, Biologists and Students.] Part II. State Medical Publisher, Moscow and Leningrad, 250 pp. (In Russian.)

Smales L.R. 2012: A new acanthocephalan family, the Isthmosacanthidae (Acanthocephala: Echinorhynchida), with the description of Isthmosacanthus fitzoyensis n. gen., n. sp. from 
threadfin fishes (Polynemidae) of northern Australia. Syst. Parasitol. 82: 105-111.

Sobecka E., Szostakowska B., Mackenzie K., Hemmingsen W., Prajsnar S., Eydal M. 2012: Genetic and morphological variation in Echinorhynchus gadi Zoega in Müller, 1776 (Acanthocephala: Echinorhynchidae) from Atlantic cod Gadus morhua L. J. Helminthol. 86: 16-25.

Southwell T., Macfie J.W.S. 1925: On a collection of Acanthocephala in the Liverpool School of Tropical Medicine. Ann. Trop. Med. Parasitol. 19: 141-184.

Špakulová M., Perrot-Minnot M., Neuhaus B. 2011: Resurrection of Pomphorhynchus tereticollis (Rudolphi, 1809) (Acanthocephala: Pomphorhynchidae) based on new morphological and molecular data. Helminthologia 48: 268-277.

Steinauer M.L., Nickol B.B. 2007: Cryptic speciation and patterns of phenotypic variation of a highly variable acanthocephalan parasite. Mol. Ecol. 16: 4097-4109.

Steinauer M.L., Nickol B.B., Broughton R., Orti G. 2005: First sequenced mitochondrial genome from the phylum Acanthocephala (Leptorhynchoides thecatus) and its phylogenetic position within Metazoa. J. Mol. Evolt. 60: 706-715.

Tantaleán M., Sánchez L., Gómez L., Huiza A. 2005: Acantocéfalos del Perú. Rev. Peruana Biol. 12: 1-19.

Thapar G.S. 1927: On Acanthogyrus n. gen. from the intestine of the Indian fish Labeo rohita, with a note on the classification of the Acanthocephala. J. Helminthol. 5: 109-120.

Tkach V.V., Lisitsyna O.I., Crossley J.L., Binh T.T., Bush S.F. 2013: Morphological and molecular differentiation of two new species of Pseudoacanthocephalus Petrochenko, 1958 (Acanthocephala: Echinorhynchidae) from amphibians and reptiles in the Philippines, with identification key for the genus. Syst. Parasitol. 85: 11-26.

Travassos L. 1925: Quelques acanthocèphales nouveaux. Comp. Ren. Hebdom. Séan. Soc. Biol. Paris 95: 935-937.

Travassos L. 1926: Contribuições para o conhecimento da fauna helminthologica brasileira. XX. Revisaeo dos acanthocefalos brasileiros. Part II. Fam. Echinorhynchidae. Sub-fam. Centrorhynchinae Travassos, 1919. Mem. Inst. Oswaldo Cruz 19: 31-125.

Väinölä R., Valtonen E.T., Gibson D.I. 1994: Molecular systematics in the acanthocephalan genus Echinorhynchus (sensu lato) in northern Europe. Parasitology 108: 105-114.

Van Cleave H.J. 1936: The recognition of a new order in the Acanthocephala. J. Parasitol. 22: 202-206.

Van Cleave H.J. 1941: Relationships of the Acanthocephala. Am. Natur. 75: 31-47.

Van Cleave H.J. 1945: The acanthocephalan genus Corynosoma. 1. The species found in water birds in North America. J. Parasitol. 31: 332-340.

Van Cleave, H.J. 1947: The Eoacanthocephala of North America, including the description of Eocollis arcanus, new genus and new species, superficially resembling the genus Pomphorhynchus. J. Parasitol. 33: 285-296.

Van Cleave H.J. 1948: Expanding horizons in the recognition of a phylum. J. Parasitol. 34: 1-20.

Van Cleave H.J. 1949: Morphological and phylogenetic interpretation of cement glands in the Acanthocephala. J. Morphol. 84: 427-457.

Van Cleave, H.J. 1951: Speciation and formation of genera in the Acanthocephala. Anat. Rec. 111: 525-526.
Van Cleave H.J. 1952: Acanthocephalan nomenclature introduced by Lauro Travassos. Proc. Helminthol. Soc. Wash. 19: $1-8$.

Van Cleave H.J. 1953: Acanthocephala of North American mammals. Ill. Biol. Monogr., 23: 1-179.

Van Cleave H.J., Rausch R L. 1951: The Acanthocephala of eider ducks. Proc. Helminthol. Soc. Wash. 18: 81-84.

Van Cleave H.J., Williams R.B. 1951: Acanthocephala from passerine birds in Alaska. J. Parasitol. 37: 151-159.

Verweyen L., Klimpel S., Palm H.W. 2011: Molecular phylogeny of the Acanthocephala (class Palaeacanthocephala) with a paraphyletic assemblage of the orders Polymorphida and Echinorhynchida. PLoS ONE 6: e28285.

WANG P.Q. 1986: Notes on Acanthocephala from Fujian, with descriptions of three new species. Wuyi Sc. J. 6: 181-192.

Wayland M.T., Gibson D.I., Sommerville C. 2005: Morphometic discrimination of two allozymically diagnosed sibling species of the Echinorhynchus gadi Zoega in Müller complex (Acanthocephala) in the North Sea. Syst. Parasitol. 60: 139-149.

Weber M., Wey-Fabrizius A.R., Podsiadlowski L., Witek A., Schill R.O., Sugar L., Herlyn H., Hankeln T. 2013: Phylogenetic analyses of endoparasitic Acanthocephala based on mitochondrial genomes suggest secondary loss of sensory organs. Mol. Phylogenet. Evol. 66: 182-189.

Welch D.B.M. 2000: Evidence from a protein-coding gene that acanthocephalans are rotifers. Invert. Biol. 119: 17-26.

Witek A., Herlyn H., Ebersberger I., Mark Welch D.B., HanKELN T. 2009: Support for the monophyletic origin of Gnathifera from phylogenomics. Mol. Phylog. Evol. 53: 1037-1041.

Witek A., Herlyn H., Meyer A., Boell L., Bucher G., HanKELN T. 2008: EST based phylogenomics of Syndermata questions monophyly of Eurotatoria. BMC Evol. Biol. 8: 345.

Witenberg G. 1932a: Akanthocephalen Studien. I. Uber einige für Systematik der Akanthocephalen wichtige anatomisch Merkmale. Boll. Zool., Publ. dall'Unione Zool. Ital. 3: 243-252.

Witenberg G. 1932b: Akanthocephalen Studien. II. Über das System der Acanthocephalen. Boll. Zool., Publ. Unione Zool. Ital. 3: $253-256$.

Yamaguti S. 1939: Studies on the helminth fauna of Japan. Part 29. Acanthocephala II. Jpn. J. Zool. 13: 317-351.

Yamaguti S. 1963: Acanthocephala. Systema Helminthum. Wiley Intersci., New York, London, 5: 1-423.

Zafar M.M., Faroodi H.U. 1981: Redescription of Plagiorhynchus nicobarensis (Soota and Kansal, 1972) n. comb., with comments on Centrorhynchus spilornae Schmidt et Kuntz, 1969. Ind. J. Parasitol. 5: 179-182.

Zdzitowiecki K. 1986: A contribution to the knowledge of morphology of Corynosoma bullosum (von Linstow, 1892) (Acanthocephala). Acta Parasitol. Pol. 30: 225-232.

Zdzitowiecki K. 1989: New data on the morphology and distribution of two acanthocephalans, Andracantha baylisi (Zdzitowiecki, 1986) comb. n. and Corynosoma australe Johnston, 1937. Acta Parasitol. Pol. 34: 167-172.

Zнuкоv E.V. 1960: Helminth endoparasites of marine fish in the Sea of Japan and south Kuril shallow waters. Trudy Zool. Inst., Akad. Nauk S.S.S.R. 28: 1-146.

ZRZAVÝ. J. 2001: The interrelationships of metazoan parasites: a review of phylum- and higher- level hypotheses from recent morphological and molecular phylogenetic analyses. Folia Parasitol. 48: 81-103. 


\section{INDEX OF ACANTHOCEPHALAN FAMILIES}

Apororhynchidae

Arhythmacanthidae

283

Cavisomidae

284

Centrorhynchidae

292

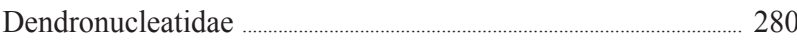

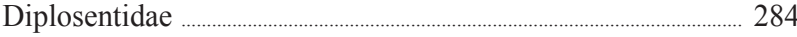

Echinorhynchidae ...................................................................................... 284

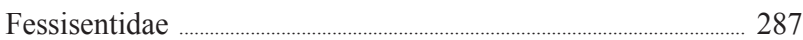

Gigantorhynchidae ...................................................................................... 288

Heteracanthocephalidae ............................................................................. 287

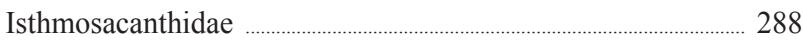

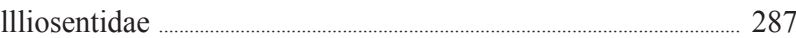

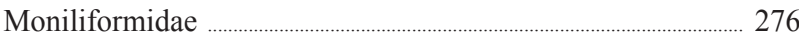

\section{INDEX OF ACANTHOCEPHALAN GENERA}

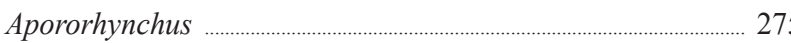

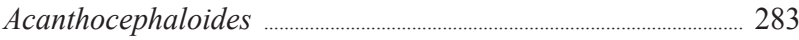

Acanthocephalus ................................................................................................ 285

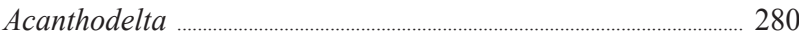

Acanthogyrus ............................................................................................... 278

Allorhadinorhynchus ............................................................................... 284

Amapacanthus ................................................................................................... 284

Andracantha ……................................................................................... 295

Anuracanthorhynchus .................................................................................. 285

Ardeirhynchus .......................................................................................... 295

Arhythmorhynchus .................................................................................... 295

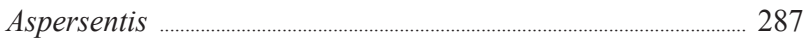

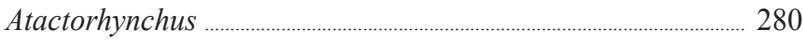

Australiformis ......................................................................................... 276

Australorhynchus ....................................................................................... 289

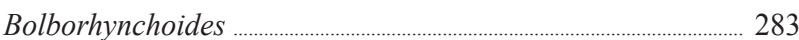

Bolbosoma ……………………………………………………………….... 296

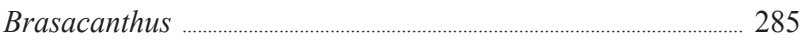

Breizacanthus ................................................................................................... 283

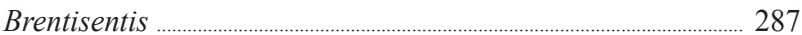

Bullockrhynchus ................................................................................... 287

Caballerorhynchus ................................................................................... 284

Cambroclavus ................................................................................................ 298

Cathayacanthus ......................................................................................... 290

Cavisoma ……………………………………………………………………..... 284

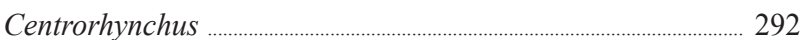

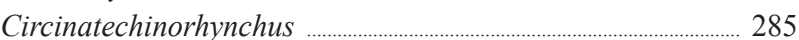

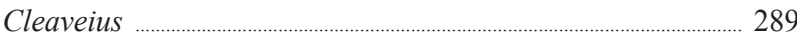

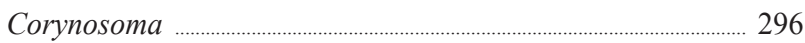

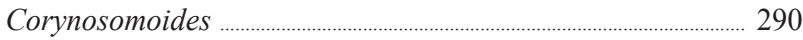

Cucullanorhynchus ...................................................................................... 277

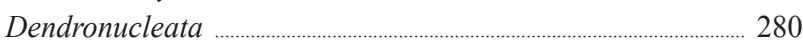

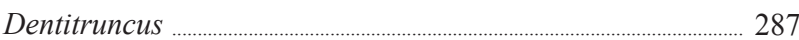

Diplosentis ..................................................................................................... 284

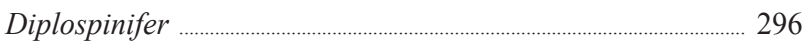

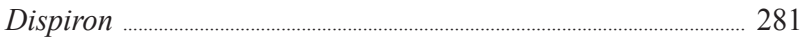

Dollfusentis ............................................................................................. 287

Neoechinorhynchidae ................................................................................. 280

Oligacanthorhynchidae ……………………………………………….... 277

Plagiorhynchidae .......................................................................................... 293

Polyacanthorhynchidae ......................................................................... 298

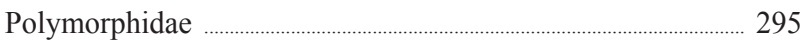

Pomphorhynchidae .................................................................................. 288

Pyrirhynchidae _............................................................................................... 291

Quadrigyridae ................................................................................................ 278

Rhadinorhynchidae ....................................................................................... 289

Sauracanthorhynchidae ........................................................................... 291

Tenuisentidae _........................................................................................................ 282

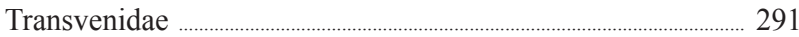

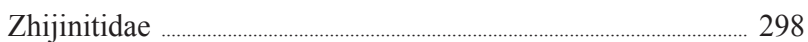

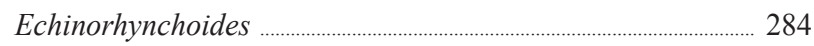

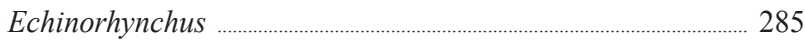

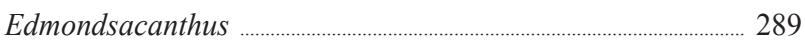

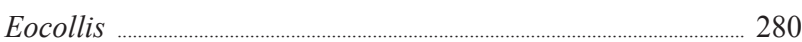

Euzetacanthus ................................................................................................ 283

Femogibbosus ................................................................................................ 284

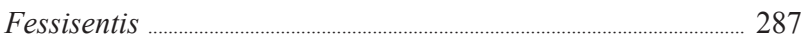

Filicollis ...................................................................................................... 297

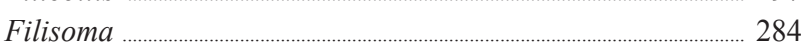

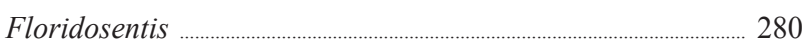

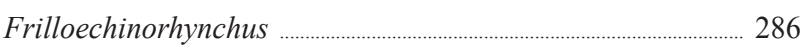

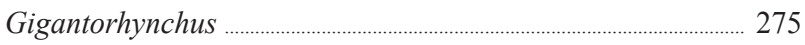

Goacanthus ............................................................................................... 287

Golvanacanthus ........................................................................................... 289

Golvanorhynchus ................................................................................. 284

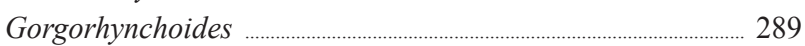

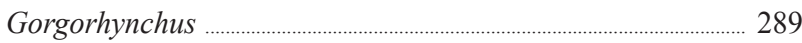

Gorytocephalus ............................................................................................. 281

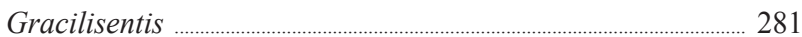

Heptamegacanthus .................................................................................. 277

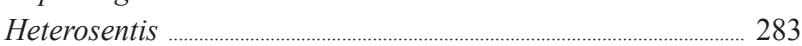

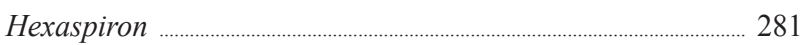

Hypoechinorhynchus ................................................................................ 283

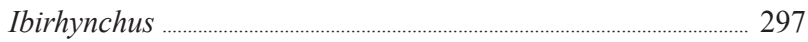

Indorhynchus .............................................................................................. 287

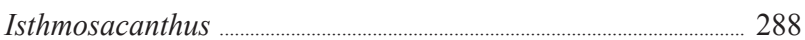

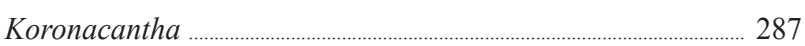

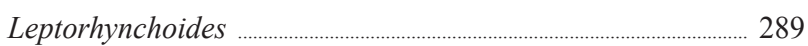

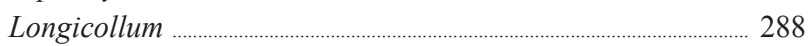

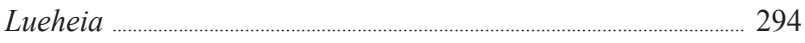

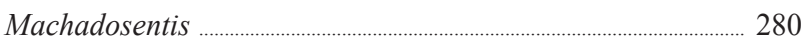

Macracanthorhynchus ......................................................................... 277

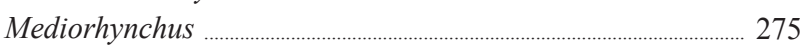

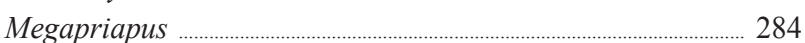

Megistacantha ............................................................................................ 290

Metacanthocephaloides ......................................................................... 290

Metacanthocephalus .................................................................................... 290 
Metarhadinorhynchus

Micracanthorhynchina

Microsentis

Moniliformis

Multisentis

Neoechinorhynchus

Neogorgorhynchoides

Neolacunisoma

Neoncicola

Neorhadinorhynchus

Nephridiacanthus

Octospinifer

Octospiniferoides

Oligacanthorhynchus

Oligoterorhynchus

Oncicola

Owilfordia

Pachysentis

Palliolisentis

Pallisentis

Pandosentis

Paracanthocephaloides

Paracanthorhynchus

Paracavisoma

Paradentitruncus

Paraechinorhynchus

Paragorgorhynchus

Paralongicollum

Paralueheia

Paraprosthenorchis.

Pararaosentis

Pararhadinorhynchus

Paratenuisentis

Parazhijinites

Paulisentis

Pilum

Plagiorhynchus

Polyacanthorhynchus

Polymorphus

Pomphorhynchus

Porrorchis

Profilicollis
287

290

281

276

277

281

290

.. 293

277

284

277

282

282

277

.. 294

274

294

278

279

279

281

.. 283

.. 290

284

.. 287

282

.. 290

.. 288

293

278

.. 280

.. 284

.. 282

.. 298

.. 282

.. 286

.. 293

.. 298

297

288

294

297
Promoniliformis ................................................................................................... 277

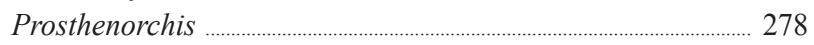

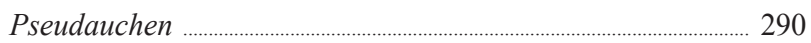

Pseudoacanthocephalus ............................................................................. 286

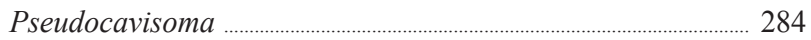

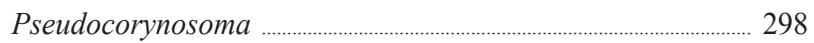

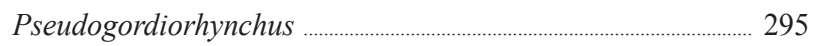

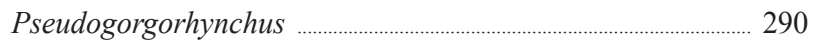

Pseudoleptorhynchoides .......................................................................... 290

Pseudolueheia _................................................................................. 295

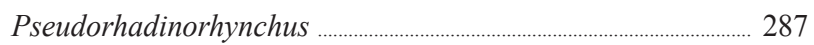

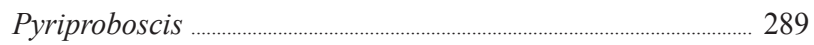

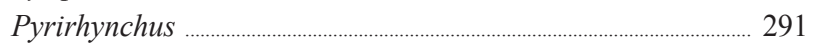

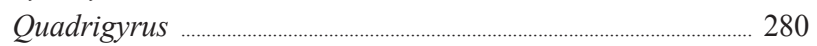

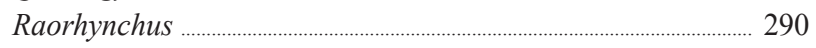

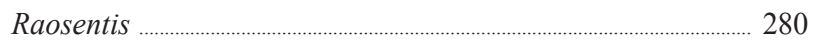

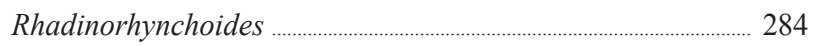

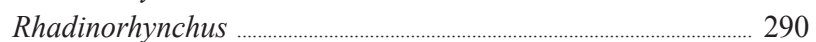

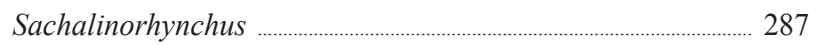

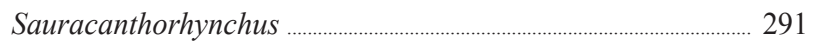

Sclerocollum .................................................................................................... 290

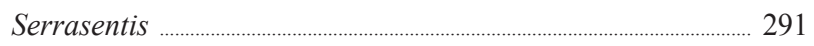

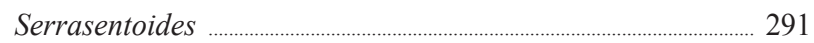

Slendrorhynchus ...................................................................................... 291

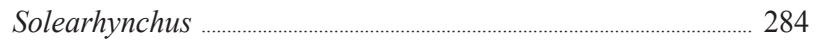

Southwellina ...................................................................................................... 298

Sphaerechinorhynchus ................................................................... 295

Sphaerirostris .................................................................................................... 293

Spiracanthus ......................................................................................................... 284

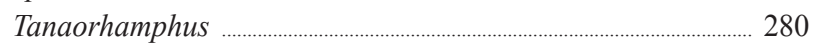

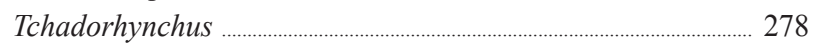

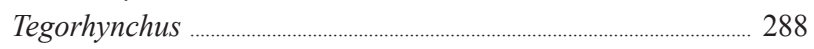

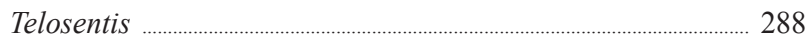

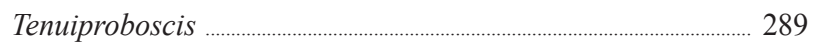

Tenuisentis .............................................................................................................. 283

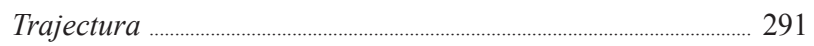

Transvena ........................................................................................................ 291

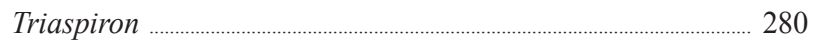

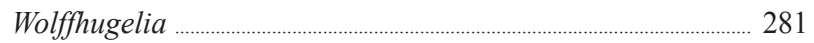

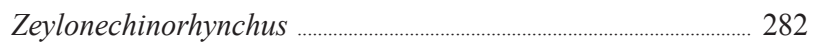

Zhijinites ............................................................................................................. 298 